University of Louisville ThinkIR: The University of Louisville's Institutional Repository

Electronic Theses and Dissertations

$12-2015$

\title{
Cardiovascular regulation and effects of respiratory motor training in patients with chronic spinal cord injury.
}

Bonnie Legg Ditterline

University of Louisville

Follow this and additional works at: https://ir.library.louisville.edu/etd

Part of the Circulatory and Respiratory Physiology Commons, Neurosciences Commons, Other Physiology Commons, and the Physiological Processes Commons

\section{Recommended Citation}

Ditterline, Bonnie Legg, "Cardiovascular regulation and effects of respiratory motor training in patients with chronic spinal cord injury." (2015). Electronic Theses and Dissertations. Paper 2299.

https://doi.org/10.18297/etd/2299

This Doctoral Dissertation is brought to you for free and open access by ThinkIR: The University of Louisville's Institutional Repository. It has been accepted for inclusion in Electronic Theses and Dissertations by an authorized administrator of ThinkIR: The University of Louisville's Institutional Repository. This title appears here courtesy of the author, who has retained all other copyrights. For more information, please contact thinkir@louisville.edu. 


\title{
CARDIOVASCULAR REGULATION AND EFFECTS OF RESPIRATORY MOTOR TRAINING IN PATIENTS WITH CHRONIC SPINAL CORD INJURY
}

\author{
By \\ Bonnie Legg Ditterline, MS \\ B.S., University of Kentucky, 2008 \\ M.S., University of Louisville, 2010
}

\begin{abstract}
A Dissertation
Submitted to the Faculty of

the School of Medicine, University of Louisville

in Partial Fulfillment of the Requirements

for the Degree of

Doctor of Philosophy

in Physiology and Biophysics

Department of Physiology and Biophysics

University of Louisville

Louisville, KY
\end{abstract}

December 2015 

CARDIOVASCULAR REGULATION AND EFFECTS OF RESPIRATORY MOTOR TRAINING IN PATIENTS WITH CHRONIC SPINAL CORD INJURY

\author{
By \\ Bonnie Legg Ditterline, MS \\ B.S., University of Kentucky, 2008 \\ M.S., University of Louisville, 2010
}

A Dissertation Approved on

2 December, 2015

by the following Dissertation Committee:

Alexander Ovechkin, MD., PhD

Dale Schuschke, PhD

Irving Joshua, $\mathrm{PhD}$

Claudio Maldonado, PhD

Ann Swank, PhD 


\section{DEDICATION}

This dissertation is dedicated to my grandfather, Sherwood Fifield Legg:

the one who first planted the seed 


\section{ACKNOWLEDGEMENTS}

I would like to sincerely thank my mentor, Dr. Alexander Ovechkin, for his

guidance and eternal patience: I have learned so much, and for that I am forever grateful. I would also like to thank my husband and kids, for the sacrifices they have made to make this goal possible; without them behind me, I wouldn't have made it this far. Additional gratitude is owed to my husband, alone, who provided countless hours of statistical assistance without complaint. I am also grateful for the support given to me by Sevda Aslan, who helped me shape ideas into methodologies, and guided me when I struggled. I also owe gratitude to my lab members, Eddie, Manpreet, and Goutam, who helped me gather the staggering amount of data contained herein. Thanks are also due to my committee members, who were always available to meet when I had questions or problems. Many thanks to my parents, who always supported me and taught me to keep moving forward. Finally, many thanks to my friends, Jess, Carrell, Christen, and Brittney, who were always there to listen when I felt like the stress was too much to bear alone. 


\begin{abstract}
CARDIOVASCULAR REGULATION AND EFFECTS OF RESPIRATORY MOTOR TRAINING IN PATIENTS WITH CHRONIC SPINAL CORD INJURY
\end{abstract}

Bonnie Legg Ditterline, M.S.

2 December 2015

This dissertation attempts to discover the mechanisms between cardiovascular and respiratory motor control post spinal cord injury (SCI): in normal, non-injured $(\mathrm{NI})$ persons, cardiovascular regulation is dependent upon respiration, but there is nothing that suggests the mechanism for this relationship post-SCI. Thus we hoped to evaluate various aspects of cardiovascular regulation to further illustrate how this relationship is changed or unchanged by $\mathrm{SCl}$.

Chapter I describes the anatomy and physiology of the spine, respiratory system, and cardiovascular system in a NI person, and then describes how the function of these systems is changed by SCl. In addition, we describe therapies available to persons with $\mathrm{SCl}$ to treat or manage these dysfunctions. Briefly, cardiovascular function is dependent on respiration, and motor and sensory activity is partially mediated by the spine. Thus, $\mathrm{SCl}$ will impair respiratory and cardiovascular function because neurons from these respective systems 
originate within the spine. In addition to that, we hypothesize that $\mathrm{SCl}$ will further impair reflexes due to the dependence of cardiovascular regulation on respiration. This relationship has not been studied post-SCI, but it has great therapeutic potential.

Chapter II details our specific aims and respective hypotheses: we think that poor respiration will exacerbate cardiovascular dysfunction (one) such that level and completeness will not correlate to cardiovascular functional outcomes (two); if hypotheses one and two are correct, then we hypothesize that we will be able to improve cardiovascular regulation by improving respiratory motor control. In Chapter III, we describe the methods and protocol by which we will either confirm or refute our respective hypotheses, including pulmonary and cardiovascular assessments, and statistical methods. Chapters IV, V, VI, and VII include our results and subsequent discussions, including validation of a particular method (Chapter IV); confirmation of cardiovascular impairment secondary to poor respiratory motor control (Chapter V); subsequent independence of functional outcomes and level of injury (Chapter VI); and improvements to cardiovascular regulation following improved respiratory motor control (Chapter VII).

Finally, in Chapter VIII, we conclude that there is still dependence of cardiovascular function on respiration post-SCI, and describe the potential benefits that respiratory motor training can have on this population. In addition, 
we detail future directions as we pursue our goal of developing respiratory motor training into a standard of care in persons with $\mathrm{SCl}$. 


\section{TABLE OF CONTENTS}

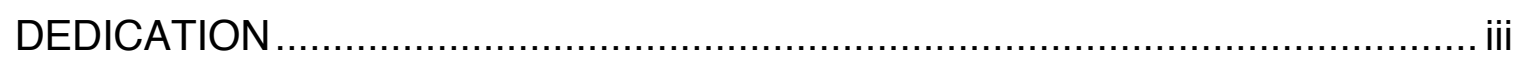

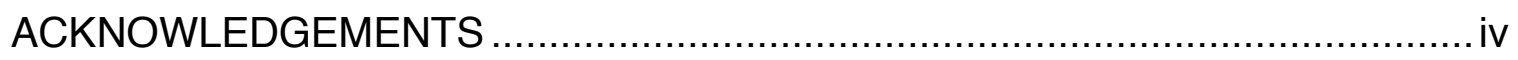

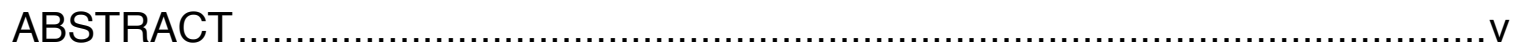

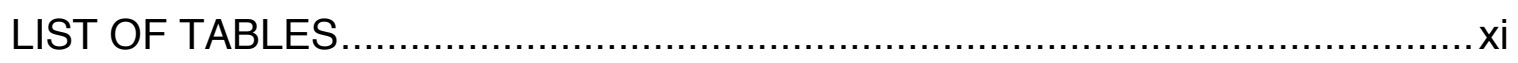

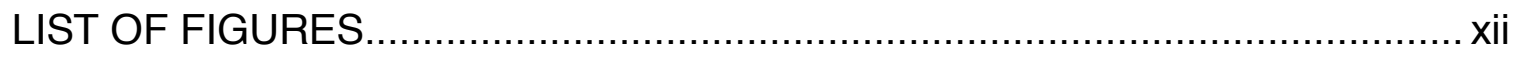

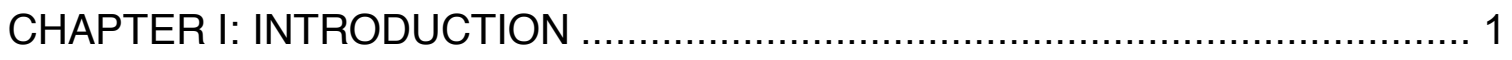

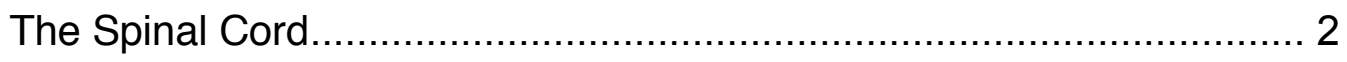

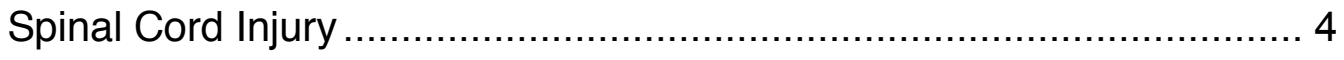

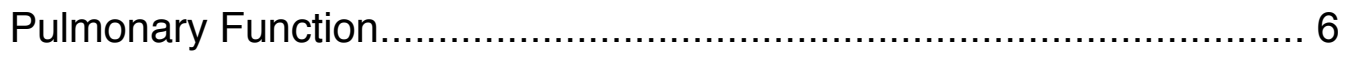

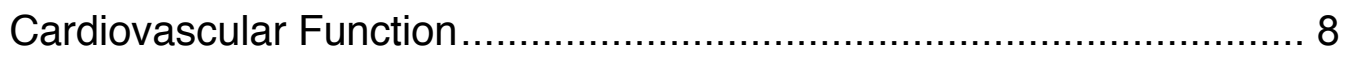

Pulmonary and Cardiovascular Function after Spinal Cord Injury ........ 16

Management of SCl-Induced Cardiovascular and Pulmonary

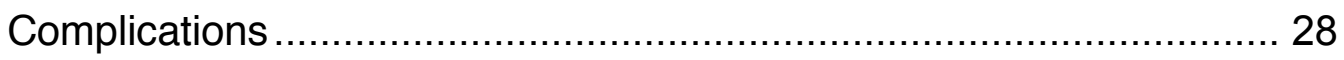

CHAPTER II: HYPOTHESES AND SPECIFIC AIMS .................................. 32

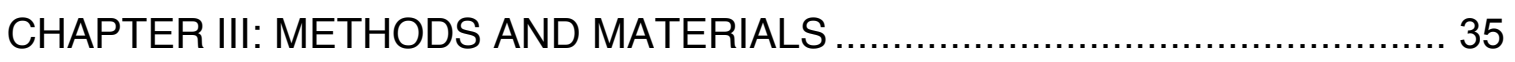

Respiratory Motor Control Assessment.......................................... 42

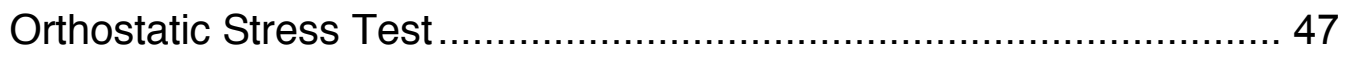

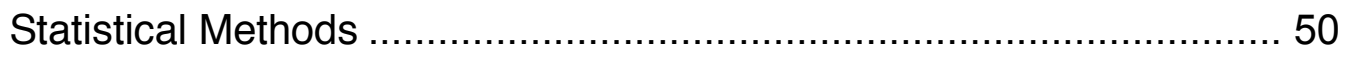




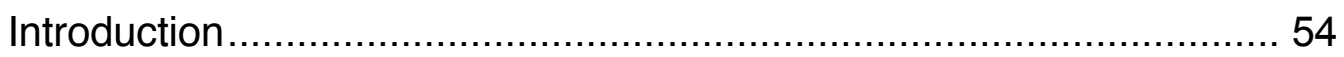

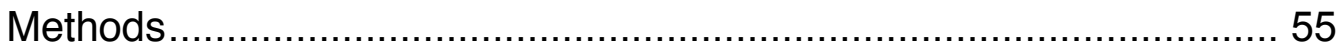

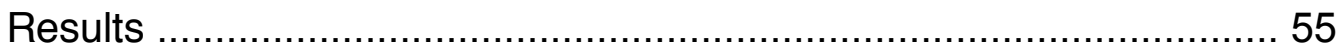

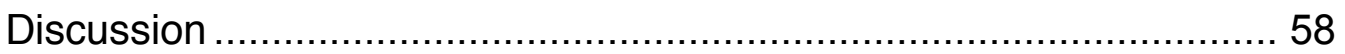

CHAPTER V: MECHANISMS OF RESPIRATORY AND CARDIOVASCULAR

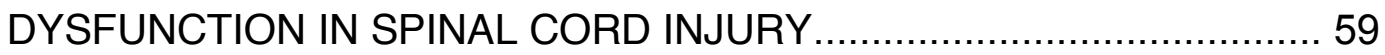

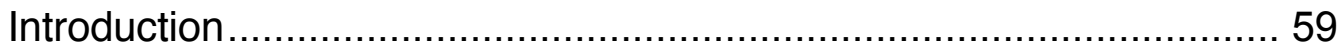

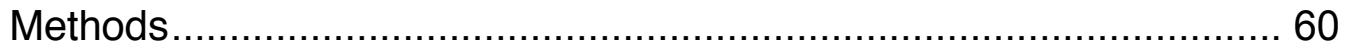

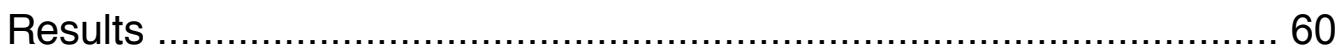

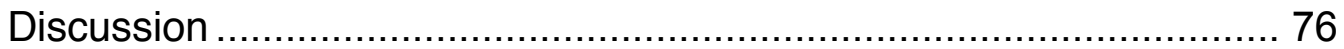

CHAPTER VI: CARDIOVASCULAR REGULATION AND LEVEL OF SPINAL

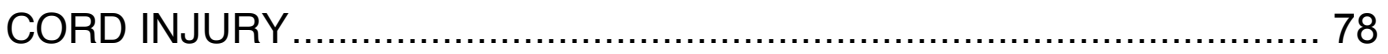

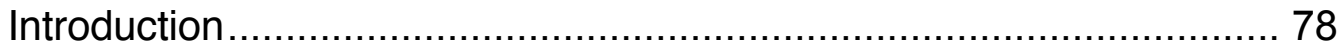

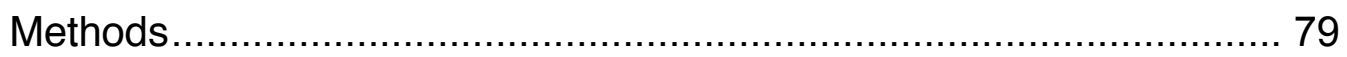

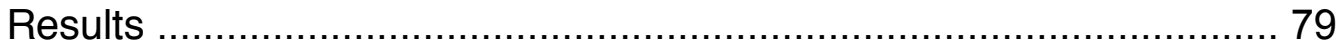

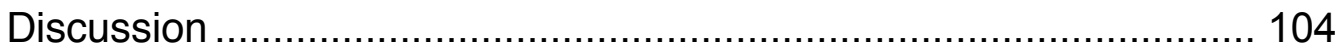

CHAPTER VII: CARDIOVASCULAR REGULATION POST RESPIRATORY

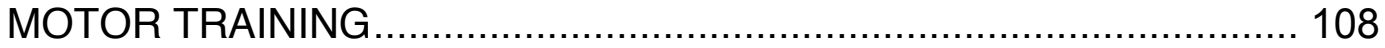

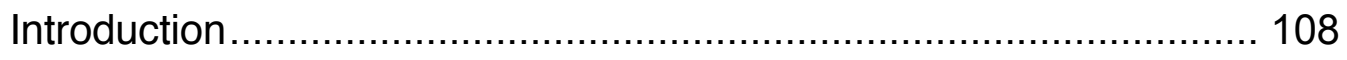

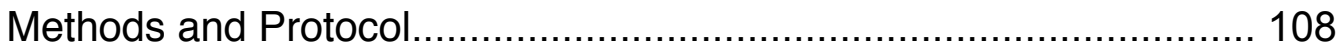

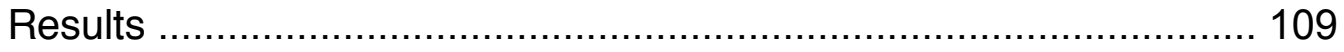




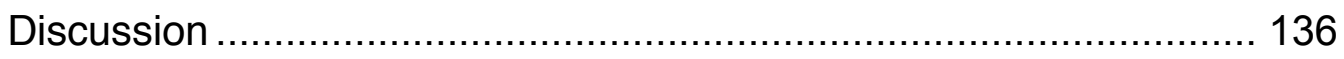

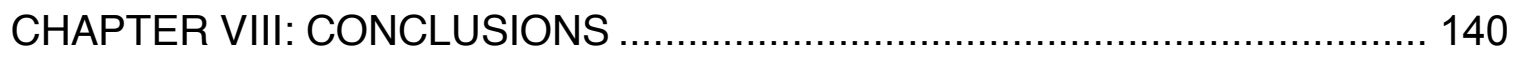

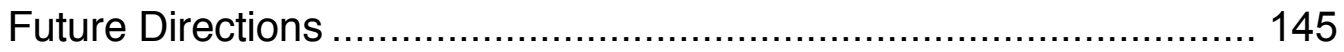

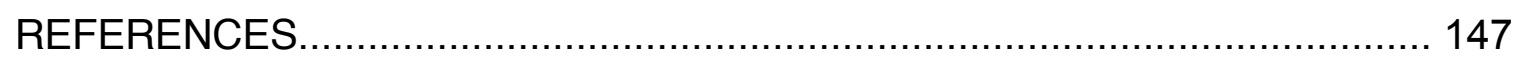

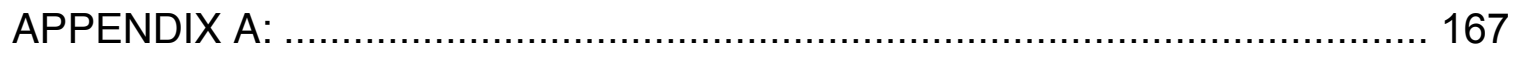

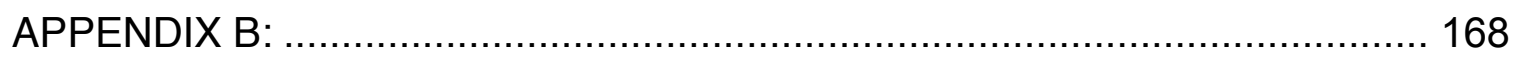

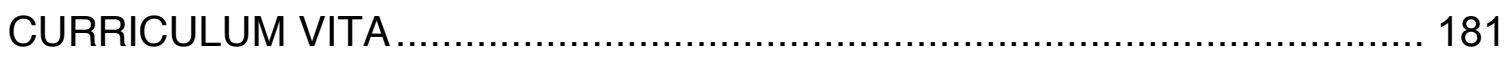




\section{LIST OF TABLES}

Table III-1: Demographics of Non-injured group ............................................ 38

Table III-2: Demographics of Respiratory Motor Training Group ....................... 39

Table III-3: Demographics of Control Group ............................................... 40

Table III-4: Univariate and Multiple Linear Regression Models Comparing Injured

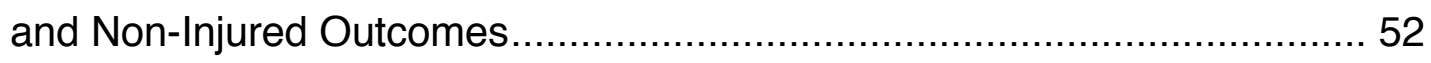

Table III-5: Univariate and Multiple Linear Regression Models Comparing RMT

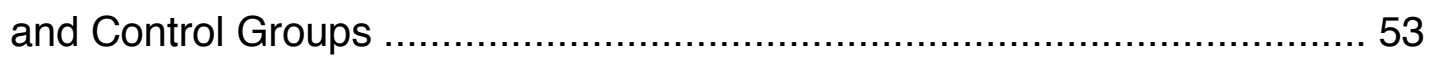

Table IV-1: Mean Outcomes of Valsalva Maneuver .......................................... 56

Table IV-2: ANOVA Results Comparing Valsalva Maneuver to Sustained, Maximum Expiratory Pressure Maneuver.............................................. 57 


\section{LIST OF FIGURES}

Figure V-1: Pulmonary Function Outcomes in SCl and NI Persons ................. 62

Figure V-2: Sympathetic Heart Rate Variability in SCI and NI Persons ............. 64

Figure V-3: Parasympathetic Heart Rate Variability in SCl and NI Persons ....... 65

Figure V-4: Change in RRI from Supine to Seated in SCI and NI Persons......... 66

Figure V-5 Change in SBP from Supine to Seated in SCl and NI Persons........ 67

Figure V-6: Change in DBP from Supine to Seated in SCI and NI Persons ....... 68

Figure V-7: Significant Up-up BRS Sequences in SCI and NI Persons ............. 69

Figure V-8 Up-up BEI in SCl and NI Persons ................................................ 70

Figure V-9 Significant Down-down BRS Sequences in SCI and NI Persons ...... 71

Figure V-10 Down-down BEI in SCI and NI Persons ..................................... 72

Figure V-11: BRS Slope During 5s MEP in SCI and NI Persons ..................... 74

Figure V-12: BRS Slope Following 5s MEP in SCI and NI Persons ................. 75

Figure VI-1: Pulmonary Function Outcomes by LOI ...................................... 82

Figure VI-2: Sympathetic Heart Rate Variability Outcomes by LOI.................... 83

Figure VI-3: Parasympathetic Heart Rate Variability Outcomes by LOI ............. 85

Figure VI-4: Change in RR Interval from Supine to Seated by LOI................... 87

Figure VI-5: Change in SBP from Supine to Seated by LOI ............................. 89

Figure VI-6: Change in DBP from Supine to Seated by LOI ............................ 91 
Figure VI-7: Significant Up-up BRS Sequences by LOI ..... 93

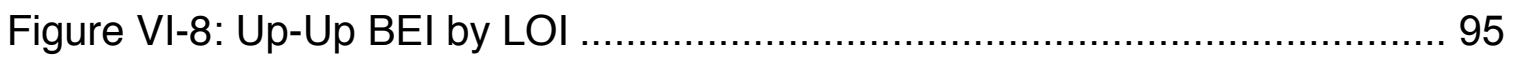

Figure VI-9: Significant Down-down BRS Sequences by LOI.......................... 97

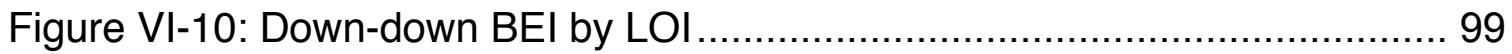

Figure VI-11: BRS Slope During 5s MEP by LOI ....................................... 101

Figure VI-12: BRS Slope Following 5s MEP by LOI ................................... 103

Figure VII-1: Pulmonary Function Outcomes Pre- and Post-RMT ................... 111

Figure VII-2: Sympathetic HRV Pre- and Post-RMT ................................... 113

Figure VII-3: Parasympathetic HRV Pre- and Post-RMT ............................... 115

Figure VII-4: Change in RR Interval During Orthostasis Pre- and Post-RMT ... 117

Figure VII-5: Change in SBP During Orthostasis Pre- and Post-RMT .............. 118

Figure VII-6: Change in DBP During Orthostasis Pre- and Post-RMT ............. 119

Figure VII-8 Significant Up-up BRS Sequences Pre- and Post-RMT ................ 122

Figure VII-9: Up-up BEI Pre- and Post-RMT ............................................... 124

Figure VII-10: Down-down BRS Sequence Slopes Pre- and Post-RMT .......... 126

Figure VII-11: Significant Down-down BRS Sequences Pre- and Post-RMT ... 128

Figure VII-12:Down-down BEI Pre- and Post-RMT .................................... 130

Figure VII-13: Change in BRS During 5s MEP Pre- and Post-RMT ................ 132

Figure VII-14: Change in BRS Following 5s MEP Pre- and Post-RMT ............ 134

Figure VIII-1: Continuous Hemodynamic Recording during Respiratory Motor

Training 


\section{CHAPTER I:}

\section{INTRODUCTION}

Spinal cord injury (SCl) can have devastating consequences to motor and autonomic regulation leading to the development of respiratory and cardiovascular diseases. These diseases progress at an accelerated rate compared to the non-injured $(\mathrm{NI})$ population and are also frequent co-morbidities. This is because, physiologically, the cardiovascular and pulmonary systems are closely related: respiration affects blood pressure (BP) and heart rate (HR). At the same time, cardiovascular parameters, blood concentration of $\mathrm{O}_{2}$ and $\mathrm{CO}_{2}$, and other factors, will alter respiration.

This close relationship means that improvements to one might improve the other: there is evidence that respiratory-cardiovascular relationships still exist following SCl. However, our understanding of the effects of $\mathrm{SCl}$ on cardiovascular-pulmonary interactions, and particularly on BP regulation, is decidedly incomplete. In addition to other researchers, we have found that persons with $\mathrm{SCl}$ who have better pulmonary function often have better cardiovascular function, but literature on more complex interactions does not exist. Perhaps because of this, cardiovascular and pulmonary dysfunctions in 
$\mathrm{SCl}$ are managed independently: pulmonary complications can be treated and ameliorated or improved in $\mathrm{SCl}$, but they are not evaluated for effects on cardiovascular function. There is therefore a clear gap in therapies available to improve pulmonary and cardiovascular function simultaneously. The most promising therapeutic candidate is respiratory training, which aims to improve pulmonary function by increasing respiratory motor performance. Since there is evidence the link between cardiovascular and pulmonary systems exists in $\mathrm{SCl}$, respiratory training that improves pulmonary function could likely lead to cardiovascular improvements. This intervention has never been used to improve $\mathrm{BP}$ regulation or to investigate the physiological relationships between pulmonary and cardiovascular function in $\mathrm{SCl}$ population. The objective of this study is therefore to investigate these mechanisms and to evaluate the therapeutic effects of resistive respiratory training as an important step toward our long-term goal of developing an evidence-based rehabilitation strategy for patients with SCI.

\section{THE SPINAL CORD}

The spinal cord, part of the central nervous system (CNS) along with the brain, is a bundle of nervous tissue and supporting cells enclosed within the vertebral column. The interior of the spinal cord is made up of neuron cell bodies, called the grey matter, surrounded by white matter comprised of myelinated ascending and descending axons (Crossman \& Neary 2010). This spatial arrangement allows the spinal cord to rapidly mediate information between the 
peripheral nervous system (neurons whose cell bodies reside outside the cord) (Boron \& Boulpaep 2009) and the cortex: sensory afferents receive information about the periphery from receptors and ascend rostrally through white matter to the cortex; CNS efferents, in turn, originate in the cortex and descend to synapse upon cell bodies within the grey matter in order to effect an appropriate response (Hall 2011). In addition to cortical reflexes, the spinal cord is the origin of many short-loop reflexes (involving an afferent sensory neuron and an efferent motor neuron) that bypass the cortex and thus respond more rapidly to stimuli.

While peripheral nervous system activity is largely controlled by the CNS, the autonomic nervous system (ANS - Greek for "self-governing") works independently: very little conscious control can change or interrupt the function of the ANS. The ANS controls visceral activity and is composed of two synergistic parts: the sympathetic nervous system (SNS) and the parasympathetic nervous system (PNS) (Boron \& Boulpaep 2009). The activity of the SNS and PNS are complementary and synergistic to maintain homeostasis: what the SNS excites, the PNS often inhibits. Sympathetic fiber chains consist of two neurons: a short presynaptic (preganglionic) neuron and a long postsynaptic (postganglionic) neuron. The presynaptic cell body resides in the gray matter and is excited or inhibited by supraspinal neurons; the postsynaptic cell body resides in the paravertebral ganglia. The SNS prepares the body for activity, and as such is responsible for increasing HR and vasomotor tone, among others. Sympathetic control of the heart emerges from the first five thoracic vertebrae, while vessel 
control emerges throughout the thoracic up to the first lumbar vertebrae. PNS presynaptic neurons emerge from the brainstem (Cranial nerves, or CN, III, VII, IX, and X) and sacral spine and synapse on postsynaptic neurons embedded within, or in close proximity to, targeted tissues. The PNS controls the body during rest and sedentary activity and would thus decrease HR and inhibit vasoconstriction (Hall 2011).

\section{SPINAL CORD INJURY}

The spinal cord is an essential component of the CNS through which all motor and peripheral sensory information travel. By coordinating and relaying information from the PNS and ANS, as well as utilizing short-loop reflexes that originate therein, the spinal cord enables the CNS to quickly adapt the internal to the external environment and maintain homeostasis. Because of this complexity and reliance on the spinal cord, it is evident why a small spinal injury can still impair or eliminate entirely communication between the CNS, PNS or SNS and their target organs or tissues. Indeed, a small lesion to the spinal cord, a size that would be inconsequential to cortical function, could cause widespread dysfunction and death (Haines 2006).

It is estimated that there are as many as $1,275,000$ persons in the United States live with a traumatic SCl. The most common cause of SCl was workrelated accidents ( 28 percent) followed by motor vehicle accidents ( 24 percent) (Anthony Cahill et al 2009, Fox et al 2015). A traumatic SCl is any injury to the 
spinal cord not caused by disease. The severity of complications post-injury depend on the location in which the injury occurs and the number of pathways spared. Animal models of SCI have found that, within 30 minutes post-injury, the affected neurons undergo axonal degeneration. Axons distal to the injury are broken into fragments, while axons proximal to the injury recede; ultimately the distance between the proximal and distal axons increases and the efferent targets of this tract are disconnected from the cortex (Conforti et al 2014, Kerschensteiner et al 2005). Human postmortem analysis has found significant correlation to injured tracts and functional deficits (Cohen-Adad et al 2011, Steward et al 2003). Function rostral to the lesion is therefore neurologically intact, while reflexes and pathways caudal to the lesion function without supraspinal control (Maier \& Schwab 2006).

Injury is classified by the system developed by the American Spinal Injury Association (ASIA), which describes the severity and location of the injury based on the number of active limb muscles and preserved sensory dermatomes below a spinal segment. Injuries are therefore identified by their neurological level of injury (i.e., C7) and the ASIA Impairment Score (AIS), and not necessarily the physical location upon the spinal cord at which the injury occurs. Injury severity is grouped from $A$ to $E$, where $A$ is the most severe injury, with neither sensory nor motor function below the level of injury, and $E$ has no detectible deficits (Committee et al 2012). 
Despite recent improvements to health care and overall increases in life expectancy, a person with a SCI still has an increased mortality rate when compared to age-matched counterparts, a rate that has remained unchanged since the 1980s (NSCISC 2014). A 20-year old, non-injured person has a life expectancy of about 60 years; a 20-year old person with a $\mathrm{SCl}$ has an average life expectancy ranging from 19 years (upper cervical, ventilator-dependent lesion) to 53 years (incomplete lesion at any level). Leading causes of death in the $\mathrm{SCl}$ population are similar to the non-injured population (respiratory diseases, and heart and vascular diseases) but these diseases are exacerbated by SCl. An injured person, therefore, is more likely to develop these diseases than a noninjured person and they are more likely to die from them (Krause \& Saunders 2011, NSCISC 2014).

\section{PULMONARY FUNCTION}

Cellular metabolism requires oxygen (in the form of $\mathrm{O}_{2}$ ) and creates carbon dioxide $\left(\mathrm{CO}_{2}\right)$ in the process; ventilation is therefore a life-sustaining process because all cells must be continuously supplied with $\mathrm{O}_{2}$ and removed of $\mathrm{CO}_{2}$. The automatic respiratory rhythm is generated in the central pattern generator, located on the ventral medulla, largely within the Pre-Bötzinger Complex (Lumb \& Nunn 2005). Oscillating action potentials within the PreBötzinger Complex excite premotor neurons that travel through the spinal cord to synapse on pools of respiratory motor neurons (Forster Hv 2004, Smith 1991). 
Motor neurons initiate inspiration by contracting the diaphragm, intercostal, and accessory muscles of inspiration, expanding the thoracic cavity and filling alveoli with atmospheric air. The phrenic nerve emerges from the third through fifth cervical vertebrae to innervate the diaphragm (Routal \& Pal 1999). The diaphragm descends caudally into the abdominal cavity, increasing the rostralcaudal volume of the intrathoracic cavity and the lateral diameter of the lower ribcage (Lumb \& Nunn 2005). The intercostal muscle fibers exist in the space between each rib. During inspiration, motor neurons preferentially contract the rostral and dorsal external intercostals and the parasternal internal intercostals. This selective activation increases the rostral-caudal length of the ribcage by pulling each rib rostrally, as well as increasing the anterior-posterior diameter of the ribcage by lifting the sternum away from the spine (the diameter can be 20 percent greater during a maximal inspiration than rest) (Boron \& Boulpaep 2009, Hall 2011, Lumb \& Nunn 2005). The accessory muscles of inspiration assist the upper intercostals by lifting the sternum and first two ribs (sternocleidomastoid and scalene muscles, respectively) (Hall 2011). The lungs are coupled to the rib cage due to the pleura that exist between them and relative vacuum created therein: an injury to the pleura that eliminates this vacuum will cause the lungs to collapse and the ribs to expand outward. Therefore, when ribs expand the volume of the thoracic cavity during inspiration, the lung tissue expands passively. Atmospheric air flows down its pressure gradient into the lungs, ending 
at the alveoli where $\mathrm{O}_{2}$ can then diffuse into the blood (Boron \& Boulpaep 2009, Hall 2011).

Passive expiration requires no muscle activity; instead, inspiratory muscles relax, the diaphragm ascends rostrally, the rib cage shrinks, and both press upon the lungs. Voluntary expiration (e.g., a controlled expiration for speech) or an involuntary expiration (e.g., a cough) inhibits inspiratory muscles via the medulla, thus interrupting the eupnic rhythm, and simultaneously contract the abdominal, oblique, intercostal and pectoralis muscles to decrease the volume of the intrathoracic cavity (Boron \& Boulpaep 2009, Hall 2011).

The eupnic rhythm and subsequent movement of the thoracic cavity is what helps support cardiovascular function: every inspiration decreases intrathoracic pressure, which increases venous return to the right atrium, while the increased intrathoracic pressure during expiration will decrease venous return. The lungs therefore create the respiratory pump, which plays a large role in normal cardiovascular function (Vincent et al 2011).

\section{CARDIOVASCULAR FUNCTION}

The cardiovascular system evolved as a means to provide organs and tissues with inspired $\mathrm{O}_{2}$ from the lungs. Single-celled and incomplex organisms can meet their metabolic needs and eliminate cellular waste by simple diffusion alone; as organism complexity increases, however, simple diffusion is not enough because diffusion efficiency decreases exponentially as distance 
increases. To overcome this limitation, the human body evolved not only a medium into which oxygen can diffuse (blood), but vessels to deliver oxygen to the tissues (the vasculature), and a pump to ensure that oxygen and metabolic wastes are continuously cycled (the heart) (Boron \& Boulpaep 2009). This evolution has created a complex interdependence between the lungs and cardiovascular system in order to optimize $\mathrm{O}_{2}$ delivery to the tissues.

The heart is the pump that delivers $\mathrm{O}_{2}$ to the organs. The sinoatrial (SA) node is the primary pacemaker and generates an intrinsic rhythm of around 100 heartbeats per minute (BPM); under normal circumstances, all cardiac electrical activity originates therein. The inherent rhythmicity of the SA node is due to the time- and voltage-dependent depolarization of three voltage-gated channels, the sum of which determines the time between action potentials and thus controls the frequency of action potentials that propagate from the SA node. (Berne 2010, Boron \& Boulpaep 2009, Hall 2011). From the SA node, the action potentials propagate through gap junctions in atrial myocytes and through the intermodal pathways to arrive at the atrioventricular (AV) node. Action potentials in the AV node propagate to the bundle if His, which splits into the right and left bundle branches and terminates at the Purkinje fibers, leading to ventricular contraction (Berne 2010, Boron \& Boulpaep 2009, Hall 2011).

In the pulmonary capillaries, blood is removed of $\mathrm{CO}_{2}$ and saturated with $\mathrm{O}_{2}$ in order to be delivered to the tissues. The delivery of $\mathrm{O}_{2}$ begins with the aorta, which branches until it ends with the capillaries. In the capillaries, $\mathrm{O}_{2}$ 
diffuses into the tissues and $\mathrm{CO}_{2}$ diffuses into the plasma. The blood leaves the capillaries where it is collected by the venous system, which is then circulated to the right side of the heart. The aorta (an artery) branches into increasingly narrower vessels until the capillary networks are formed. Collectively, the vessels from the aorta up to the smallest arterioles are called the high-pressure system (Boron \& Boulpaep 2009). The walls of the high-pressure system are made of three layers: the intima (closest to the interior of the vessel), the media, and the adventitia. The intima layer is a continuous layer of endothelial cells adjacent to organized networks of collagen, fibronectin, and laminin, which provide stability and strengthen the vessels against the high pressures within. Surrounding this connective tissue is the layer of smooth muscle, the media layer, which determines the diameter of the vessels. Finally, the adventitia lies outside the smooth muscle and contains nerve terminals that regulate the media (Cronenwett \& Johnston 2014).

Arterioles are the primary regulators of blood flow through the vasculature, and thus have the greatest effect on BP. When an artery is dilated, the diameter of the artery increases because circularly-oriented smooth muscle in the media layer is relaxed. The volume of blood within them increases, increasing the flow of blood to the tissues and decreasing the BP within them (Cronenwett \& Johnston 2014, Spronck et al 2014). Plasma from the capillaries drains into the low-pressure system: the venous side of the circulation. The anatomy of the veins is similar to arteries: there are tunica, media, and adventitial layers; 
however, the veins are less structured and more compliant than the arteries due to their composition. The adventitial layers contain nerve endings to influence vessel capacitance and vasomotor tone. The veins also have increased collagen, decreased elastin and less smooth muscle, which allows the veins to stretch and accommodate increases in blood volume with little change in pressure (Boron \& Boulpaep 2009, Cronenwett \& Johnston 2014, Hall 2011).

\section{Cardiovascular Regulation}

In addition to the mechanical relationship (i.e., the respiratory pump), there are complex reflexes that exist between the lungs and cardiovascular system in order to match ventilation with perfusion and ensure a stable supply of $\mathrm{O}_{2}$ to the tissues. To do this, BP is maintained within a narrow range by the ANS and reflex arcs that augment $\mathrm{HR}$ and vasomotor tone in response to changes in BP.

The cardiac rhythm generated by the SA node is mediated by both PNS and SNS. The SNS exerts inotropic and chronotropic effects over the heart via the cardiac nerves. Norepinephrine or epinephrine released into the SA node will increase the activity of inward channels, allowing the SA node to reach threshold more quickly, thus increasing $\mathrm{HR}$ and decreasing time between R-R intervals (RRI). Catecholamines also act as a positive inotrope on ventricular muscle by increasing available $\mathrm{Ca}^{2+}$ to increase the force of contraction (Boron \& Boulpaep 2009). The PNS exerts control over the HR via CN X, or the vagus nerve. Acetylcholine is released onto the SA node which slows inward currents and 
increases outward currents, lengthening RRI by slowing the conduction velocity through atrial myocytes (Lewis et al 2001).

Regulation of BP within the vasculature is regulated neurally and locally. Locally, increased BP increases shear stress upon the vessel wall, releasing Nitric Oxide (NO) from the endothelial cells. Nitric Oxide rapidly initiates relaxation locally as well as triggering a secondary release of NO from upstream vessels (Cronenwett \& Johnston 2014, Hall 2011).

Autonomically, the PNS and SNS exert complementary control over the vasculature. However, tonic control of the vasculature is primarily due to the activity of the SNS; the PNS largely controls the vasculature during reflex responses (Hall 2011). Sympathetic control, originating in the medulla, fires continuously to maintain vasomotor tone (a state in which the blood vessels are partially contracted). This increases peripheral resistance within the arteries and decreases venous capacitance, increasing the amount of blood that returns to the right atrium. Inhibition of tonic vasomotor activity generated by the SNS, either at the level of the medulla or spinal cord, causes drastic drops in BP due to widespread vasodilation; conversely, large increases in vagal activity have little effect on vasomotor tone (Cronenwett \& Johnston 2014, Hall 2011).

Mechanical oscillations in BP engage high-pressure baroreceptors located in the adventitia of the carotid sinus and the aortic arch (Persson \& Kirchheim 1991). Distension of the vessels send an increasing frequency of action potentials to the Nucleus Tractus Solitarii (NTS) via CN IX, signaling an increase 
in mean arterial BP; alternatively, a decrease in BP decreases receptor activity until it stops altogether around 40 to $60 \mathrm{mmHg}$. Firing frequency is further increased by rapid increases in BP: twice as many action potentials are generated by an increasing pressure of $150 \mathrm{mmHg}$ than a stationary pressure of $150 \mathrm{mmHg}$ (Hall 2011, Persson \& Kirchheim 1991, Scher et al 1991). The NTS responds to an increase in BP by inhibiting descending sympathetic efferents and exciting vagal efferents, causing both a decrease in HR and arterial vasoconstriction. A decreased BP removes descending sympathetic inhibition while inhibiting vagal efferents in order to increase HR and increase arterial vasomotor tone (Berne 2010, Persson \& Kirchheim 1991). Baroreflex response of the capacitance vessels is minimal, with the exception of orthostatic stress: a rapid change to an upright posture can cause blood to pool in the viscera and legs, which increases sympathetic activity to the abdominal and deep veins in order to assist venous return (Persson \& Kirchheim 1991).

Within the pulmonary arteries and walls of the atria are low-pressure stretch receptors. These receptors balance the tonic, sympathetic excitation from the high-pressure receptors by tonically inhibiting descending sympathetic visceral and leg vessels (Burgh Daly 1997). However, low-pressure receptors do not detect systemic BP, but instead blood volume. Increased venous return increases the amount of blood in the right atrium and pulmonary arteries; the subsequent vessel and atria stretch stimulates vagal afferents to inhibit descending sympathetic vasomotor centers (Persson \& Kirchheim 1991, Sala- 
Mercado et al 2014). A rapid change in blood volume is thus detected and buffered into the circulation by changes in vascular tone in order to maintain a stable BP. For example, due to the activity of these receptors, an increased blood volume within the right atrium of 300 milliiters will only raise peripheral BP by 15 $\mathrm{mmHg}$ or less; denervation of these receptors, however, would cause BP to rise by $100 \mathrm{mmHg}$ or more (Hall 2011).

Within the lungs are sensory afferents that integrate pulmonary and cardiovascular control, including a primitive reflex called the "respiratory sinus arrhythmia" (RSA) (Burgh Daly 1997, Hayano \& Yasuma 2003). Afferent, slowly adapting mechanoreceptors (SARs) in the bronchi and bronchioles fire with increasing lung volume; these receptors ensure that the lungs do not over inflate during inspiration (called the Hering-Bruer reflex) (Hall 2011, Lumb \& Nunn 2005) and coordinate cardiovascular activity with respiratory rhythm (Lee \& Yu 2014). The increased pressure within the airways excites the SARs, which send impulses to the NTS via the vagus nerve. Rhythmic impulses and impulses below lung volume threshold ( $20 \mathrm{mmHg}$ or less) elicit tachycardia by vagal inhibition, while inflation above the threshold $(25 \mathrm{mmHg}$ and above) elicits bradycardia (Lee \& Yu 2014). In addition, vagal afferents also influence the activity of descending sympathetic vasomotor tone. Normal inspiration inhibits sympathetic vasomotor neurons with increasing frequency, so that as lung volume increases, vascular resistance decreases. In this manner, HR and vasodilation increase upon inspiration and decrease upon expiration. Afferent, slowly adapting 
mechanoreceptors (SARs) in the bronchi and bronchioles fire with increasing lung volume; these receptors ensure that the lungs do not over inflate during inspiration (called the Hering-Bruer reflex) (Hall 2011, Lumb \& Nunn 2005) and coordinate cardiovascular activity with respiratory rhythm (Lee \& Yu 2014). The increased pressure within the airways excites the SARs, which send impulses to the NTS via the vagus nerve. Rhythmic impulses and impulses below lung volume threshold ( $20 \mathrm{mmHg}$ or less) elicit tachycardia by vagal inhibition, while inflation above the threshold $(25 \mathrm{mmHg}$ and above) elicits bradycardia (Lee \& Yu 2014). In addition, vagal afferents also influence the activity of descending sympathetic vasomotor tone. Normal inspiration inhibits sympathetic vasomotor neurons with increasing frequency, so that as lung volume increases, vascular resistance decreases. In this manner, HR and vasodilation increase upon inspiration and decrease upon expiration (Burgh Daly 1997, Lee \& Yu 2014).

Finally, there are also chemoreceptors that detect $\mathrm{O}_{2}$ saturation in the blood, directly or indirectly (via $\mathrm{CO}_{2}$ or $\mathrm{pH}$ ) that alter respiration, $\mathrm{HR}$, and vasoconstriction together. In the presence of hypoxia, afferents that travel to the medulla increase respiration while also inhibiting the cardioinhibitory center, which increases HR and vasoconstriction (Boron \& Boulpaep 2009, Hall 2011). The chemoreceptors will ultimately attenuate the influence of the RSA on the vasculature; the resulting hyperventilation, despite frequent changes in lung volume and thus more stimulation, will not cause vasodilation despite the increased afferent activity (Burgh Daly 1997). 


\section{PULMONARY AND CARDIOVASCULAR FUNCTION AFTER SPINAL CORD INJURY}

Despite the interdependence of the cardiovascular and pulmonary systems, the spinal cord is still an essential part of their normal function: all respiratory motor and sympathetic cardiovascular neurons originate therein. It is logical to assume that interdependence of cardiovascular and pulmonary interactions still exist despite $\mathrm{SCl}$, and the literature confirm this: acutely, morbidity and mortality rates are significantly decreased when cardiovascular and pulmonary deficits are addressed together (Casha \& Christie 2011, Walters et al 2013), and chronically, persons with greater pulmonary function outcomes demonstrate higher resting BPs (Frisbie 2005). However, despite the foundation of knowledge about normal cardiopulmonary function, little is known of the relationship between them post-SCl: cardiovascular and pulmonary dysfunction in SCl are largely studied and treated independently.

\section{Pulmonary function after spinal cord injury}

In persons with $\mathrm{SCl}$, respiratory motor function decreases as the lesion ascends rostrally due to the emergence of respiratory motor neurons throughout the cervical and thoracic segments. Consequently, pulmonary function correlates strongly with level of injury (Ovechkin et al 2010, Schilero et al 2009). An injury 
rostral to the emergence of the phrenic nerve (C3) can result in partial or total paralysis of all inspiratory and expiratory muscles, rendering a person ventilatordependent (Brown et al 2006). An injury at C7 would neurologically spare the diaphragm, the sternocleidomastoid and most of the scalene muscles, while impairing or eliminating altogether the function of the intercostal and abdominal muscles. An injury at T6 would spare intercostals rostral to the lesion, while impairing caudal intercostal and abdominal muscles. Paralysis, paresis, or spasticity of respiratory muscles would therefore impair not only normal breathing, but speech, forced expiration, and cough reflexes as well, because they require recruitment of additional respiratory muscles (Schilero et al 2009).

Provided the injury spares the diaphragm, persons with $\mathrm{SCl}$ typically have preserved eupnea because the diaphragm can compensate for impaired respiratory muscles (Fuller et al 2006, Schilero et al 2009). However, increased activity of the diaphragm and accessory muscles of inspiration in tetraplegia, coupled with increased compliance of the abdominal wall, causes "paradoxical breathing," where the diameter of the lower rib cage shrinks during inspiration. This leads to decreased tidal volume and an increased residual volume within the lungs, decreasing the amount of $\mathrm{O}_{2}$ available in the alveoli for diffusion.

Paradoxical breathing combined with a decreased $\mathrm{O}_{2}$ intake can ultimately lead to chronic respiratory muscle fatigue due to increased metabolic work of quiet breathing (De Troyer et al 1986, De Troyer \& Heilporn 1980, Estenne \& De Troyer 1986). In addition to impairments to normal breathing, respiratory efforts 
that require recruitment of additional inspiratory or forced expiratory muscles are also impaired. Cough, for example, requires recruitment of abdominal muscles in addition to external intercostals to increase intrathoracic pressure; persons with cervical and upper-thoracic SCI frequently have weak or ineffective cough due to inactivity of these muscle groups (Schilero et al 2009, Terson de Paleville et al 2011, Vinit \& Kastner 2009).

Decreased pulmonary and respiratory motor function in $\mathrm{SCl}$ are therefore significant predictors of future respiratory diseases (Stolzmann et al 2010). Decreased cough capacity, due to paralysis and paradoxical movement of the rib cage, limits the ability of a person to clear mucus or irritants from his or her airways; this traps mucus in the lower airways, increasing the risk of atelectasis and pulmonary infection (Estenne \& Gorini 1992, Fishburn et al 1990). Risk of future pulmonary disease increases as level of injury ascends rostrally (Cotton et al 2005, Tollefsen \& Fondenes 2012). Additionally, long-term pulmonary function in $\mathrm{SCl}$ is decreased, as normal age-related declines in ability and lung compliance are accelerated by injury (Stolzmann et al 2008). A person with SCI is therefore more likely to develop and succumb to respiratory diseases than an age-matched, non-injured counterpart (Garshick et al 2005, Middleton et al 2012). 
Evaluation of Pulmonary Function in individuals with Spinal Cord Injury

Respiratory motor function in $\mathrm{SCl}$ is frequently measured indirectly by assessing pulmonary function outcomes. Spirometric outcomes, like FVC and FEV1, measure (in Liters per second (L/s)) inspiratory and expiratory capacity as a function of time and can indicate respiratory muscle activation (Miller et al 2005), while maximal static airway pressure generation (in $\mathrm{cmH}_{2} \mathrm{O}$ ) indirectly measures respiratory muscle activity and ability (Schilero et al 2009, Stolzmann et al 2008). However, pulmonary function outcomes can only broadly indicate muscle impairment. Indeed, pulmonary function is additionally affected by completeness of injury, age, time since injury, and weight, all of which could confound an interpretation of respiratory muscle activity by this method (Mateus et al 2007). In order to illustrate individual respiratory muscle activity and pattern of activation, pulmonary function outcomes should therefore be combined with surface electromyography (Ovechkin et al 2010). Surface electrodes placed over respiratory muscles during $\mathrm{FVC}, \mathrm{FEV}_{1}$, and maximal inspiratory and expiratory airway pressure generations can illustrate deficits in particular muscles as well as the pattern and timing of activation during these maneuvers, an impossibility with pulmonary function outcomes alone (Aslan et al 2013, Ovechkin et al 2010).

$\mathrm{FVC}$ and $\mathrm{FEV}_{1}$ correlate significantly with level of injury due to the increasing dysfunction or inactivity of forced-expiratory respiratory muscles as the lesion ascends rostrally (Almenoff et al 1995, Linn et al 2000 ). Airway pressure generation correlates linearly with motor-complete injuries, but not motor- 
incomplete injuries (Mateus et al 2007). Provided the injury spares the diaphragm, maximum inspiratory pressure generation is usually greater than maximum expiratory pressure (MEP), which is opposite the non-injured population (Gounden 1997, Schilero et al 2009). Pulmonary function tests coupled with surface electromyography have further found that respiratory muscle patterns of activation correlate significantly with level of injury and pulmonary function. Finally, there is increased compensatory activity of muscles rostral to the lesion, which can exacerbate paradoxical breathing (Ovechkin et al 2010, Schilero et al 2009). It would logically follow that decreased respiratory motor function would then decrease cardiovascular function. The paradoxical breathing and poor muscle activity cause smaller changes in intrathoracic pressure with each breath, thus decreasing the strength of the respiratory pump and subsequent BP oscillations. The paradoxical breathing also creates poor ventilation at the base of the lungs, decreasing gas exchange and altering cardiovascular function due to activation of chemoreceptors. However, in $\mathrm{SCl}$, the relationship between cardiovascular and pulmonary function is only broadly correlated. The extent to which pulmonary dysfunction creates or exacerbates cardiovascular dysfunction in $\mathrm{SCl}$ is therefore unknown.

\section{Cardiovascular Function after Spinal Cord Injury}

Like pulmonary function, the degree of cardiovascular impairment in $\mathrm{SCl}$ is dependent upon the location and severity of the injury to the spinal cord. 
Descending tracts rostral to the lesion function normally, while tracts caudal to the lesion are isolated from supraspinal control; targets caudal to the lesion are therefore largely under vagal or peripheral reflex control (Teasell et al 2000). Meta-analyses of cardiovascular dysfunction in $\mathrm{SCl}$ found that cervical injuries frequently experience resting bradycardia and persistent hypotension when compared to able-bodied persons; upper thoracic (T6 and above) lesions experience hypotension when compared to able-bodied persons but not always bradycardia; and finally lower thoracic (below T6) and lumbar injuries have mean cardiovascular outcomes similar to able-bodied persons (Frankel \& Mathias 1992, West et al 2012). Postmortem histology affirms this relationship: persons with greater white matter degeneration of cardiovascular tracts caudal to the spinal cord lesion experienced greater cardiovascular dysfunction in life (Furlan et al 2003, Krassioukov 2006). Despite the overall decrease in sympathetic activity, resting autonomic tone in $\mathrm{SCl}$ is frequently normal because the body decreases vagal activity and increases sensitivity of peripheral a-adrenergic receptors in an attempt to restore balance to autonomic tone (Gondim et al 2004, Grimm et al 1997). Despite remodeling in an effort to maintain baseline BP, cardiovascular response to stress can be seriously impaired. Two of the most common dysfunctions experienced in $\mathrm{SCl}$ result from the lack of supraspinal sympathetic control: orthostatic hypotension and autonomic dysreflexia.

Orthostatic hypotension $(\mathrm{OH})$ is a drop on $\mathrm{BP}$ of $20 \mathrm{mmHg}$ systolic (SBP) or $10 \mathrm{mmHg}$ diastolic (DBP) upon assumption of an upright posture. Typically, a 
passive position change (i.e., without recruitment of skeletal muscle) causes blood to pool in the periphery and decreases venous return, which increases efferent SNS activity to bring BP back to baseline. Impairment of the SNS in SCI, coupled with paralysis of skeletal muscles, causes blood to pool within the deep veins of the legs and viscera, decreasing cerebral perfusion that can lead to dizziness, nausea, fatigue, or syncope (Furlan et al 2003, Phillips \& Krassioukov 2015, Popa et al 2010).

Some degree of $\mathrm{OH}$ is common in all levels of $\mathrm{SCl}$ : cervical injuries typically have significantly lower BP and $\mathrm{HR}$ values while upright when compared to able-bodied persons, and are more also likely to experience syncope (West et al 2012); upper thoracic injuries experience varying degrees of hypotension and bradycardia depending on the amount of sympathetic innervation of the heart and vasculature (West et al 2013, West et al 2012); and lower thoracic injuries can still demonstrate a blunted reflex response due to the paralysis of leg vasculature or desensitization of the reflexes due to inactivity (Wecht et al 2003).

Autonomic dysreflexia $(A D)$ is a reflex vasoconstriction of the blood vessels in the periphery that can affect up to 70 percent of persons with cervical or upper-thoracic SCI (Krassioukov et al 2007). Normally, visceral or cutaneous stimuli engage spinal reflexes that constrict the peripheral vasculature in order maintain homeostasis; the reflexes are inhibited by descending sympathetic neurons that originate in the brainstem or cortex. In a SCI at T5-6 or above, however, disconnection from the brainstem can cause the peripheral vasculature 
to constrict freely until the stimulus is identified and removed (Krassioukov et al 2007, McGillivray et al 2009). In particular, disconnection of the splanchnic vascular bed from supraspinal inhibition has been identified as a major component of $A D$ due to the large volume of blood stored therein (Krassioukov et al 2007, Previnaire et al 2012). Episodes of untreated AD thus can lead to BPs of $200 / 100 \mathrm{mmHg}$ or more, leading to seizures, coma, or death.

Post-SCl, the reflex arc below the level of injury also strengthens. The aadrenergic receptors in the periphery become hypersensitive to catecholamines, either due to increased sensitivity of the receptors themselves or decreased reuptake of synaptic norepinephrine (Arnold et al 1995, Teasell et al 2000). This not only increases activity of the sensory afferents, but rat models have demonstrated increased sensitivity of a-adrenergic receptors on vascular smooth muscle in the splanchnic and renal beds (Brock et al 2006, McLachlan \& Brock 2006). Responses are further strengthened post-injury due to remodeling within the spinal cord that not only increases the number of spinal interneurons that participate in these reflexes but the capacity of the interneurons to excite the presynaptic sympathetic efferents (Brown \& Weaver 2012, McGillivray et al 2009). In this manner, hypersensitive interneurons become the sole excitatory and inhibitory inputs of sympathetic efferents (McGillivray et al 2009). 


\section{Evaluation of Cardiovascular Function in patients with SCI}

In SCl, response to stress can be measured by inducing drops in BP to which the reflexes must respond (Previnaire et al 2010). Passive orthostasis, using either a head-up tilt test or a sit-up test, causes blood to pool in the viscera and lower limbs and quantifies the ability of the SNS to maintain BP without skeletal muscle contraction, allowing one to compare autonomic activity during stress to baseline autonomic tone (Sahota et al 2012). Analysis of HR variability (HRV) can illustrate autonomic control of the SA node during stress, while baroreflex sequence analysis can illustrate baroreceptor reflex sensitivity (BRS).

An analysis of HRV uses noninvasive measurements of continuous RRIs, gathered from an ECG, to measure autonomic control, or lack thereof, over the SA node. A "frequency domain" analysis, specifically a fast Fourier transform, deconstructs the oscillations and quantifies them by intensity, or "power," in order to determine the activity of the SNS and PNS (Baselli et al 1986, Ori et al 1992). Oscillations that occur within the low-frequency (LF; .04-.15 Hz) and highfrequency $(\mathrm{HF} ; .15-.40 \mathrm{~Hz}$ ) bands are important: upright, LF oscillations occur at a frequency of around 10-seconds $(.1 \mathrm{~Hz})$ and are the result of sympathetic activity; while supine they are a combination of sympathetic and parasympathetic activity. HF oscillations, supine and upright, are due to respiration and are vagally mediated (deBoer et al 1987, Inoue et al 1990, Ori et al 1992).

The baroreceptor sequencing technique attempts to evaluate baroreceptor activity by identifying consecutive "ramps" of increases or decreases in SBP that 
are three beats or more in length. A change in SBP that elicits a change in RRI is called a "sequence;" sequences are analyzed when the Pearson correlation coefficient between them is $>.80$. BRS, also called "BRS gain," of significant sequences is measured by quantifying the change of RRI in response to changes in SBP to get the slope in $\mathrm{ms} / \mathrm{mmHg}$ (Sagawa 2011). Despite the name, BRS slope does not reflect baroreceptor activity alone, but rather the integration of various reflex arcs that alter HR and vasoconstriction to maintain BP (including cardiopulmonary reflexes, baroreceptor reflexes, and the low-pressure receptors) (Scher et al 1991). Isolation of baroreceptor reflex activity requires either electrical or physical perturbation of the baroreceptors, a Valsalva maneuver, or pharmacological intervention (Hart et al 2010, Kamiya et al 2011, Vogel et al 2005). Up-up sequences, so-called because they involve an increase in SBP followed by an increase in $\mathrm{RRI}$, reflect the parasympathetic arc of baroreceptor reflex, while the down-down sequences reflect sympathetic activity and vagal withdrawal. A baroreceptor effectiveness index (BEI) can be calculated by dividing the number of significant sequences by the total number of sequences (Parati et al 2000, Zollei et al 2003). Supine, BRS slope largely reflects the vagal relationship between SBP and $\mathrm{RRI}$, as there is minimal sympathetic efferent vascular output beyond vasomotor tone (Scher et al 1991). While passively upright, however, the BRS measurements illustrate the increased sympathetic efferent activity to the heart and vasculature, as well as parasympathetic withdrawal (Blomqvist 2011). 
Induced orthostatic stress using a passive upright position demonstrates persons with tetraplegia not only have an impaired sympathetic response to stress, but that there is also diminished vagal withdrawal: LF oscillations decrease or are abolished while upright with no change in RRI HF oscillations (Houtman et al 2000, Inoue et al 1990, Wecht et al 2006). In SCI, BRS and BEI both decrease more while upright than non-injured controls (Claydon \& Krassioukov 2008, Houtman et al 2000, Wecht et al 2006). (It should be noted that not all of the decreases in BRS were significant, and another study of tetraplegics found that BRS actually increased while upright) (Munakata et al 2001). Further, studies have found that persons injured below T4 still exhibit decreased BRS and impaired LF oscillations while upright despite the presence of cardiac nerves (Castiglioni et al 2007, Legramante et al 2001, Wecht et al 2003).

Despite the ease with which this test is administered, decreased sympathetic output and reduced vagal withdrawal, coupled with the lack of skeletal muscle contraction, can create sustained decreases in BP while passively upright. Because of this, some favor other maneuvers to assess the integrity of the sympathetic and parasympathetic arcs. The Valsalva maneuver, a sustained airway pressure generation against a closed glottis, can illustrate the degree autonomic dysfunction in persons with $\mathrm{SCl}$ without redistribution of blood volume (Grimm et al 1998). Additionally, the influence of cardiopulmonary and low-pressure receptors are minimized, so the outcomes reflect the action of the 
baroreceptors and ANS alone (Gorlin et al 1957, Vogel et al 2005). The Valsalva maneuver is a sustained increase in intrathoracic pressure that impedes venous return. During the maneuver (called phase 2), there is a reflex tachycardia and vasoconstriction that maintain $\mathrm{BP}$ despite the increased intrathoracic pressure. Following the maneuver (phase 4), there is a SBP overshoot that inhibits vasoconstriction and causes a vagally-mediated reflex bradycardia (Palmero et al 1981, Persson \& Kirchheim 1991, Smith et al 1987). The magnitude of SBP overshoot is directly related to SNS activity during phase 2 (Vogel et al 2005). Respiratory rate and airway pressure are kept constant during the maneuver, while the increased intrathoracic pressure minimizes the distortion of the pulmonary vessels and the atria, preventing activation of the airway and lowpressure receptors, respectively (Porth et al 1984).

Cervical lesions are not well studied, but when compared to able-bodied persons, there is a significant decrease in reflex sympathetic tachycardia and vasoconstriction during phase 2 that eliminates the expected SBP overshoot during phase 4 (Frankel \& Mathias 1992, Mathias et al 1979). Upper thoracic lesions were found to have poor reflex responses during phases 2 and 4 , as well as decreased peak SBP values during phases 2 and 4, when compared to ablebodied persons (Houtman et al 1999). Absolute SBP values from low thoracic injuries were similar to able-bodied persons, but there were decreases in baroreceptor outcomes during phases 2 and 4, possibly due to inactivity or desensitization (Grimm et al 1998, Houtman et al 1999). 
Cardiovascular tests reveal, therefore, that persons with SCI not only experience cardiovascular dysfunction due to isolation of the spinal sympathetic neurons from the brainstem, but also due to downregulation of PNS activity. Imbalance of the ANS is further exacerbated by remodeling within the spinal cord. Management of cardiovascular dysfunction is therefore a primary goal in $\mathrm{SCl}$.

\section{MANAGEMENT OF SCI-INDUCED CARDIOVASCULAR AND PULMONARY COMPLICATIONS}

Much like evaluation of function, cardiovascular and pulmonary dysfunction are treated independently despite the close relationship between them and despite the reduced morbidity and mortality rates from concurrent treatment. Moreover, therapies that have the potential to improve cardiopulmonary outcomes, i.e., breathing therapies, are only assessed of pulmonary function outcomes.

Pulmonary therapies used to treat persons with $\mathrm{SCl}$ exist to augment the lack of respiratory motor activation; as such, higher injury levels require more intense interventions. High cervical lesions where the diaphragm is partially or fully paralyzed can require a mechanical ventilator, tracheostomy, or intubation in order to inspire; however, in addition to being invasive, these can lead to atrophy of the diaphragm due to disuse and create long-term dependencies. Increasingly, data demonstrate reduced risk of morbidity and mortality as persons with $\mathrm{SCl}$ are 
weaned off mechanical ventilation and replace it with positive pressure ventilation or inspiratory muscle pacing. While these therapies assist with normal respiration, there are still cough deficits due to paralysis of forced expiratory muscles. Many persons with SCI participate in cough therapies that include binders or manually assisted cough in order to mobilize secretions out of the airways and reduce the risk of pulmonary infections (Bach 2012, Galeiras Vazquez et al 2013, Zimmer et al 2007).

In addition to assisting with eupnea, there are therapies that aim to improve overall pulmonary function by strengthening the muscles. These therapies include targeted strengthening of inspiratory and expiratory muscles using a resistance-generating mouthpiece, electrical stimulation of the respiratory muscles, repeated maximal inspiratory and expiratory efforts, and singing lessons (Tamplin \& Berlowitz 2014, Van Houtte et al 2006). All have demonstrated some benefits to spirometric outcomes, airway pressure generation, and speech and singing ability. To date, there are no research studies that evaluate the effect of respiratory training on cardiovascular function in the $\mathrm{SCl}$ population. If respiratory training were effective in the $\mathrm{SCl}$ population it could be quite beneficial, because management of cardiovascular dysfunction in $\mathrm{SCl}$ is limited.

Largely, management of cardiovascular dysfunction in SCI involves management of BP dysfunction; to date, there are few therapy options that consistently improve cardiovascular function. To manage low-resting BP, persons 
with $\mathrm{SCl}$ frequently employ abdominal binders and leg compression stockings to increase venous return and decrease blood pooling in the periphery (Phillips \& Krassioukov 2015). Incidentally, abdominal binders have the added benefit of improving pulmonary function (Wadsworth et al 2009). Therapies like armcycling, and electrical stimulation of leg-muscles can increase BP values but are inconsistent at preventing $\mathrm{OH}$ (Gillis et al 2008, Mills et al 2015). There are pharmacological therapies to help manage BP, but drugs that decrease frequency of $\mathrm{OH}$ by increasing resting $\mathrm{BP}$ can exacerbate bouts of $\mathrm{AD}$, while those that decrease risk of $A D$ can exacerbate low-resting BP and $\mathrm{OH}$ (Phillips \& Krassioukov 2015).

There is therefore a gap in targeted cardiopulmonary therapies that needs to be addressed in this population: management of dysfunction is possible for both pulmonary and cardiovascular deficits, but currently there are no therapies able to improve cardiovascular function. The most promising candidate to fill this gap is respiratory motor training (RMT): it is proven effective at improving pulmonary function outcomes, which in $\mathrm{SCl}$ have been shown to be related to improved cardiovascular parameters. Application of the therapy itself would engage all of the cardiovascular reflexes mediated by respiration, which would activate available efferent cardiac and vasomotor nerves. Moreover, respiratory training has demonstrated significant cardiopulmonary improvements in NI population when used to treat persons suffering from heart failure or stroke (Chiappa et al 2008, Segal et al 2013, Smart et al 2012, Sutbeyaz et al 2010). 
This training has great potential toward not only improving cardiopulmonary outcomes, but treating the leading causes of morbidity and mortality in this population. 


\section{CHAPTER II:}

\section{HYPOTHESES AND SPECIFIC AIMS}

The relationship between cardiovascular and pulmonary function in the non-injured population led us to believe that, not only is this relationship still present in spinal-cord injury (SCl), but that this relationship could exist independent of level and completeness of injury. Pulmonary function correlates strongly with level of injury, but cardiovascular dysfunction does not. Therefore we think an additional confounder to the relationship between cardiovascular function and $\mathrm{SCl}$ is pulmonary function: in addition to the dysfunction caused by level and completeness of injury, there is dysfunction that exists due to the poor respiratory motor function. The alternative to this is any cardiovascular dysfunction independent of level and completeness of injury has the potential to be reversed. Thus, our broad hypothesis is improvement to pulmonary function has the potential to improve cardiovascular function in persons with $\mathrm{SCl}$ irrespective of level and completeness of injury.

Our first aim was to investigate the relationship between pulmonary function, cardiovascular function, and $\mathrm{SCl}$ using a variety of outcomes in order to elucidate the mechanisms that cause cardiovascular dysfunction. We 
hypothesized that there would be significant deficits to pulmonary and cardiovascular function in persons with $\mathrm{SCl}$ compared to non-injured persons. The impaired respiratory motor control will decrease the activity of the respiratory pump, and the cardiovascular reflexes that depend on respiration, subsequently decreasing cardiovascular regulation.

Our second aim was to examine the relationship between level of injury and pulmonary function, and level of injury and cardiovascular function. If pulmonary and cardiovascular function are impaired, but pulmonary function depends on injury while cardiovascular function does not, the relationships between outcomes and level and completeness of injury will demonstrate this. Our second hypothesis is that respiratory motor function will correlate with level and completeness of injury, while cardiovascular function outcomes will not; level and completeness of injury will thus be poor predictors for cardiovascular regulation due to the additional dysfunction caused by poor respiratory motor control.

Our third and final aim was to examine the changes that occur to cardiovascular regulation after improvements to pulmonary function. If hypotheses one and two are correct, and cardiovascular regulation is indeed impaired by poor respiratory motor control, then any improvement to respiratory motor control should improve cardiovascular function outcomes. We therefore hypothesize that the dependence of cardiovascular function on respiratory motor 
function will cause cardiovascular outcomes to improve once pulmonary function outcomes improve. 


\section{CHAPTER III:}

\section{METHODS AND MATERIALS}

\section{Criteria}

Inclusion criteria for participation in the control and intervention spinal cord injury (SCl) groups were as follows: 18 years of age or older; chronic injuries six months or more from date of injury; cervical and thoracic neurological injuries, incomplete and complete, as identified by an American Spinal Injury Assessment (ASIA) performed by a clinician (AIS A-D); and ventilator independent, with a pulmonary deficit of at least $15 \%$. Exclusion criteria were the presence of endocrine, cardiovascular, gastrointestinal, or pulmonary diseases not-related to the $\mathrm{SCl}$, and pregnancy.

Inclusion criteria for non-injured participants were non-smokers, between 18 and 55 years of age; stable medical condition; and the absence of cardiopulmonary diseases.

\section{Recruitment}

$\mathrm{SCl}$ participants were recruited directly from the outpatient pool at Frazier Rehabilitation Institute, or were recommended to participate in the study by a 
study clinician. Non-injured participants were recruited by flyers posted on the University of Louisville campus and ads placed in the daily University-wide newsletters. After participants were consented and fully informed about the risks and benefits of this research project, SCI participants were then screened to ensure they met the required inclusion criteria by a clinician (an ASIA exam and a clinical exam) and by screening assessments (discussed later).

\section{Participants}

There were 58 persons that participated in this study. Eleven were noninjured, with a mean age of $36 \pm 14$ (Table III-1). Seven of the non-injured participants were female, and 4 were male. There were 47 persons with spinalcord injury that participated in the study, 25 in the respiratory motor training (RMT) group and 22 in the control group (no RMT). Mean ages of the RMT and control groups were similar at $41 \pm 12$ years and $44 \pm 14$ years, respectively. The RMT group had 16 participants with a cervical injury, 8 motor-complete (AIS A or B) injuries and 8 motor-incomplete (AIS C or D) injuries. Of the 9 thoracic injuries in the RMT group, 4 were motor complete (AIS A, only) and 5 were motorincomplete (AIS C, only) (Table III-2). The control group had 13 cervical injuries and 9 thoracic injuries. Seven of the cervical injuries were motor-complete (AIS A or B) while 6 were motor-incomplete (AIS C or D). One thoracic control participant was motor-incomplete (AIS D) while the rest were motor-incomplete (AIS A) (Table III-3). 
All persons that were recruited to participate in the study were assessed of baseline values during screening experiments (called the pre-test experiment). Participants that were in the RMT group participated in the RMT protocol for 4 weeks, and the day after termination of RMT were assessed of their post-test values. Participants in the control group were assessed of post-test values 4-6 weeks after the pre-test, and did not undergo RMT or other clinical therapies (locomotor training, functional electrical stimulation, etc.) in the interim that would potentially affect the evaluation of cardiovascular outcomes. 
Table III-1: Demographics of Non-injured group

\begin{tabular}{ccccc} 
& Age & Sex & $\begin{array}{c}\text { Height } \\
\text { (in) }\end{array}$ & $\begin{array}{c}\text { Weight } \\
\text { (lbs) }\end{array}$ \\
\hline \hline N116 & 31 & F & 62 & 110 \\
N117 & 55 & F & 68 & 132 \\
N118 & 32 & F & 63 & 120 \\
N119 & 64 & M & 73 & 190 \\
N120 & 27 & M & 70 & 170 \\
N121 & 32 & M & 73 & 175 \\
N122 & 25 & M & 77 & 180 \\
N123 & 27 & F & 63 & 125 \\
N124 & 29 & M & 74 & 206 \\
N84 & 29 & F & 63 & 245 \\
N125 & 27 & M & 69 & 172 \\
\hline Mean \pm SD & $34 \pm 12$ & NA & $68 \pm 41$ & $166 \pm 41$
\end{tabular}

Non-injured participants were recruited from the University of Louisville, and were mostly faculty, staff, and students. The mean age was $34 \pm 12$ years of age, with a height of $68 \pm 41$ inches, and a weight of $166 \pm 41$ pounds. There were no significant differences between demographics of $\mathrm{NI}$ and spinal cordinjured participants. Values are represented as mean \pm standard deviation. 
Table III-2: Demographics of Respiratory Motor Training Group

\begin{tabular}{|c|c|c|c|c|c|c|c|}
\hline ID & Age & Sex & $\begin{array}{l}\text { Height } \\
\text { (in) }\end{array}$ & $\begin{array}{c}\text { Weight } \\
\text { (lbs) }\end{array}$ & LOI & ASIA & $\begin{array}{c}\text { Months } \\
\text { since } \\
\text { Injury }\end{array}$ \\
\hline A58 & 40 & $M$ & 70 & 230 & C3 & $A$ & 22 \\
\hline A38 & 37 & $F$ & 69 & 130 & C4 & A & 248 \\
\hline A65 & 29 & $M$ & 68 & 180 & C4 & A & 10 \\
\hline B18 & 56 & $M$ & 72 & 155 & C3 & B & 29 \\
\hline B06 & 42 & $\mathrm{~F}$ & 67 & 123 & C4 & B & 70 \\
\hline B11 & 25 & $M$ & 70 & 185 & C4 & B & 98 \\
\hline B16 & 60 & $M$ & 71 & 220 & C4 & B & 31 \\
\hline B19 & 40 & $M$ & 74 & 177 & C6 & B & 6 \\
\hline C33 & 59 & $M$ & 69 & 145 & $\mathrm{C} 2$ & C & 532 \\
\hline C27 & 58 & $M$ & 70 & 190 & C4 & C & 47 \\
\hline С30 & 19 & $\mathrm{~F}$ & 67 & 94 & C4 & C & 12 \\
\hline C34 & 20 & $M$ & 76 & 140 & C4 & C & 53 \\
\hline C26 & 33 & $M$ & 72 & 165 & C6 & C & 4 \\
\hline C38 & 42 & $M$ & 70 & 245 & $\mathrm{C} 2$ & D & 156 \\
\hline B17 & 59 & $M$ & 75 & 217 & C4 & D & 15 \\
\hline C18 & 31 & $M$ & 71 & 214 & C4 & D & 36 \\
\hline A55 & 35 & $M$ & 68 & 165 & $\mathrm{~T} 11$ & A & 50 \\
\hline A46 & 47 & $\mathrm{~F}$ & 62 & 192 & T6 & A & 43 \\
\hline A66 & 48 & $M$ & 72 & 170 & T6 & A & 65 \\
\hline A75 & 49 & $M$ & 73 & 188 & T9 & A & 84 \\
\hline C16 & 35 & $M$ & 72 & 185 & T1 & C & 70 \\
\hline C24 & 40 & $F$ & 68 & 125 & $\mathrm{~T} 11$ & C & 110 \\
\hline C25 & 37 & $M$ & 70 & 195 & $\mathrm{~T} 11$ & C & 32 \\
\hline C39 & 45 & $\mathrm{~F}$ & 68 & 135 & $\mathrm{~T} 11$ & C & 21 \\
\hline C28 & 29 & $\mathrm{M}$ & 70 & 160 & T5 & C & 56 \\
\hline ean $\pm S D$ & $40 \pm 12$ & NA & $70 \pm 3$ & $173 \pm 37$ & NA & NA & $76 \pm 109$ \\
\hline
\end{tabular}

There were 25 participants in the respiratory motor training group, 16 of which had cervical injuries. Twelve participants had motor complete injuries (AIS A or B), while 13 had motor-incomplete injuries (AID C or D). The majority (19) of the participants were male. Mean age was $41 \pm 12$ years. Height was $70 \pm 3$ inches, while weight was $173 \pm 37$ pounds. Mean duration of injury at the time of pre-test assessment (in months) was $76 \pm 109$. Values are represented as mean \pm standard deviation 
Table III-3: Demographics of Control Group

\begin{tabular}{cccccccc} 
ID & Age & Sex & $\begin{array}{c}\text { Height } \\
\text { (in) }\end{array}$ & $\begin{array}{c}\text { Weight } \\
\text { (lbs) }\end{array}$ & LOI & ASIA & $\begin{array}{c}\text { Months } \\
\text { since } \\
\text { Injury }\end{array}$ \\
\hline \hline A33 & 58 & M & 74 & 232 & C3 & A & 20 \\
A38 & 39 & F & 69 & 113 & C4 & A & 237 \\
A64 & 49 & M & 75 & 155 & C4 & A & 405 \\
B21 & 30 & M & 73 & 165 & C4 & B & 64 \\
B22 & 51 & M & 70 & 180 & C4 & B & 37 \\
B11 & 25 & M & 70 & 185 & C5 & B & 98 \\
B20 & 28 & M & 65 & 128 & T2 & B & 53 \\
C14 & 47 & M & 76 & 130 & C3 & C & 10 \\
C41 & 37 & M & 70 & 140 & C4 & C & 171 \\
C48 & 39 & M & 68 & 163 & C6 & C & 113 \\
D35 & 60 & M & 71 & 220 & C2 & D & 35 \\
C18 & 35 & M & 71 & 214 & C4 & D & 57 \\
D42 & 72 & M & 71 & 230 & C5 & D & 33 \\
A35 & 55 & M & 73 & 200 & T3 & A & 377 \\
A36 & 56 & M & 68 & 210 & T3 & A & 341 \\
A57 & 27 & F & 73 & 165 & T4 & A & 45 \\
A69 & 26 & M & 73 & 140 & T4 & A & 27 \\
A39 & 36 & M & 70 & 175 & T5 & A & 185 \\
A53 & 30 & M & 70 & 141 & T5 & A & 23 \\
A73 & 28 & M & 67 & 180 & T6 & A & 58 \\
A74 & 56 & M & 69 & 240 & T9 & A & 418 \\
D41 & 44 & F & 65 & 180 & T8 & D & 50 \\
\hline Mean \pm SD & $41 \pm 12$ & NA & $71 \pm 3$ & $171 \pm 36$ & NA & NA & $129 \pm 136$
\end{tabular}

There were 22 participants in the control group, 13 with a cervical injury and 9 with a thoracic injury. The majority were male (19) with motor-complete (AIS A or B; 15 total) injuries. The mean age was $44 \pm 14$ years at the time of pre-test assessment, with a mean injury duration of $129 \pm 136$ months. Height was $71 \pm 3$ inches, while weight was $171 \pm 36$ pounds. Values are represented as mean \pm standard deviation. 


\section{Equipment}

All recorded data was converted from analog to digital signals using a Powerlab 16/35 system, connected to a computer running LabChart 7 (AD Instruments, Denver, CO). Forced vital capacity (FVC) and Forced expiratory volume in $1 \mathrm{~s}\left(\mathrm{FEV}_{1}\right)$ were recorded using a CPFS/D USB spirometer and BreezeSuite System (MGC Diagnostics, St. Paul, MN). Percent-predicted values from BreezeSuite were calculated by comparing the SCI participant's values in L/s to an age-, height-, weight- and sex-matched outcome from a non-injured person. Recording MEP (in $\mathrm{cmH}_{2} \mathrm{O}$ ) required an MP45, low-pressure transducer system (Validyne Engineering, Northridge, CA) connected to a t-piece monitoring circuit with a flexible air-tube. The t-piece consisted of a mouthpiece into which the participant breathed, a valve used to resist expiration and generate pressure, and a small leak $1.5 \mathrm{~mm}$ in diameter to prevent recruitment of buccal muscles and glottis closure during the event. For the Valsalva maneuver, there was an additional tube that connected to an analog pressure gauge so the participant could monitor their airway pressure.

Blood pressure, HR, and respiration kinematics were recorded continuously using a Finometer MIDI, ECG bioamplifier (Finapres Medical Systems, The Netherlands), and Pneumotrace respiratory belt transducer (AD Instruments, Denver, CO). A finger cuff with an air bladder and infrared plethysmograph measured continuous finger arterial pressure from either the middle finger or thumb of the left hand, while a three-lead ECG connected to a 
bioamplifier monitored HR and RRI. The arterial finger waveform was reconstructed to a brachial waveform by the Finometer MIDI so that the finger cuff SBP and DBP values could be calibrated to a brachial BP reading from the right arm (GE's Dinamap Patient Monitor, Boston, MA). Finally, a pneumotrace respiratory belt was placed around the chest and abdomen to determine inspiration and expiration.

\section{RESPIRATORY MOTOR CONTROL ASSESSMENT}

Spirometry, maximum airway pressure generation and the Valsalva maneuver were recorded in the seated position during a respiratory motor control assessment (RMCA). Participants performed the pulmonary function tests while $\mathrm{BP}$ and HR were continuously monitored. The participant was informed by the instructor how to perform each maneuver verbally and then watched as the instructor demonstrated with the respective mouthpieces. Each event was performed three times, with poor attempts (i.e., those with spasms, coughs, or yawns) excluded and repeated. Prior to the first attempt of each maneuver, finger cuff BP values were calibrated to a brachial BP reading by GE Dinamap.

\section{Spirometry}

To measure FVC and $\mathrm{FEV}_{1}$, the participant wore a nose-clip and was instructed to expire maximally and rapidly from total lung capacity (TLC). An 
advocate placed the nose-clip and held the mouthpiece to minimize extraneous effort during the maneuver. FVC measures the amount of air that can be forcefully expired in $\mathrm{L} / \mathrm{s}$, while $\mathrm{FEV}_{1}$ measures the amount of air expired during the first second of the FVC maneuver. In SCI participants, both effectively assess pulmonary function as well as respiratory motor control (Ovechkin et al 2010, Schilero et al 2009). The spirometric outcomes were recorded as percentpredicted values instead of flow, which controlled for discrepancies between persons due to age, sex, height, or weight. Each event was attempted three times, with at least 30 s rest in between events to allow BP values to return to baseline. Attempts were averaged for each maneuver. Participants were instructed to breathe normally and refrain from inessential talking or movement between attempts.

\section{Maximum Airway Pressure Generation}

A MEP measures the maximum airway pressure (in $\mathrm{cmH}_{2} \mathrm{O}$ ) that a participant can generate. In SCl, it can indicate deficits in pulmonary function and respiratory motor control (Ovechkin et al 2010). Participants wore a nose-clip and were instructed to inspire to TLC through the mouthpiece, and then expire maximally into the mouthpiece. An advocate placed the nose-clip and held the mouthpiece to eliminate any extraneous effort by the participant. Each event was repeated three times, with 30 s minimum in between events to allow BP to return to baseline values. Participants were instructed to breathe normally and refrain 
from inessential movement or talking between attempts. The MEP value is obtained by averaging $\pm .5 \mathrm{~s}$ around the absolute peak during each attempt. Attempts that varied by $<20 \%$ were averaged for each participant. Additionally, attempts that triggered coughs, spasms, etc., were excluded from analysis.

\section{Valsalva Maneuver}

The Valsalva maneuver is a sustained increase in intrathoracic pressure that creates predictable and repeatable changes in BP and HR used to assess cardiovascular function. Cardiovascular response to the Valsalva maneuver is categorized into four phases. Early and late phase II assess HR response and vascular response, respectively, to a rapid drop in BP. Phase IV assesses the HR response to a rapid increase in BP. BRS assessment of these phases (in $\mathrm{ms} / \mathrm{mmHg}$ ) has been demonstrated reliable in assessing cardiovascular regulation in SCl (Gorlin et al 1957, Grimm et al 1998, Porth et al 1984). Participants wore a nose-clip and were instructed to inspire to TLC through a mouthpiece held by an advocate; at TLC, they were instructed to sustain airway pressure at $30 \mathrm{cmH}_{2} \mathrm{O}$ for 20 s by watching an analog manometer held at eyelevel by an advocate. The instructor notified participants of each second that passed. Participants were encouraged to sustain for 20s, but were told to end the maneuver early if they felt any symptoms of presyncope (i.e., dizziness, blurry or spotty vision, or nausea). Additionally, the maneuver was terminated by the instructor if BP dropped below 70/40mmHg or if HR exceeded 150BPM. 
Maneuvers were repeated three times, provided no attempt triggered syncope and the participant felt confident to continue. There was one to three minutes of silent rest between attempts.

BRS slope of early phase II, late phase II and phase 4 was calculated using SBP values and RRIs. Phases were identified visually within LabChart according to standards set by Gorlin and colleagues: early phase II was peak SBP until lowest SBP value during the maneuver; late phase II was the lowest SBP value until termination of the maneuver; phase IV was the lowest SBP until the peak SBP following the maneuver (Gorlin et al 1957). Provided no attempts triggered spasms, coughs, etc., SBP and RRI values were extracted and analyzed with SBP plotted against the RRI that followed; to ensure baroreceptor engagement, the beginning of each sequence analyzed included three consecutive increases or decreases in RRI (Palmero et al 1981). A linear regression and Pearson correlation coefficient of SBP and RRI sequences were calculated in R (R Foundation for Statistical Computing, Vienna, Austria). BRS slopes $(\mathrm{ms} / \mathrm{mmHg})$ of the linear regressions were included for comparison provided the Pearson correlation coefficient was $>.80$. Mean values of acceptable slopes were included in the analysis.

\section{Sustained Maximum Airway Pressure Generation}

To perform the 5s MEP, an advocate placed a nose-clip and held the mouthpiece to minimize extraneous effort by the participant. The participant was 
then instructed to inspire to TLC through the mouthpiece, and then expire maximally through the mouthpiece for the duration of an audible tone. Each attempt was repeated three times, with 30 s of quiet rest in between each attempt. The participant was instructed to refrain from inessential talking or movement during the recovery.

Additionally, we attempted to validate the cardiovascular response following the 5s MEP. BRS slopes were calculated by visually inspecting the sequences during and following the 5 s MEP using the same criteria as the Valsalva maneuver: the sequence during the 5 s MEP started with the peak SBP and ended with the lowest SBP during the maneuver, while the sequence following the 5s MEP started with the lowest SBP following the maneuver and ending with the peak SBP. Normal events (i.e., events without spasms, coughs, etc.) were extracted of SBP and RRI values and imported into $R$ for analysis. Sequences started with three or more consecutive increases or decreases in RRI; RRI was matched to the SBP value that preceded it. A linear regression and Pearson correlation coefficient of the sequences was calculated. BRS slopes $(\mathrm{ms} / \mathrm{mmHg})$ from the linear regression were included for comparison when the Pearson correlation coefficient was $>.80$. Mean BRS Slope of each acceptable attempt was included in the analysis. 


\section{ORTHOSTATIC STRESS TEST}

Orthostatic stress test was performed in the morning, between 9:00 and 10:30 AM, in a dimly lit, temperature controlled room $\left(22^{\circ} \mathrm{C}\right)$. Participants were asked to eat a light breakfast (i.e., little to no sugar or fat), refrain from caffeine 12 hours prior to the experiment, and empty their bladder prior to arrival. Prior to instrumentation, participants were introduced to the equipment and reminded of the experiment protocol. Experiments were rescheduled if the participant complained of excess or unusual pain on that particular morning. Participants were recumbent upon a Hausted Manual Gurney Chair (GF Health Products, Atlanta, GA) with a belt around their chest to prevent muscle recruitment during the position change. Whilst supine, participants were instrumented with a finger cuff, height correction unit, and front-end unit of a Finometer MIDI (Finapres Medical Systems, The Netherlands); a three-lead ECG bioamplifier using RA, RL, and LL leads (Finapres Medical Systems, The Netherlands); and two Pneumotrace respiratory belts (AD Instruments, Denver, $C O$ ). Finger cuff BP values were calibrated to a brachial arm reading using a GE Dinamap Patient Care Monitor (GE Healthcare, Boston, MA). Respiratory belts were calibrated to each participant before the start of the test by instructing the participant to take three deep breaths (i.e., vital capacity) and three quick breaths (each $<1$ s).

Once equipment was calibrated, the participant was given five minutes of quiet rest to acclimate to the equipment. Once the study began, the participant 
was instructed to refrain from inessential movement or speech for the duration of the study. The participant was supine for 15 minutes, passively moved to a seated position for 15 minutes, and then moved to supine for 10 minutes. There were regular brachial arm readings at time points 0:03, 0:13, 0:18, 0:28, and $0: 33$, and additional readings whenever the experiment leader felt they were necessary. The test was terminated if the participant experienced autonomic dysreflexia, uncontrolled orthostatic hypotension that would lead to syncope, or if the participant felt too uncomfortable to continue.

Following the experiment, $\mathrm{BP}$ values that did not match arm readings were calibrated within LabChart 7 using the "Unit Conversion" function. Absolute BP and $\mathrm{HR}$ values were obtained using LabChart 7. For all other analyses, BP and ECG waveforms were exported into Matlab (Mathworks, Natick, MA).

\section{Mean Values}

Systolic BP, DBP, RRI and HR values were obtained in LabChart following comparison of Finometer values to GE Dinamap values. Values were averaged for minutes 0:03 to 0:13 of supine, and 0:18 and 0:28 of seated. Deep breaths, coughs, spasms, or yawns were excluded from the average. SBP and DBP values were obtained from the LabChart BP Analysis Module, which analyzes the BP waveform obtained from the Finometer MIDI. RRI and HR values were obtained from the ECG waveform using the LabChart ECG Analysis Module. 
Heart Rate Variability

Heart rate variability is an analysis of oscillations in RRI to determine balance of SNS and PNS at rest (i.e., whilst supine) and during stress (i.e., whilst seated)(Inoue et al 1995). In Matlab, a program was created to apply a power spectral analysis (specifically, a Fast-Fourier Transform) of RRI oscillation using a fixed-time interval. Supine and seated positions were analyzed from minutes $0: 03$ to $0: 13$, and $0: 18$ to $0: 28$, respectively. RRI power in $\mathrm{ms}^{2}$ was reported for low- and high-frequency bands.

\section{Baroreceptor Sequencing}

Baroreceptor Sequence analysis is performed in Matlab. It evaluates spontaneous baroreflex activity while supine and seated to assess cardiovascular regulation in response to stress(Parati et al 2000). Consecutive ( $\geq$ 3 beats) increases or decreases in SBP are identified and plotted against the RRI that follows using a linear regression; SBP and $\mathrm{RRI}$ ramp sequences that have a

$\mathrm{R}^{2}>.85$ are considered significant and used to evaluate baroreceptor activity. The slope (in $\mathrm{ms} / \mathrm{mmHg}$ ) of significant sequences is reported, as well as the number of significant ramp sequences that occur during the period analyzed, which describes the activity and sensitivity of the baroreceptors. Ramp sequences where a SBP increase is followed by an increase in RRI, called Up- 
Up Ramps, are indicative of vagal activity. Decreased SBP ramps followed by decreased RRI (Down-Down Ramps) are a result of the SNS. In addition to sensitivity, the effectiveness of the baroreceptors (Baroreceptor effectiveness index, or $\mathrm{BEI}$ ) can be determined by dividing the number of significant ramp sequences in to the total number of SBP ramps during the period analyzed.

\section{STATISTICAL METHODS}

All data were analyzed in R (R Foundation for Statistical Computing, Vienna, Austria). Data in bar plots are represented as mean $\pm \mathrm{SD}$; box and whisker plots contain $95 \%$ of observations, with the box encompassing $25-75 \%$ of data. Outliers were defined and excluded at 3SD (Iglewicz \& Hoaglin 1993) from the mean. Significance was set to $a=.05$. The presence of multiple covariates in the baseline and pre-post data and normally distributed residuals made the linear regression an appropriate test for significance (Vittinghoff 2012).

To compare functional outcomes of the SCI group to NI participants for hypotheses one and two, univariate and multiple linear regression models were created in order to illustrate the complex and multifaceted ways an injury can affect cardiovascular and pulmonary function. Thus participants were grouped not only into injured and non-injured, but also by groups that included the level of injury and AIS impairment (Table III-4) in order to illustrate the effects, both combined and in isolation, on pulmonary and cardiovascular function. 
To compare pre- to post-test outcomes for hypothesis three, change scores were calculated for each participant by subtracting their post-test score from their pre-test score. This new measure was used as the dependent variable in all regression models. Change score of the control group was the reference to which RMT was compared. To determine the influence of injury on change preto post-test, injuries were grouped to include level of injury and AIS impairment (Table III-5). 
Table III-4: Univariate and Multiple Linear Regression Models Comparing Injured and Non-Injured Outcomes

\begin{tabular}{c|l} 
Model Number & \multicolumn{1}{l}{ Formula } \\
\hline \hline 1-A & $y_{i}=\beta 0_{i}+\beta($ SCI $)+\varepsilon_{i}$ \\
$2-A$ & $y_{i}=\beta 0_{i}+\beta$ (Cervical) $+\beta$ (Thoracic) $+\varepsilon_{i}$ \\
$3-A$ & $y_{i}=\beta 0_{i}+\beta($ LOI $)+\varepsilon_{i}$ \\
$4-A$ & $y_{i}=\beta 0_{i}+\beta($ AIS B $)+\beta($ AIS C/D $)+\varepsilon_{i}$ \\
$5-A$ & $y_{i}=\beta 0_{i}+\beta($ SCI $)+\beta($ AIS B $)+\beta($ AIS C/D $)+\varepsilon_{i}$ \\
$6-A$ & $y_{i}=\beta 0_{i}+\beta($ Cervical $)+\beta($ Thoracic $)+\beta(A I S$ B $)+\beta($ AIS C/D $)+\varepsilon_{i}$ \\
$7-A$ & $y_{i}=\beta 0_{i}+\beta($ LOI $)+\beta($ AIS B $)+\beta($ AIS C/D $)+\varepsilon_{i}$
\end{tabular}

For all models with injury as a factored variable, non-injured persons were the reference group. Model 1-A grouped all injured persons together ( $\mathrm{SCl})$, while 2-A grouped injured participants by cervical and thoracic injuries. Model 3-A ordered level of injury from $\mathrm{C} 2$ to $\mathrm{T} 12(\mathrm{C} 2=1, \mathrm{C} 3=2$, etc. $)$ as an interval variable (LOI). Model 4-A grouped participants by AIS impairment (AIS A, B, C, or $\mathrm{D}$, as determined by an ASIA exam). Models 5-A, 6-A, and 7-A grouped participants by $\mathrm{SCl}$, cervical and thoracic injuries, and level of injury, respectively, while controlling for AIS impairment. 
Table III-5: Univariate and Multiple Linear Regression Models Comparing RMT and Control Groups

\begin{tabular}{|c|c|}
\hline Model Number & Formula \\
\hline $1-\mathrm{B}$ & $y i=\beta 0 i+\beta(\Delta R M T)+\varepsilon i$ \\
\hline $2-B$ & $y i=\beta 0 i+\beta(\Delta R M T)+\beta($ Thoracic $)+\varepsilon i$ \\
\hline $3-B$ & $y i=\beta 0 i+\beta(\Delta R M T)+\beta($ Thoracic $)+\beta($ AIS B $)+\beta($ AIS C/D $)+\varepsilon i$ \\
\hline $4-B$ & $y i=\beta 0 i+\beta(\Delta R M T-C e r v i c a l)+\varepsilon i$ \\
\hline $5-B$ & $y i=\beta 0 i+\beta(\Delta R M T)+\beta($ LOI $)+\varepsilon i$ \\
\hline $6-\mathrm{B}$ & $y i=\beta 0 i+\beta(\Delta R M T)+\beta($ LOI $)+\beta($ AIS B $)+\beta($ AIS C/D $)+\varepsilon i$ \\
\hline
\end{tabular}

For all models with training as a factored variable, non-trained persons were the reference group. Model 1-B compared Respiratory Motor Training (RMT) group to control without controlling for injury. Model 2-B compared RMT to control groups while grouping participants by cervical or thoracic injuries. Model 3-B included a grouping factor for both cervical/thoracic injuries as well as for AIS score (AIS A, B, C, or D, as determined by an ASIA exam). Model 4-B included only persons with a cervical injury in the RMT and control groups. Level of injury (LOI) in model 5 -B was ordered from $\mathrm{C} 2$ to $\mathrm{T} 12(\mathrm{C} 2=1, \mathrm{C} 3=2$, etc. $)$ and considered an interval variable. Model 6-B included a factor for LOI while also including AIS impairment. 


\section{CHAPTER IV:}

\section{EVALUATION OF BARORECEPTOR SENSITIVITY}

\section{INTRODUCTION}

First, we found that $70 \%$ of participants could not perform the Valsalva maneuver: they were incapable of sustaining the required pressure for 20 s, or experienced presyncope and couldn't safely continue (Table IV-1). The Valsalva maneuver was our method for assessing the baroreceptor reflex in isolation: despite the name, baroreceptor sequencing includes the activity of cardiopulmonary and low-pressure receptors, and as such it is actually an assessment of overall cardiovascular regulation. We therefore decided to validate a new maneuver to replace the baroreceptor sensitivity (BRS) results obtained by the Valsalva maneuver. We used a maximum airway pressure generation, sustained for 5-7s, to substitute for the Valsalva maneuver. For brevity, it was called a 5s MEP.

If there were no significant differenced detected between methods it would mean 5s MEP maneuver was long enough to substitute for early phase II of the Valsalva maneuver (Vogel et al 2005), and the increased intrathoracic pressure was sufficient to simulate a Phase IV. Thus both maneuvers would activate the 
reflex tachycardia and bradycardia needed to assess BRS. The 5s MEP maneuver was not long enough, however, to recruit the vasculature, and thus there was nothing similar to a late phase II.

\section{METHODS}

The 5s MEP maneuver and the Valsalva maneuver were obtained during a Respiratory Motor Control Assessment (RMCA, 42). We analyzed the phases during and following the 5s MEP maneuver using the same methods required for the Valsalva maneuver in order to minimize bias (see Valsalva Maneuver, 44). Phases II and IV of the Valsalva maneuver were compared to BRS slopes (in $\mathrm{ms} / \mathrm{mmHg}$ ) obtained during and following the 5s MEP maneuver, respectively. A repeated measures ANOVA was used to test for significance in those participants that were able to successfully complete the Valsalva maneuver $(n=$ 14, Table IV-1). Values are reported as mean \pm standard deviation.

\section{RESULTS}

A repeated measures, two-way ANOVA found no significant difference $(F=.02 ; d f=1 ; p=.90)$ between the slopes of early phase II and phase IV of the Valsalva maneuver and the slope during and following the 5s MEP maneuver, respectively (Table IV-2). 
Table IV-1: Mean Outcomes of Valsalva Maneuver

\begin{tabular}{ccccc} 
Performance & Injury & Number & $\begin{array}{c}\text { Pressure } \\
\left(\mathrm{cmH}_{2} \mathrm{O}\right)\end{array}$ & $\begin{array}{c}\text { Duration } \\
(\mathrm{s})\end{array}$ \\
\hline \hline $\begin{array}{c}\text { Did not } \\
\text { complete }\end{array}$ & Cervical & 22 & $21 \pm 5$ & $17 \pm 3$ \\
& Thoracic & 8 & $25 \pm 5$ & $19 \pm 2$ \\
\multirow{2}{*}{ Completed } & Cervical & 5 & $34 \pm 7$ & $20 \pm 0$ \\
& Thoracic & 9 & $37 \pm 4$ & $20 \pm 0$
\end{tabular}

Of the 39 participants that attempted the Valsalva maneuver, only 14 sustained the required pressure for 20s; the remainder experienced episodes of presyncope that made it too difficult to continue, or lacked the strength to sustain the maneuver. Values are represented as mean \pm standard deviation. 
Table IV-2: ANOVA Results Comparing Valsalva Maneuver to Sustained, Maximum Expiratory Pressure Maneuver

\begin{tabular}{|c|c|c|}
\hline \multirow[b]{2}{*}{ 5s MEP } & \multicolumn{2}{|c|}{ Valsalva Maneuver } \\
\hline & $\begin{array}{c}\text { Phase II } \\
3.3 \pm 2 \\
\mathrm{~ms} / \mathrm{mmHg}\end{array}$ & $\begin{array}{c}\text { Phase IV } \\
5.1 \pm 2 \\
\mathrm{~ms} / \mathrm{mmHg}\end{array}$ \\
\hline $\begin{array}{c}\text { During } \\
2.7 \pm 2 \\
\mathrm{~ms} / \mathrm{mmHg}\end{array}$ & NS & $p<.05$ \\
\hline $\begin{array}{c}\text { Following } \\
4.9 \pm 2 \\
\mathrm{~ms} / \mathrm{mmHg}\end{array}$ & $p<.05$ & NS \\
\hline
\end{tabular}

A two-way, repeated measures ANOVA found no significant differences between method and BRS slope of phase II of the Valsalva maneuver and BRS slope during the 5s MEP, nor between BRS slope of phase IV of the Valsalva maneuver and BRS slope following the 5s MEP. Values are represented as mean \pm standard deviation. 


\section{DISCUSSION}

The slopes $(\mathrm{ms} / \mathrm{mmHg})$ generated by the $5 \mathrm{~s}$ MEP maneuver were not significantly different than the slopes obtained by the Valsalva maneuver, thus it can be inferred that the 5s MEP maneuver elicits the same tachycardia and bradycardia one would expect to find during the Valsalva maneuver. Because it was a valid replacement and easier for our participants to perform, we decided to use the 5s MEP to evaluate BRS in our SCI participants. 


\title{
CHAPTER V:
}

\section{MECHANISMS OF RESPIRATORY AND}

\section{CARDIOVASCULAR DYSFUNCTION IN SPINAL CORD}

\author{
INJURY
}

\section{INTRODUCTION}

Our first aim was to investigate respiratory motor function and cardiovascular regulation and their respective roles in spinal cord injury (SCI); we hoped to further elucidate the balance of factors involved in cardiovascular regulation to narrow down the mechanisms of impairment in an effort to understand how to potentially reverse it.

Pulmonary and cardiovascular dysfunction are secondary to $\mathrm{SCl}$, with dysfunction increasing as the lesion ascends rostrally due to the increasing impairment of respiratory motor and sympathetic neurons that emerge from the spinal cord. However, there is additional dysfunction that is exacerbated by the injury itself due to chronic immobility (Wecht et al 2003). Pulmonary dysfunction arises due to compensation of intact respiratory muscles for those caudal to the lesion, which further impairs cardiovascular function. Our second aim was to use 
a variety of tests to evaluate cardiovascular regulation in order to better understand the mechanisms involved. Therefore, we hypothesized that persons with SCI will have significantly impaired respiratory motor control and cardiovascular regulation when compared to non-injured (NI) persons, irrespective of level and completeness of injury.

\section{METHODS}

We evaluated respiratory motor function and cardiovascular regulation using a Respiratory Motor Control Assessment (RMCA, 42) and Orthostatic Stress Test (Orthostatic Stress Test, 47). Spirometric outcomes and airway pressure generation evaluated pulmonary function during the RMCA, while a sustained maximum expiratory pressure (5s MEP) maneuver assessed baroreceptor sensitivity (BRS; Evaluation of Baroreceptor Sensitivity, 54). The Orthostatic stress test examined balance of autonomic tone, and cardiovascular regulation using the baroreceptor sequencing method. Outcomes of SCI persons ( $\mathrm{n}=47$; see Table III-2 and Table III-3) were compared to $11 \mathrm{NI}$ persons using a univariate linear regression (model 1-A, Table III-4). Values are reported as mean \pm standard deviation

\section{RESULTS}

Both pulmonary function outcomes and cardiovascular outcomes were significantly decreased in the $\mathrm{SCl}$ group when compared to $\mathrm{NI}$ : airway pressure 
and spirometric outcomes were significantly decreased (Figure V-1), and persons with $\mathrm{SCl}$ demonstrated a significantly impaired response to cardiovascular stress. Persons with $\mathrm{SCl}$ had significant deficits in sympathetic engagement while upright (Figure V-2), and decreased, albeit insignificantly, vagal activity while upright Figure V-3), indicating that our SCI participants attempted to maintain blood pressure (BP) during the Orthostatic Stress Test by vagal inhibition. This caused a significant decrease in R-R interval (RRI) while upright (Figure V-4), though that was not enough to prevent significant drops in systolic BP (SBP; Figure V-5) and diastolic BP (DBP; Figure V-6).

We also found that the cardiovascular regulatory mechanisms that respond to orthostatic stress were significantly impaired. There were fewer significant (i.e., engaged reflexes) up-up (Figure V-7) and down-down (Figure V-9) ramps identified by the BRS sequencing method, indicating that at rest and during stress, the reflexes that coordinate heart rate and vasomotor tone in response to increases (Figure V-7) and decreases (Figure V-9) in BP are significantly impaired. Decreased significant up-up and down-down ramps also resulted in a significantly decreased baroreceptor effectiveness indices (BEI; Figure V-8 and Figure V-10) while upright, or the ratio of significant ramps to nonsignificant ramps. The slope (in $\mathrm{ms} / \mathrm{mmHg}$ ) of significant BRS ramps were measured, but were not found to be significantly different than $\mathrm{NI}$ slopes. 
Finally, BRS outcomes from the 5s MEP maneuver demonstrated significant impairments to sympathetic engagement (Figure V-11) and an insignificantly decreased vagal response (Figure V-12).

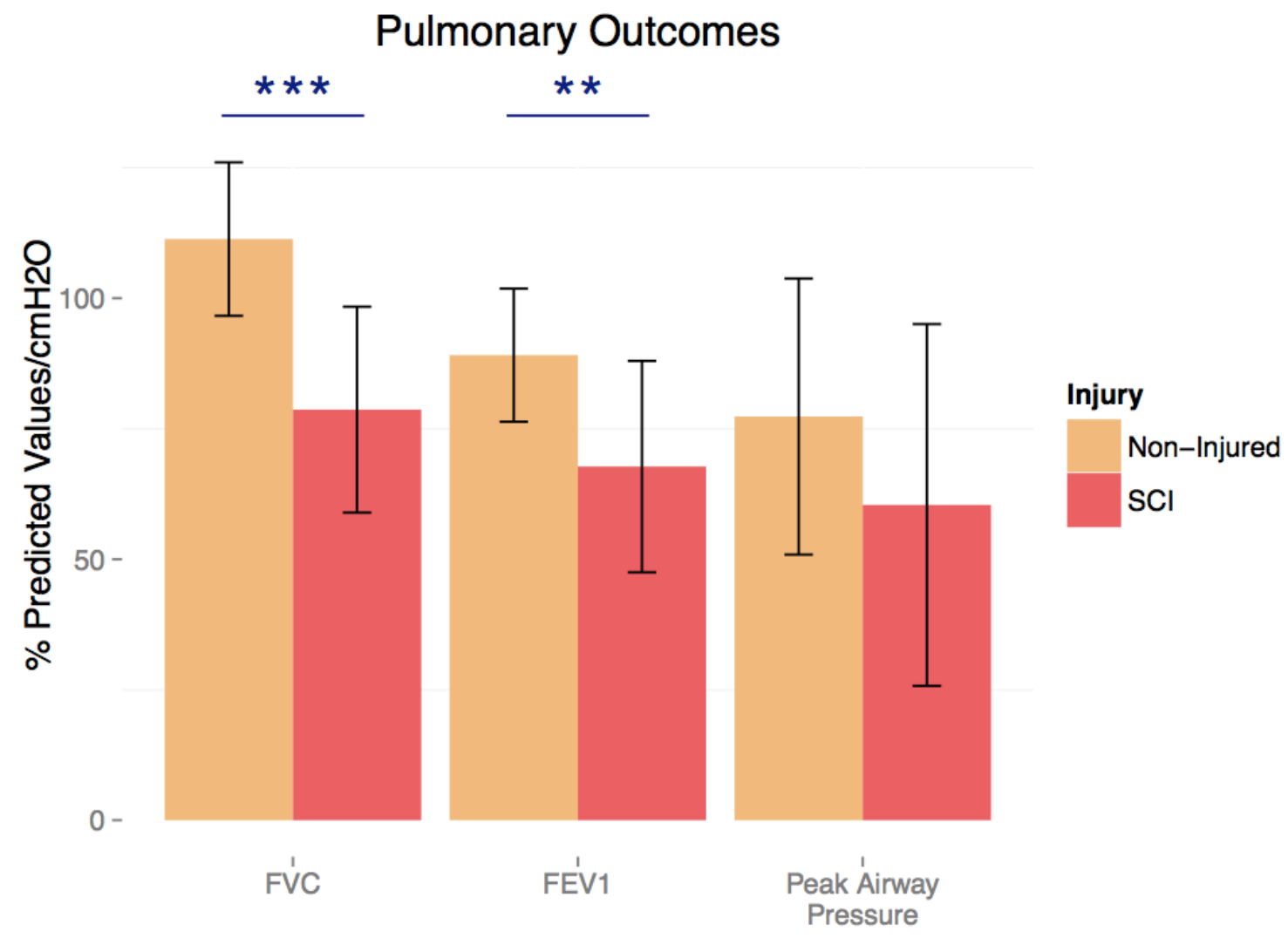

Figure V-1: Pulmonary Function Outcomes in SCl and NI Persons Model 1-A found that the percent predicted values of forced vital capacity (FVC) and forced expiratory volume in $1 \mathrm{~s}\left(\mathrm{FEV}_{1}\right)$ are significantly decreased when comparing non-injured (NI) to spinal-cord injured (SCl) values. FVC: $111 \pm 15 \%$ vs $81 \pm 19 \% ; p<.001$. FEV $_{1}: 91 \pm 13 \%$ vs $69 \pm 21 \% ; p=.004$. MEP: $77 \pm 26$ $\mathrm{cmH}_{2} \mathrm{O}$ vs $60 \pm 35 \mathrm{cmH}_{2} \mathrm{O} ; \mathrm{p}=.181$. Pulmonary function is thus significantly decreased in the $\mathrm{SCl}$ population compared to the NI population, indicating a significant deficit in respiratory motor control irrespective of level or completeness 
of injury. $\mathrm{NI} n=11 ; \mathrm{SCI} n=47$; values are represented as mean \pm standard deviation. 


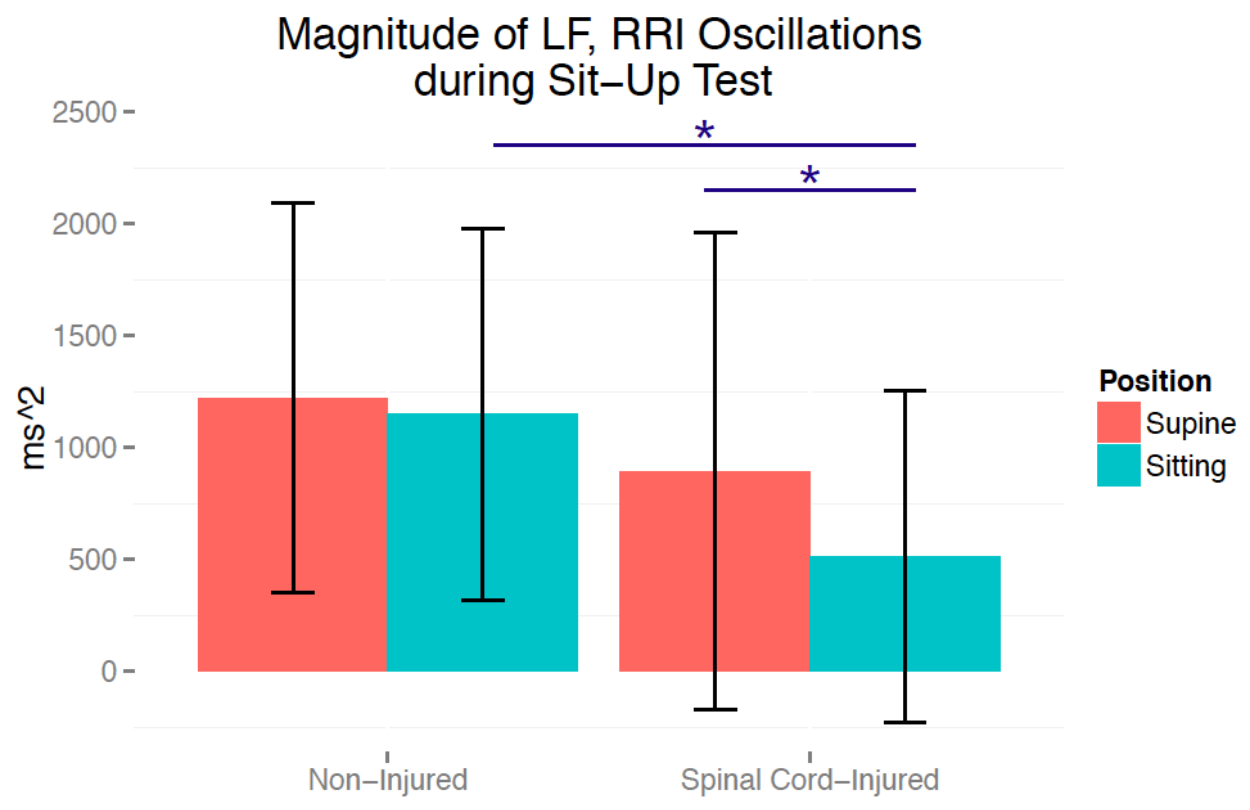

Figure V-2: Sympathetic Heart Rate Variability in SCl and NI Persons Model 1-A found that the power of Low-Frequency (LF) RRI oscillations while seated was significantly lower $(p=.03)$ in the spinal-cord injured $(\mathrm{SCl})$ group $\left(512 \pm 609 \mathrm{~ms}^{2}\right)$ than non-injured (NI) group $\left(1148 \pm 831 \mathrm{~ms}^{2}\right)$. Additionally, the change in RRI power was significantly worse (model $5, \mathrm{p}=.02$ ) in the $\mathrm{SCl}$ group compared to $\mathrm{NI}$ ( $\mathrm{Nl}: 99 \pm 733 \mathrm{~ms}^{2}$ vs SCl: $-472 \pm 1076 \mathrm{~ms}^{2}$ ). While supine, LF power is a result of the combined activity of both the PNS and SNS. LF power while seated, however, illustrates the activity of the sympathetic nervous system as it attempts to maintain blood pressure against gravity. The $\mathrm{SCl}$ group, therefore, demonstrated significantly decreased SNS activity and a poor stress response while upright. $\mathrm{NI} n=11 ; \mathrm{SCI} n=47$; values are represented as mean \pm standard deviation. 


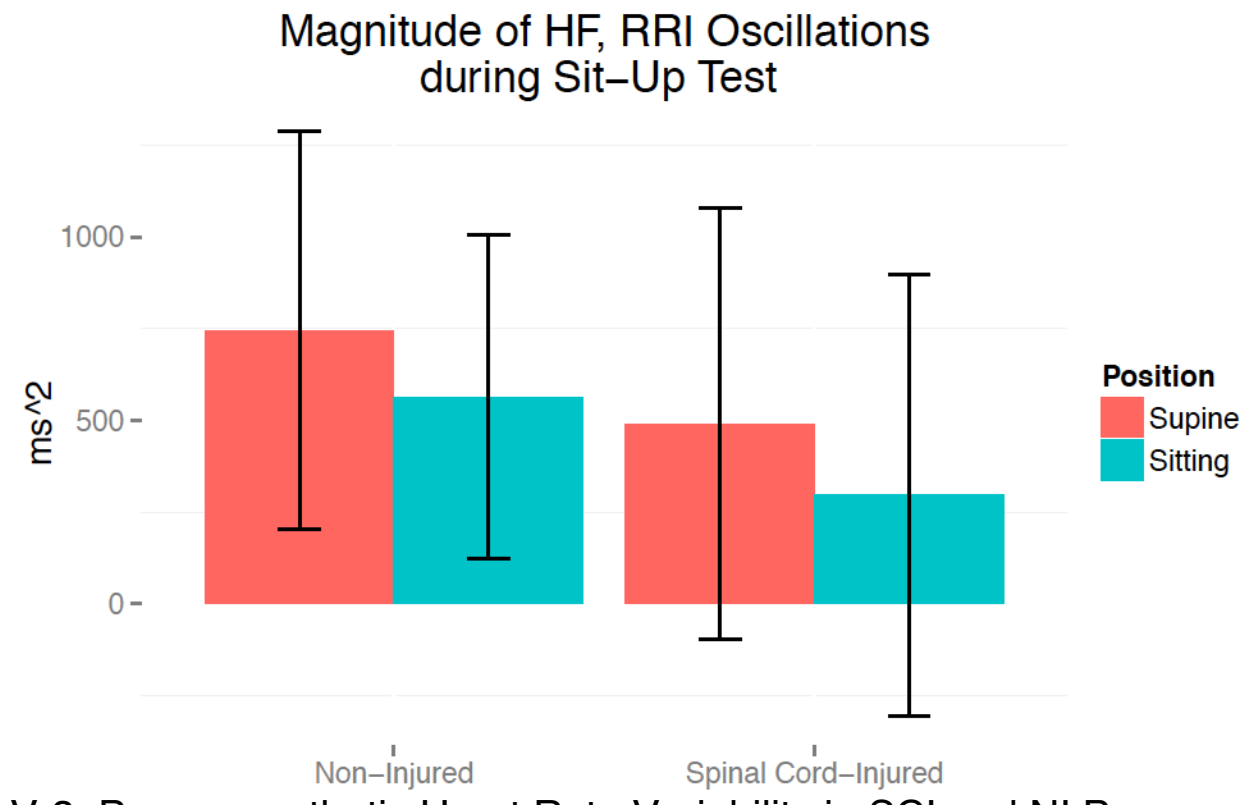

Figure V-3: Parasympathetic Heart Rate Variability in SCI and NI Persons

There were no significant differences found when comparing High-Frequency

(HF), RRI power of spinal-cord injured (SCl) group to that of non-injured (NI)

group, despite a decrease in seated SCl power compared to NI. Parasympathetic activity in persons with $\mathrm{SCl}$ is therefore similar to $\mathrm{NI}$ persons. $\mathrm{NI} n=11 ; \mathrm{SCI} n=$ 47 ; values are represented as mean \pm standard deviation. 


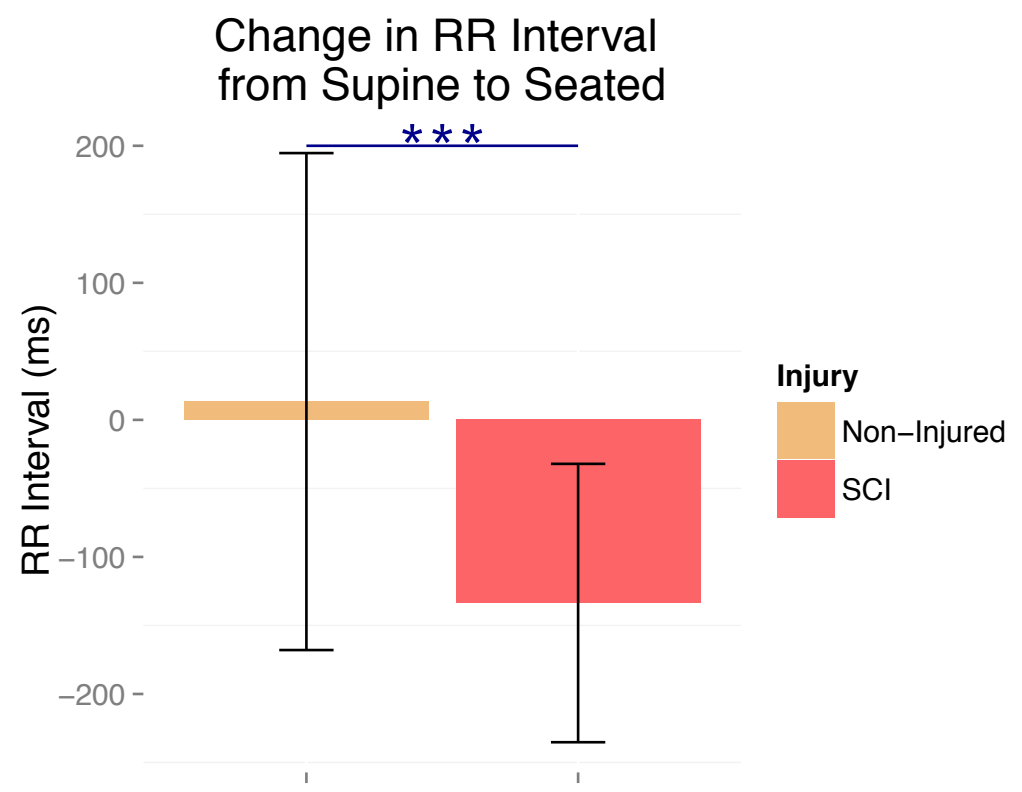

Figure V-4: Change in RRI from Supine to Seated in SCl and NI Persons The drop in RR-Interval while seated was significantly greater (model 5-A, $p<$ $.001)$ in the spinal-cord injured (SCl) $(-133 \pm 101 \mathrm{~ms})$ group when compared to non-injured (NI) (13 $\pm 181 \mathrm{~ms})$. The SCI group, while upright, had a lower heart rate than the $\mathrm{NI}$ group, and demonstrated a poorer regulatory response to orthostatic stress. $\mathrm{NI} n=11 ; \mathrm{SCI} n=47$; values are represented as mean \pm standard deviation. 


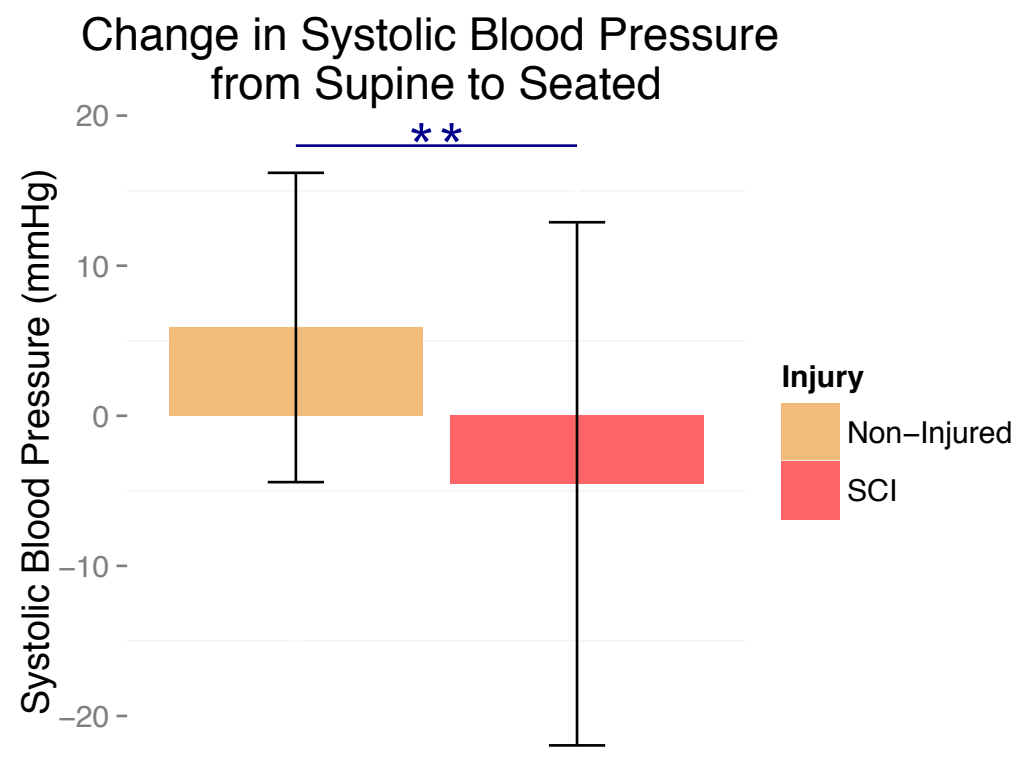

Figure V-5 Change in SBP from Supine to Seated in SCl and NI Persons Drop in systolic blood pressure while upright was significantly greater (model 5-A, $\mathrm{p}=.003)$ in the spinal-cord injured $(\mathrm{SCl})$ group than the non-injured $(\mathrm{NI})$ group: -4 $\pm 17 \mathrm{mmHg}$ vs. $6 \pm 10 \mathrm{mmHg}$, respectively. The SCl group, due to impaired reflex responses, experienced greater pooling of blood in the extremities and was not able to maintain blood pressure against gravity. $\mathrm{NI} n=11$; $\mathrm{SCI} n=47$; values are represented as mean \pm standard deviation. 
Change in Diastolic Blood Pressure

from Supine to Seated

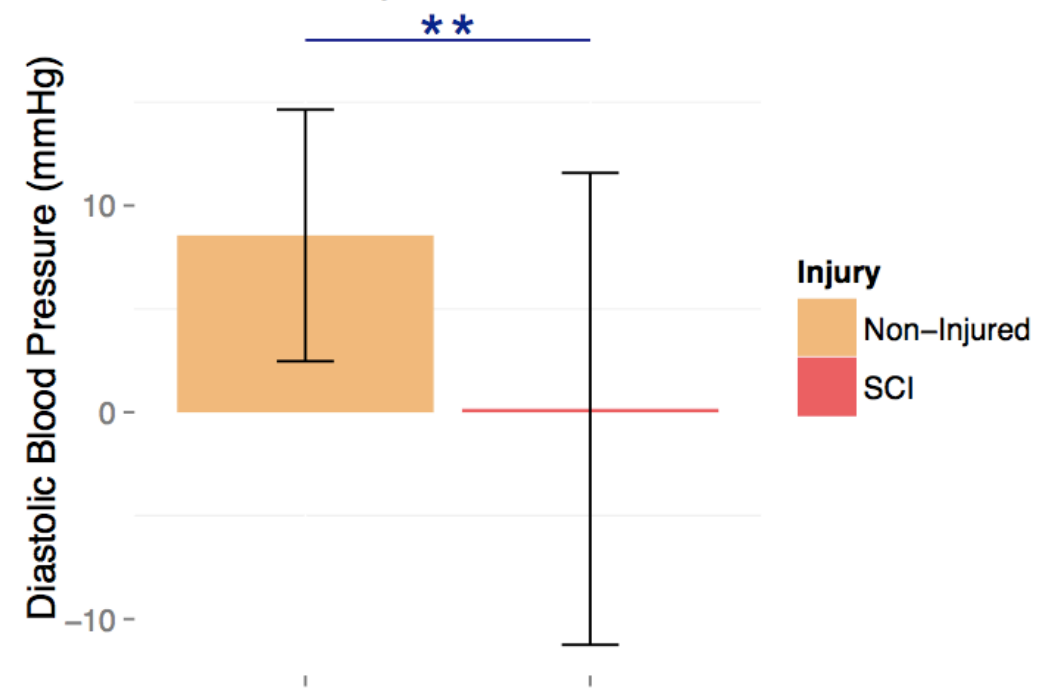

Figure V-6: Change in DBP from Supine to Seated in SCI and NI Persons The change in diastolic blood pressure (DBP) while upright was significantly different (model 1-A, $\mathrm{p}=.003$ ) in the spinal-cord injured (SCI) group compared to non-injured (NI) group: $0.17 \pm 11 \mathrm{mmHg}$ vs. $9 \pm 6 \mathrm{mmHg}$. The change in DBP is typically due to reflex vasoconstriction in order to prevent pooling of blood in the legs and viscera. The impaired reflex responses of the SCI group impaired vasoconstriction and prevented an increase in DBP while upright. $\mathrm{NI} n=11 ; \mathrm{SCl}$ $\mathrm{n}=47$; values are represented as mean \pm standard deviation. 


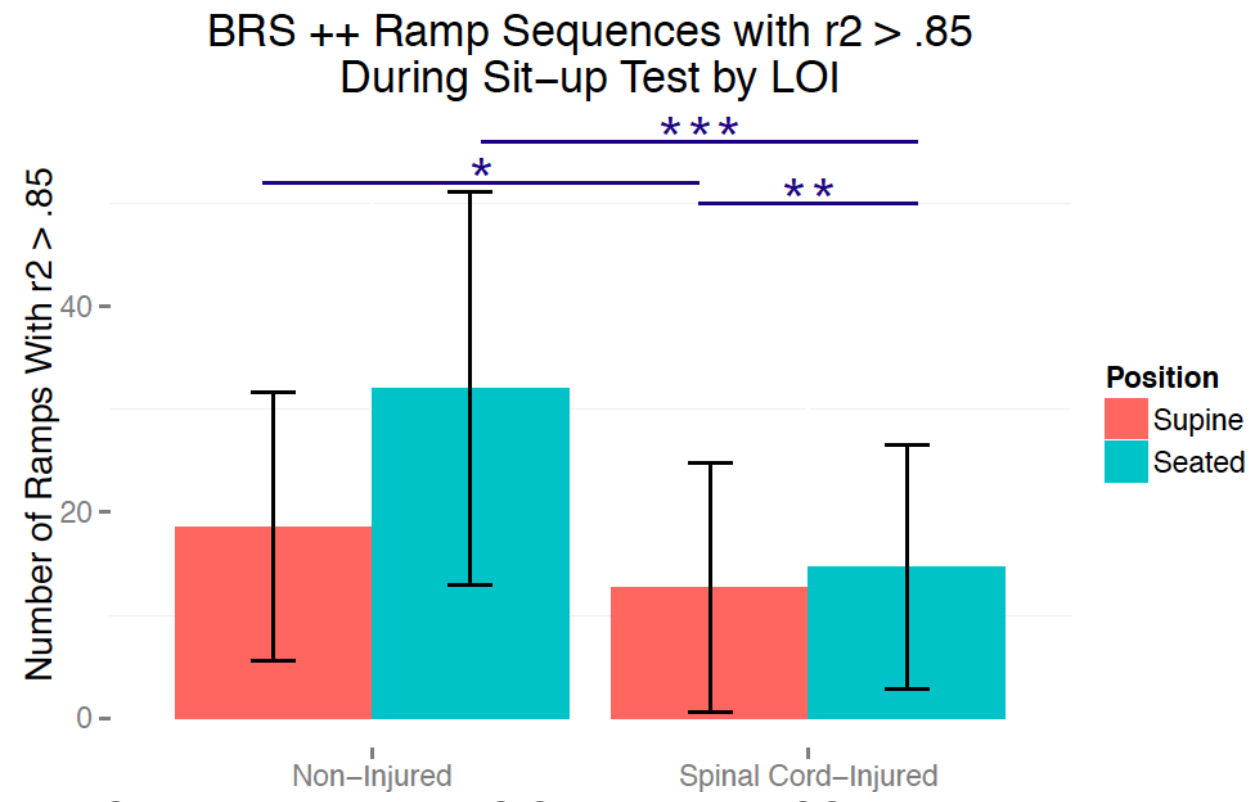

Figure V-7: Significant Up-up BRS Sequences in SCI and NI Persons

The number of significant supine and seated up-up BRS ramp sequences was significantly lower in the spinal-cord injured (SCl) group when compared to the non-injured (NI) group. Supine, the SCl group had $13 \pm 12$ ramps, compared to the NI group that had $19 \pm 13$ ramps (model $5-\mathrm{A}, \mathrm{p}=.04$ ). Sitting, the $\mathrm{SCl}$ group had $15 \pm 12$ ramps, while the NI group had $32 \pm 19$ ramps (model $1-\mathrm{A}, \mathrm{p}=.001$ ) an increase from supine of $2 \pm 12$ and $13 \pm 13$ ramps, respectively (model $1-\mathrm{A}, \mathrm{p}$ $=.01)$. The ++ ramp sequences are a measurement of the ability of the parasympathetic nervous system (PNS) to decrease heart rate and vasoconstriction in response to increases in blood pressure. While supine and sitting the number of ramp sequences were significantly decreased, as was the difference between supine and sitting values. This indicates decreased activity of the PNS in SCl despite all of the participant's injuries sparing the vagus nerve. $\mathrm{NI} n=11 ; \mathrm{SCI} n=47$; values are represented as mean \pm standard deviation. 


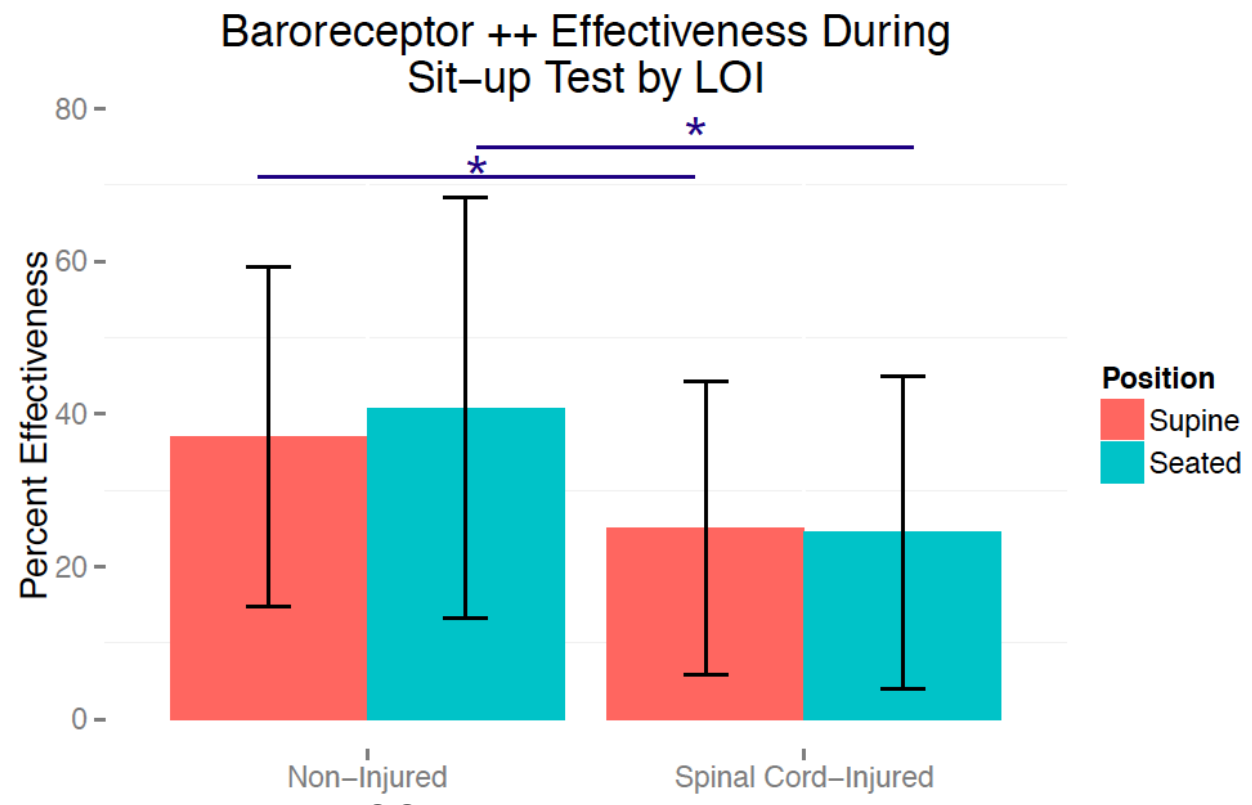

Figure V-8 Up-up BEI in SCl and NI Persons

Up-up baroreceptor effectiveness index (BEI) of the spinal-cord injured (SCI) group was significantly decreased in both the supine (model $5-\mathrm{A}, \mathrm{p}=.04$ ) and sitting (model 5-A, $\mathrm{p}=.03$ ) positions when compared to non-injured $(\mathrm{NI})$. Supine, BEl of SCl group was $25 \pm 19 \%$, compared to the NI BEl of $37 \pm 22 \%$. Sitting, SCI BEI was $25 \pm 20 \%$, while the BEI of the NI group was $40 \pm 27 \%$. This indicates that only one in four ramps of increased systolic blood pressure elicited an increase in $\mathrm{RR}$ interval in the $\mathrm{SCl}$ group, demonstrating a decreased effectiveness of the parasympathetic nervous system to regulate BP when compared to the non-injured group. This decreased parasympathetic activity is despite all participants in the $\mathrm{SCl}$ group having an injury that spared the vagus nerve. $\mathrm{NI} \mathrm{n}=11 ; \mathrm{SCI} \mathrm{n}=47$; values are represented as mean \pm standard deviation. 


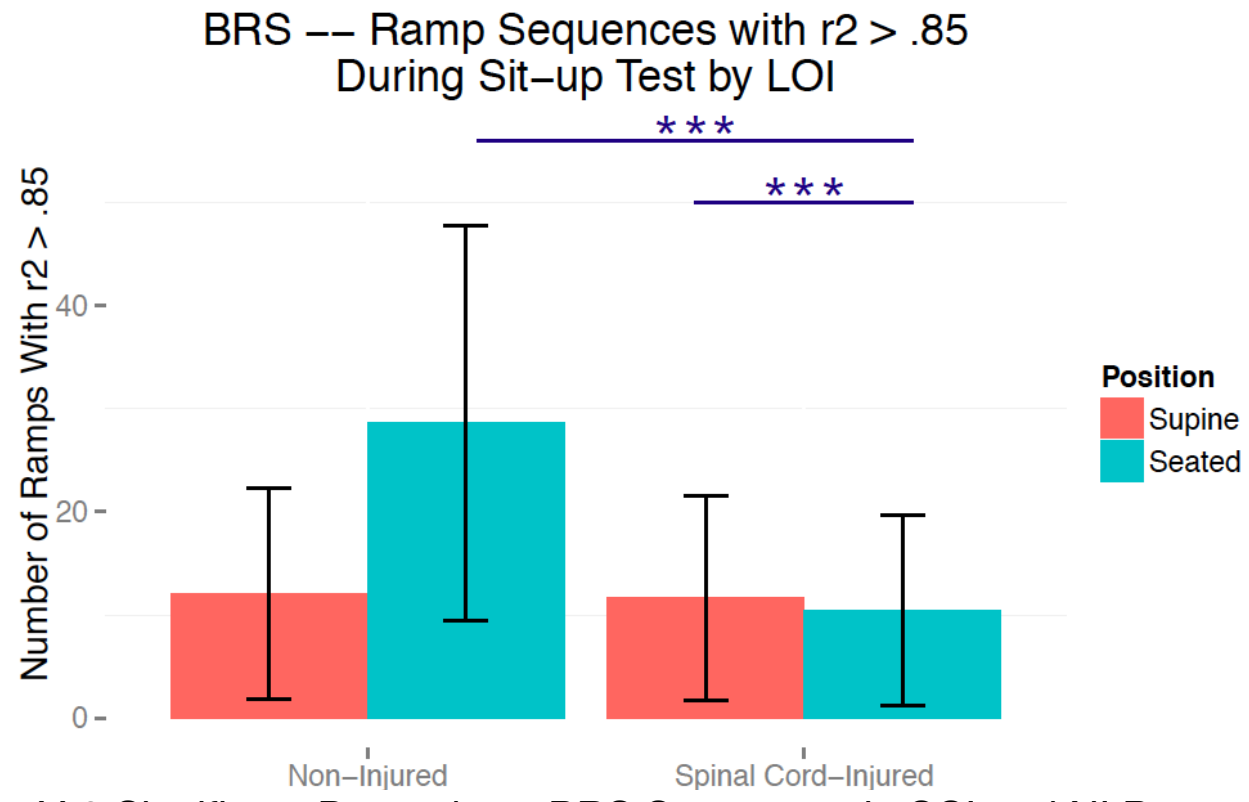

Figure V-9 Significant Down-down BRS Sequences in SCl and NI Persons

The spinal-cord injured (SCl) group had significantly fewer significant down-down (down-down*) BRS sequences while seated compared to the non-injured ( $\mathrm{NI})$ group (model 1-A, $\mathrm{p}<.001$ ) and was significantly different from the supine values when compared to the NI group (model $1-\mathrm{A}, \mathrm{p}<.001$ ). Supine, both the SCI group and the $\mathrm{NI}$ group had $12 \pm 10$ down-down* BRS ramp sequences. Seated, however, the $\mathrm{SCI}$ group had $10 \pm 9$ sequences, while the $\mathrm{NI}$ group had $29 \pm 19$ sequences. Down-down ramp sequences reflect the ability of the sympathetic nervous system to increase heart rate and vasoconstriction in response to drops in blood pressure. The sympathetic stress response in the $\mathrm{SCl}$ group was therefore significantly impaired when compared to the sitting values of the $\mathrm{NI}$ group. $\mathrm{NI} n=11 ; \mathrm{SCI} n=47$; values are represented as mean \pm standard deviation. 


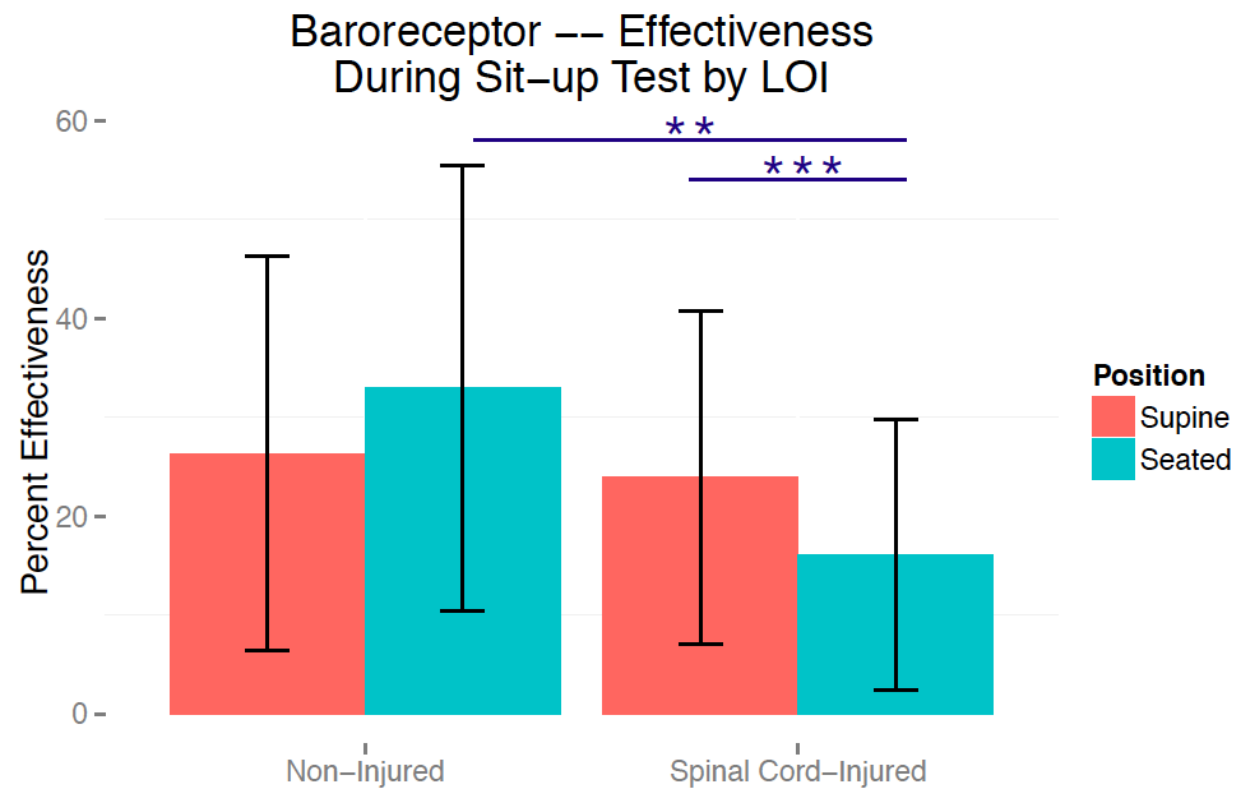

Figure V-10 Down-down BEI in SCl and NI Persons

Down-down baroreceptor effectiveness index (BEI) was significantly decreased (model 1-A, $\mathrm{p}=.007$ ) in the sitting position when comparing the spinal-cord injured (SCl) group to non-injured ( $\mathrm{NI})$, and significantly different from supine (model 5-A, $p=.001$ ). Supine, the SCI down-down BEI was $24 \pm 17 \%$, compared to $26 \pm 20 \%$ in the NI group. Sitting, the SCI down-down BEI fell to 16 $\pm 14 \%$ while the $\mathrm{NI}$ group increased to $33 \pm 22 \%$. Effectiveness is a measurement of the number of significant ramps that elicit a change in heart rate compared to the number of systolic blood pressure ramps overall; it is therefore an indication of the ability of the sympathetic nervous system to regulate heart rate and vasomotor tone in response to drops in blood pressure. The $\mathrm{SCl}$ group demonstrated a significant decrease in BEI during orthostatic stress and thus a significant impairment of the sympathetic reflex response to decreased blood 
pressure. $\mathrm{NI} n=11 ; \mathrm{SCl} n=47$; values are represented as mean \pm standard deviation. 


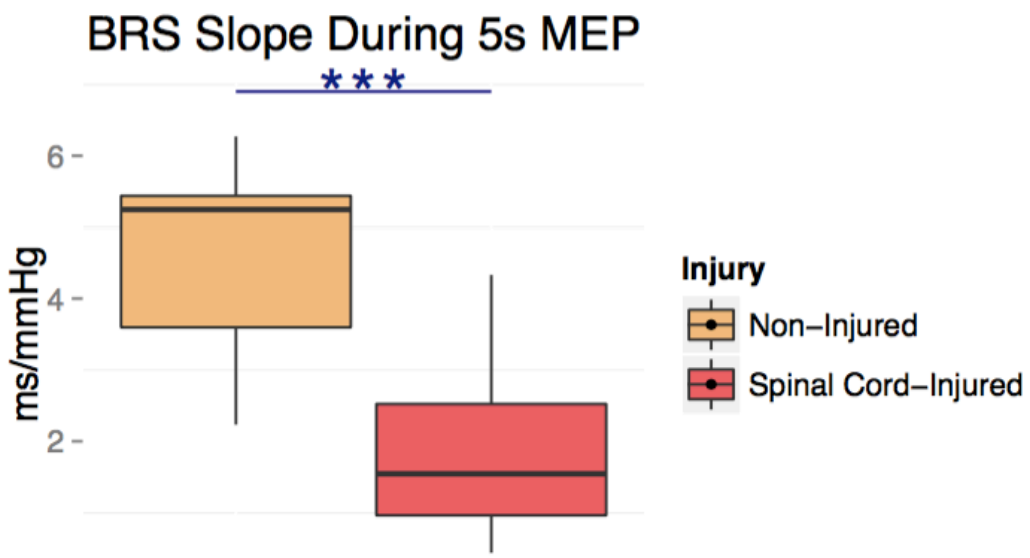

Figure V-11: BRS Slope During 5s MEP in SCl and NI Persons The spinal-cord injured (SCl) group had a significantly decreased baroreceptor sensitivity (BRS) response during the 5s MEP maneuver (called "phase II" because of its relation to the Valsalva maneuver) when compared to the $\mathrm{NI}$ group: $2 \pm 1 \mathrm{~ms} / \mathrm{mmHg}$ compared to $5 \pm 1 \mathrm{~ms} / \mathrm{mmHg}$ (model $1-\mathrm{A}, \mathrm{p}<.001)$. The phase II slope measures the ability of the SNS to increase HR in response to a sustained drop in BP. The participants with $\mathrm{SCl}$ demonstrated a significantly worse sympathetic response than the $\mathrm{NI}$ group, illustrating an impairment of the sympathetic arm of the baroreflex arc. $\mathrm{NI} n=11 ; \mathrm{SCI} n=47$; values are represented as mean \pm standard deviation. 


\section{BRS Slope Following 5s MEP}

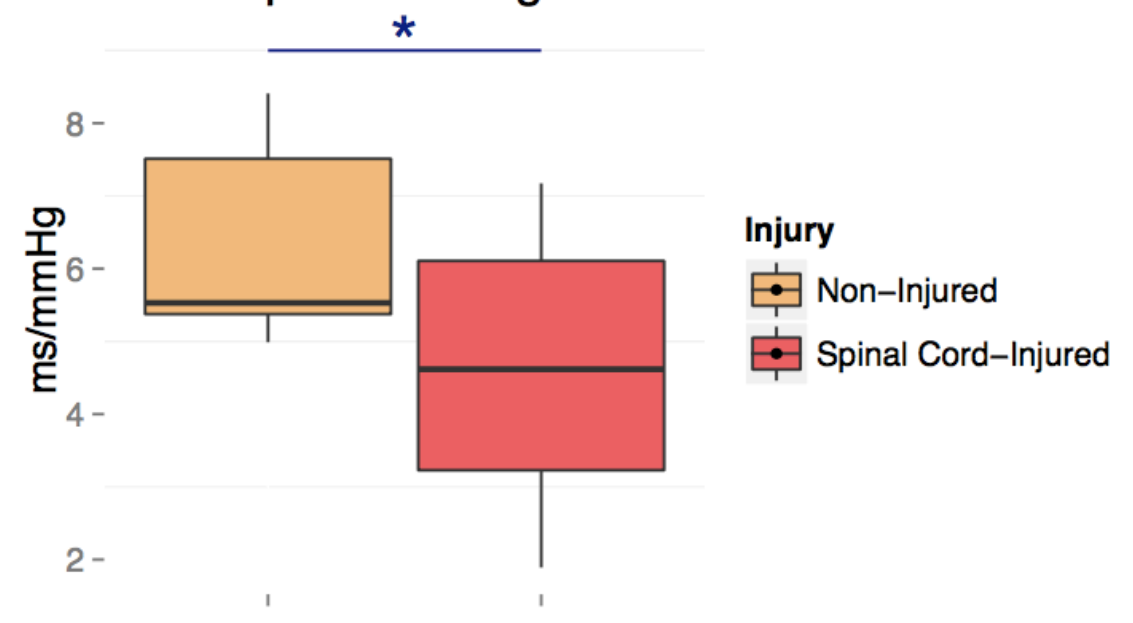

Figure V-12: BRS Slope Following 5s MEP in SCI and NI Persons

The baroreceptor sensitivity (BRS) slope of the recovery period following the $5 \mathrm{~s}$ MEP maneuver (called "phase IV" because of its relation to the Valsalva Maneuver) was significantly decreased in the spinal-cord injured (SCI) group. SCl group had a slope of $5 \pm 2 \mathrm{~ms} / \mathrm{mmHg}$, while the $\mathrm{NI}$ group had a slope of $7 \pm 2$ $\mathrm{ms} / \mathrm{mmHg}$ (model $1-\mathrm{A}, \mathrm{p}=.02$ ). The phase IV slope assesses the cardiovagal loop of the baroreflex. The decreased slope demonstrates decreased reflex response to increases in blood pressure. $\mathrm{NI} n=11 ; \mathrm{SCI} n=47$; values are represented as mean \pm standard deviation. 


\section{DISCUSSION}

We found our first hypothesis to be correct and, without controlling for injury, there were still significant deficits to pulmonary and cardiovascular function in persons with $\mathrm{SCl}$ when compared to NI. Significantly decreased pulmonary function outcomes indicate significant impairments to respiratory motor control in persons with $\mathrm{SCl}$. Our cardiovascular outcomes imply that some of the impairment can be attributed to poor pulmonary function: first, the balance of autonomic tone while upright demonstrates that persons with $\mathrm{SCl}$ attempted to maintain blood pressure during orthostasis by vagal withdrawal, which resulted in a significantly decreased RRI. As such, our participants do not suffer from widespread vagal desensitization common in $\mathrm{SCl}$ (an attempt to balance vagal tone with the impaired sympathetic tone)(Wecht et al 2003). This implies that the impairments to cardiovascular regulation are not entirely attributable to level of injury: if cardiovascular impairments were only dependent on level of injury, only outcomes evaluating sympathetic reflex arcs would be impaired as all of our participants had injuries caudal to the brainstem. We found significant impairment to both sympathetic and parasympathetic reflex arcs, demonstrated by the significant deficiencies in the significant up-up and down-down BRS sequences and BRS outcomes obtained from phases II and IV of the 5s MEP maneuver. Thus there was an impediment to reflex activation, either in receptor engagement or engagement of the efferents by the receptors themselves. Desensitization or inactivity of the receptors that regulate cardiovascular reflexes can be attributed 
to poor pulmonary function due to the close relationship therein: chronic disuse can lead to desensitization of the receptors, or poor respiratory motor control and subsequent decrease in airway pressure generation can decrease the activation of the cardiopulmonary receptors and respiratory pump (Frisbie 2005, van Gestel \& Steier 2010).

Therefore, it is likely that our participants experienced impairments to cardiovascular regulation that were related to pulmonary dysfunction and not just level of injury. In order to prove this irrefutably, we decided to look at these outcomes and examine the role played by level and completeness of injury in our population. If we found that cardiovascular function has little to no relationship with level and completeness of injury that would solidify the role pulmonary dysfunction plays in cardiovascular dysfunction. 


\section{CHAPTER VI:}

\section{CARDIOVASCULAR REGULATION AND LEVEL OF SPINAL CORD INJURY}

\section{INTRODUCTION}

Having demonstrated the relationship between pulmonary and cardiovascular dysfunction in spinal cord injury (SCl; i.e., some of the dysfunction of the cardiovascular system is due to poor reflex activity, which can be attributed to impaired respiratory motor control), our second aim was to evaluate the role played by level and completeness of injury on cardiovascular dysfunction. If we found that cardiovascular dysfunction was not correlated to level or completeness of injury, it would mean that there was an additional confounder in the relationship between cardiovascular function and level and completeness of injury. We hypothesized, therefore, that pulmonary function would correlate significantly with level of injury, but cardiovascular function would not; cardiovascular function would be decreased throughout all levels of injury, demonstrating the deficiencies in cardiovascular regulation caused by pulmonary dysfunction. If hypotheses one and two were correct, we would demonstrate, first, that desensitization of the receptors occurs in SCl due to poor pulmonary 
function, and second, that results in poor cardiovascular regulation across all levels of injury, not just those with impaired sympathetic efferents. It would further confirm that cardiovascular impairment in $\mathrm{SCl}$ is more complex than previously imagined.

\section{METHODS}

We used outcomes from a Respiratory Motor Control Assessment (RMCA, 42) and Orthostatic Stress Test (Orthostatic Stress Test, 47) to evaluate pulmonary function and cardiovascular regulation. Spirometric outcomes, airway pressure generation, heart rate variability (HRV), and baroreceptor sensitivity (BRS) were correlated to level of injury and AIS impairment ( $n=47$; Table III-4, models 2-7A) using univariate and multivariate linear regressions. BRS was analyzed using the 5s MEP (Evaluation of Baroreceptor Sensitivity, 54) and the sequencing method. For the statistical models, injury was either a factor (cervical or thoracic injury) or an interval variable (a rank based on level of injury, from C2 to T12). Values are reported as mean \pm standard deviation.

\section{RESULTS}

There were significant correlations between spirometric outcomes (percent predicted values of forced vital capacity, FVC, and forced expiratory volume in 1 second, $\mathrm{FEV}_{1}$ ), airway pressure generation, and level of injury (Figure VI-1). Significance was not increased when controlling for injury, and there were no 
significant relationships between pulmonary function outcomes and AIS impairment. Respiratory motor control is thus related to level of injury instead of completeness of injury. There was no linear correlation between level of injury and change in RRI, systolic BP, or diastolic BP. Both cervical and thoracic groups decreased RRI in response to orthostasis, cervical participants significantly so; however, the thoracic group did not experience a significant drop in blood pressure while upright. The thoracic group thus was able to recruit the vasculature with heart rate in order to maintain blood pressure. Despite this, thoracic participants still demonstrated impaired autonomic tone and impaired BRS when compared to non-injured persons.

First, there were no linear correlations between sympathetic and parasympathetic activity while upright. Both cervical and thoracic groups were decreased compared to non-injured persons; however, only while upright was the cervical sympathetic power significantly decreased. Vagal power decreased while upright in both non-injured and SCl groups.

Both cervical and thoracic participants experienced fewer significant up-up (up-up*) and significant down-down (down-down*) BRS ramps while upright and when compared to supine. Cervical participants had significantly fewer up-up* and down-down* ramp sequences while supine, as well, and a significantly lower baroreceptor effectiveness index (BEI) in both positions. Additionally, there was no significant linear relationship between BRS ramps and BEI and level of injury, nor a relationship between AIS impairment. The slope (in $\mathrm{ms} / \mathrm{mmHg}$ ) of 
significant BRS ramps were measured, but were not found to be significantly different than $\mathrm{NI}$ slopes, nor were they correlated to level and completeness of injury.

Finally, cervical and thoracic groups had significantly lower BRS responses during the 5s MEP than non-injured participants; the BRS outcome after the 5s MEP was lower than non-injured outcomes in both groups, albeit insignificantly. 


\section{Sitting Pulmonary Outcomes by LOI}

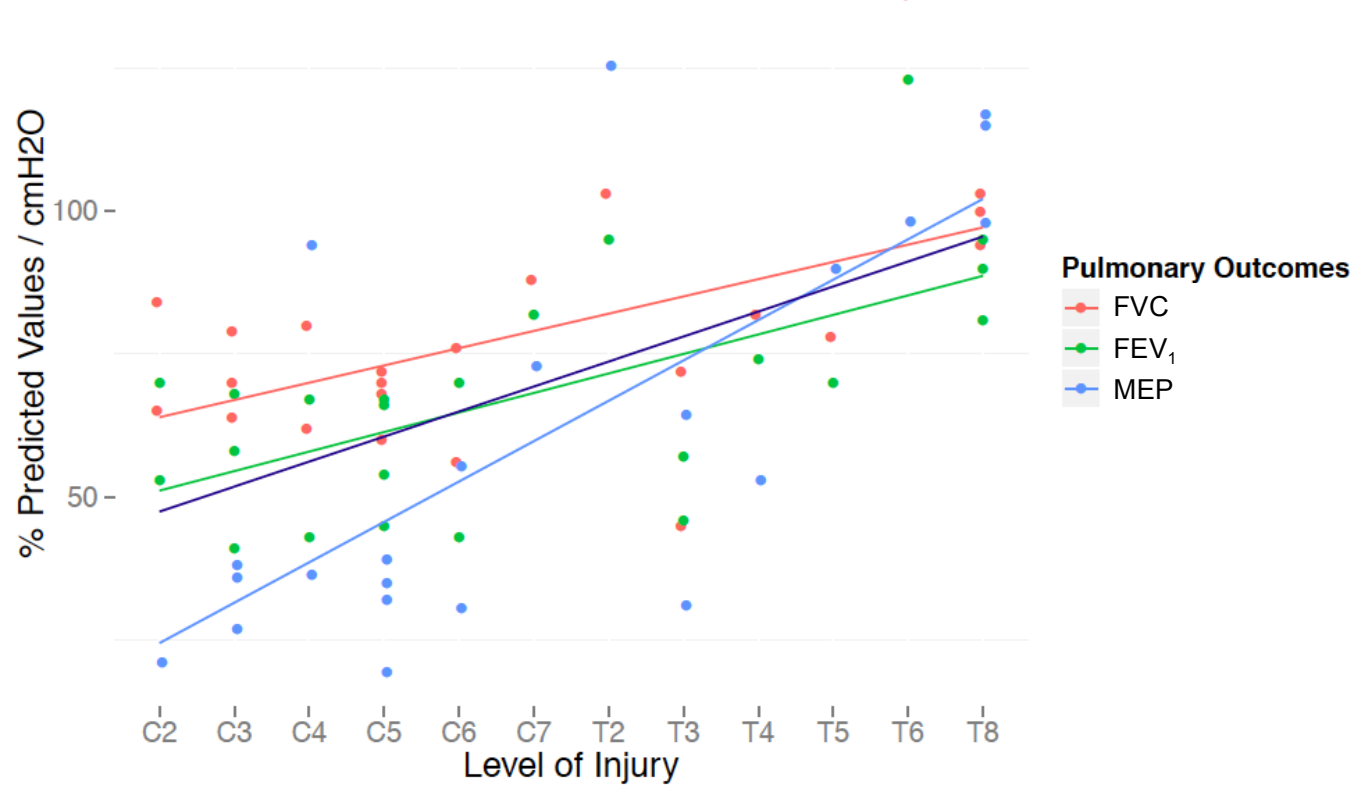

Figure VI-1: Pulmonary Function Outcomes by LOI

Forced vital capacity $(F V C)$, forced expiratory volume in $1 \mathrm{~s}\left(\mathrm{FEV}_{1}\right)$ and maximum expiratory pressure $\left(\mathrm{MEP} ; \mathrm{cmH}_{2} \mathrm{O}\right)$ correlate significantly with level of injury. FVC $\%: p=.01, R^{2}=.82($ model $3-A) ; F^{2} V_{1} \%: p=.001, R^{2}=.77$ (model 3-A); MEP $\mathrm{cmH}_{2} \mathrm{O}: \mathrm{p}<.001, \mathrm{R}^{2}=.72$ (model3-A). The dark blue line is the mean. Model 7A, which controlled for AISA (American Spinal Injury Assessment) impairment score, did not increase the significance of this relationship. This could be due to the lack of specificity the AIS assessment demonstrates with trunk and respiratory motor function. Level of injury in our cohort was therefore a better predictor for pulmonary function outcome. SCI $n=47$. 


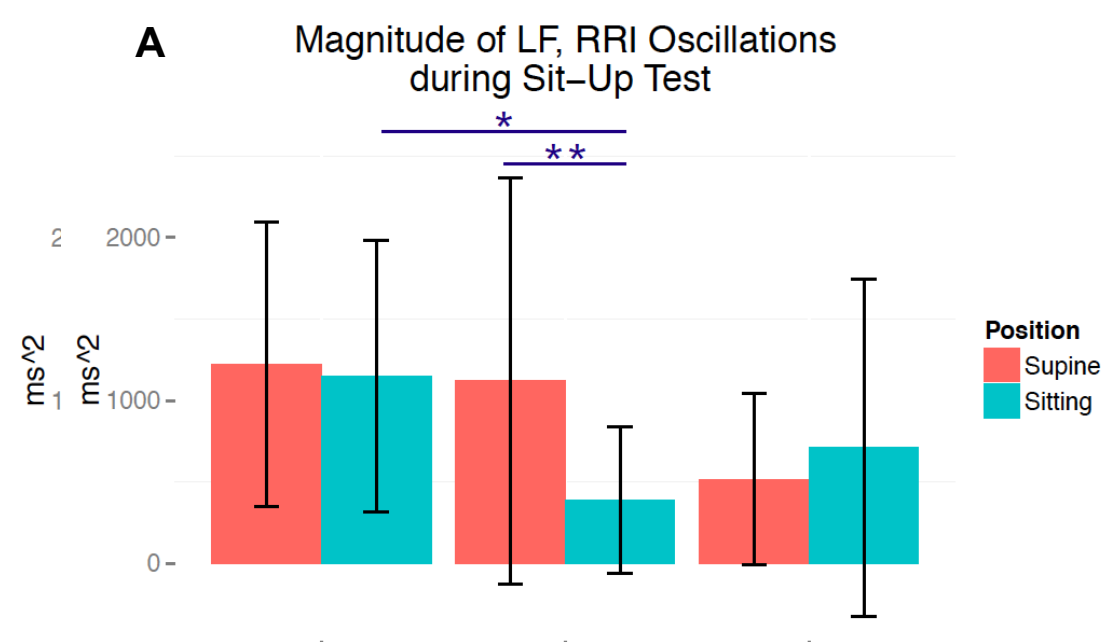

B HRV in the LF Range while Sitting by LOI

Non-Injured

Cervical ScI

Thoracic SCl

$33000-$

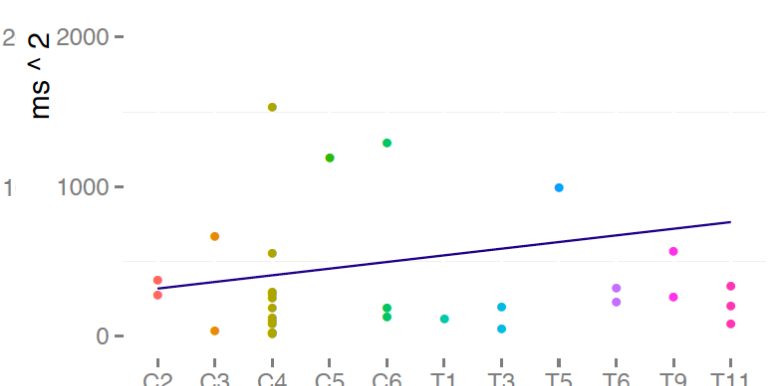

Figure VI-2: Sympathetic Heart Rate Variability Outcomes by LOI

(A) Cervical Low-Frequency (LF) power while supine was $1120 \pm 1247 \mathrm{~ms}^{2}$, compared to $1221 \pm 872 \mathrm{~ms}^{2}$ in the NI group and $516 \pm 527$ in the Thoracic group. Seated, Cervical LF power decreased to $385 \pm 450 \mathrm{~ms}^{2}$ while the thoracic LF power increased to $710 \pm 1036 \mathrm{~ms}^{2}$. The NI power stayed nearly the same at $1149 \pm 832 \mathrm{~ms}^{2}$. There is a significant difference between the Cervical LF, RRI power while seated when compared to the NI group (model 2-A, $p=.02$ ) and the difference between seated and supine values (model $2-A, p=.04$ ). However, there are no differences between the thoracic group sitting values or delta values (model 2-A: $\mathrm{p}=.19$ and $\mathrm{p}=.76$, respectively) compared to the $\mathrm{NI}$ group; (B) neither is there 
a linear relationship between LF RRI power and level of injury (model 7-A: $p=.54, R^{2}=.46$ ). A decreased LF power in the cervical group demonstrates the inability of the sympathetic nervous system (SNS) to respond to stress: while seated, LF power should remain about the same or increase due to increased HR (as seen in the NI and thoracic groups).

However the cervical group demonstrated a significant decrease in LF power and thus SNS control during stress due to the impaired descending SNS neurons. $\mathrm{NI} n=11 ; \mathrm{SCI} n=47$; values are represented as mean \pm standard deviation. 
A Magnitude of HF, RRI Oscillations during Sit-Up Test

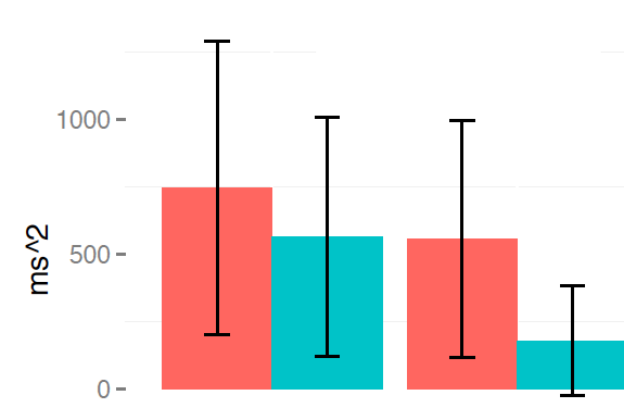

$-500-$

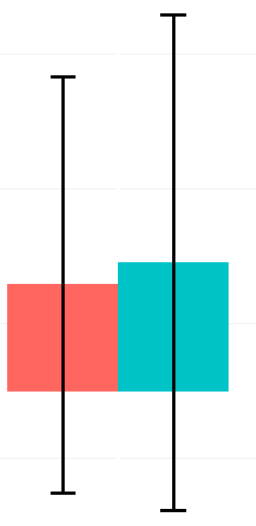

Thoracic SCI
B $\quad$ HRV in the LF Range while Sitting by LOI

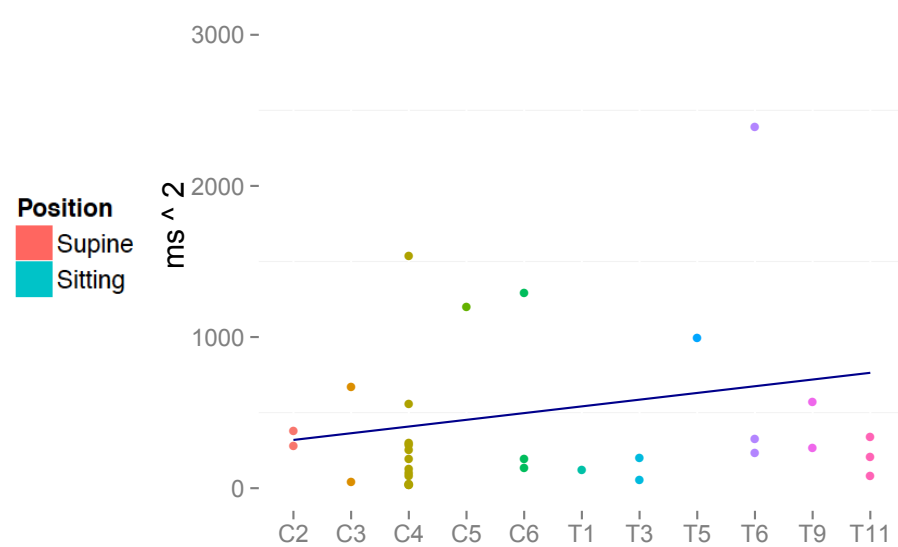

Figure VI-3: Parasympathetic Heart Rate Variability Outcomes by LOI

(A) Supine values of all three groups were not significantly different: non-injured (NI) high-frequency (HF) RRI power was $746 \pm 544 \mathrm{~ms}^{2}$; Cervical HF power was $556 \pm 439 \mathrm{~ms}^{2}$ (model 2-A, $\mathrm{p}=.80$ ); thoracic HF power was $394 \pm 771 \mathrm{~ms}^{2}$ (model 2-A, $p=.11)$. While seated, the cervical HF power decreased (178 \pm 203 ; model 2-A, $p=.09$ ) compared to $\mathrm{NI}$, while the thoracic power was similar $(476 \pm 917$; model 2-A, $p=.72)$. (B) There was no linear relationship between HF power and LOI (model $3-\mathrm{A}: \mathrm{p}=.62, \mathrm{R}^{2}=.35$ ). HF oscillations in $\mathrm{RRI}$ are due to the activity of the parasympathetic 
nervous system and cardiopulmonary receptors that match respiration with heart rate. The pronounced decrease in the cervical group demonstrates the decrease in parasympathetic activity during stress to compensate for the inability of the sympathetic nervous system to increase heart rate. $\mathrm{NI} n=11 ; \mathrm{SCl} n=47$; values are represented as mean \pm standard deviation. 

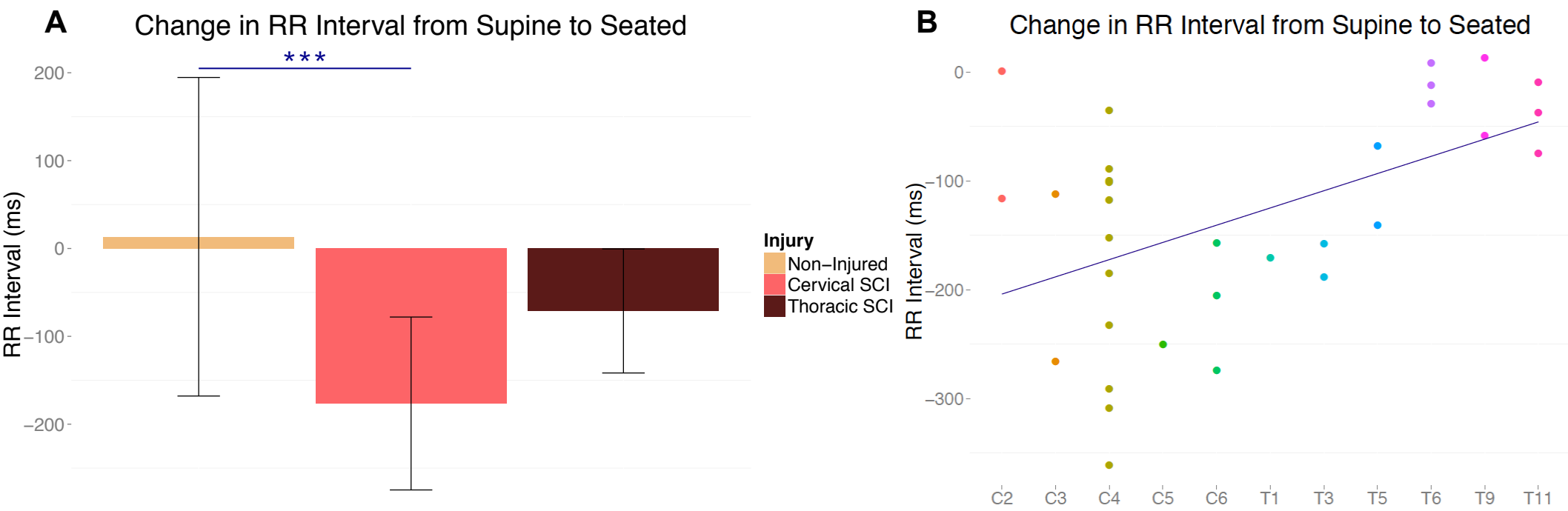

Figure VI-4: Change in RR Interval from Supine to Seated by LOI

(A) Change in RR Interval (RRI) upon assumption of an upright posture was significantly different (model $2-A, p<.001$ ) in the cervical group: in the non-injured group, RRI increased by $13 \pm 181 \mathrm{~ms}$, whereas the cervical group decreased by $176 \pm 98 \mathrm{~ms}$, and the thoracic group decreased by $-71 \pm 71 \mathrm{~ms}$ (model $3-\mathrm{A}, \mathrm{p}=.10$ ). (B) The linear relationship between change in RRI and level of injury was not significant (model $3-A: p=.35, R^{2}=.44$ ). The decrease in RRI is counterintuitive, because the sympathetic nervous system (SNS) is responsible for increasing heart rate during stress. 
However, the cervical group demonstrated a decrease in parasympathetic activity while upright (Figure VI-3) a

compensation for poor SNS activity that would increase HR. Additionally, thoracic injuries have varying degrees of SNS activity, so the increased HR could be a combination of vagal withdrawal and available SNS activation. $\mathrm{NI} n=11 ; \mathrm{SCl} n=$ 47 ; values are represented as mean \pm standard deviation. 
A Change in Systolic Blood Pressure from Supine to Seated

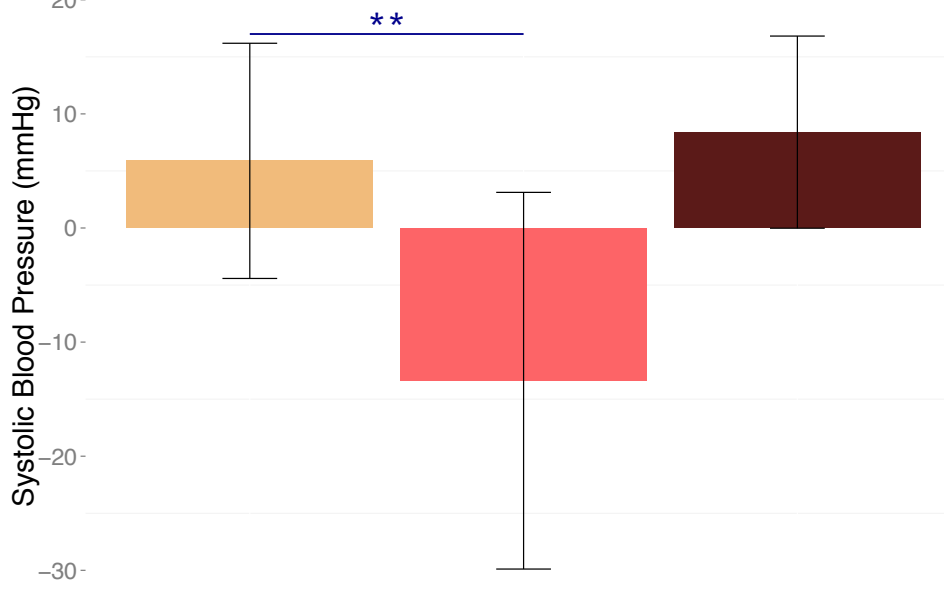

B Change in Systolic Blood Pressure from Supine to Seatec

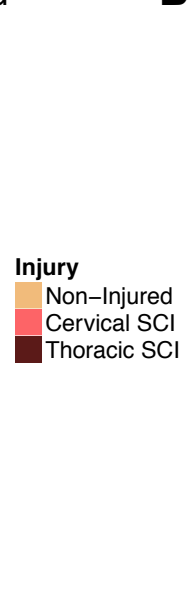

Figure VI-5: Change in SBP from Supine to Seated by LOI

(A) There was a significant drop (model 2-A, $p<.001$ ) in systolic blood pressure $(\mathrm{SBP})$ values while upright in the cervical group compared to the non-injured (NI): NI SBP increased while upright by $6 \pm 10 \mathrm{mmHg}$, while the cervical group decreased by $-13 \pm 17 \mathrm{mmHg}$; there were no significant differences between the thoracic and non-injured groups (SBP increased by $8 \pm 8 \mathrm{mmHg}$, model 2-A $p=.66$ ). (B) Likewise, there was not a significant linear relationship between the change in blood pressure from supine to seated and level of injury (model $3-A: p=.08, R^{2}=.56$ ). Cervical injuries exhibit decreased sympathetic activity during stress, resulting in the inability to increase heart rate and vasomotor tone to 
the degree required to maintain $\mathrm{BP}$ during orthostatic stress. $\mathrm{NI} n=11 ; \mathrm{SCl} n=47$; values are represented as mean \pm standard deviation. 
A Change in Diastolic Blood Pressure from Supine to Seated

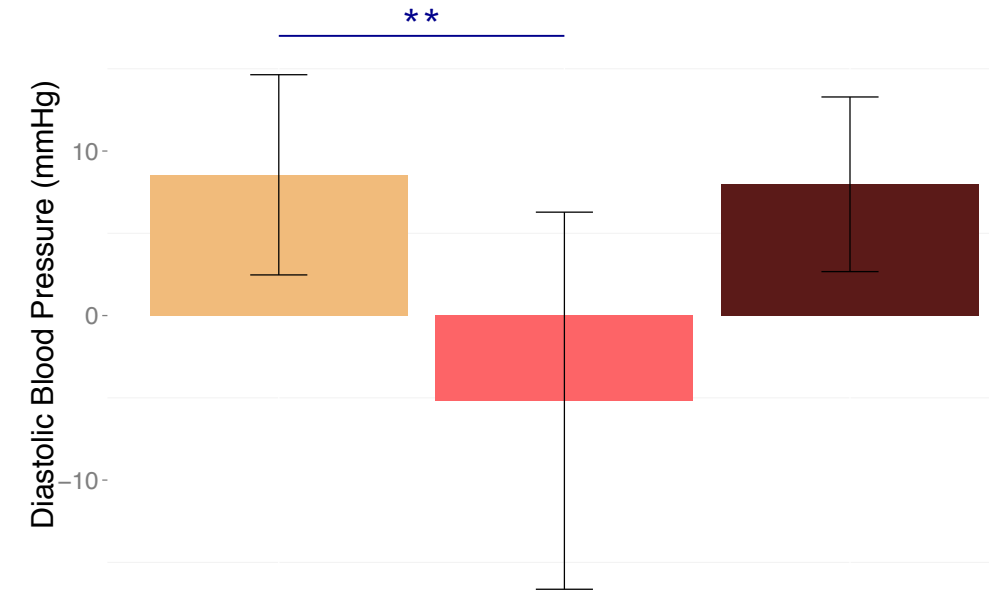

B Change in Diastolic Blood Pressure from Supine to Seated

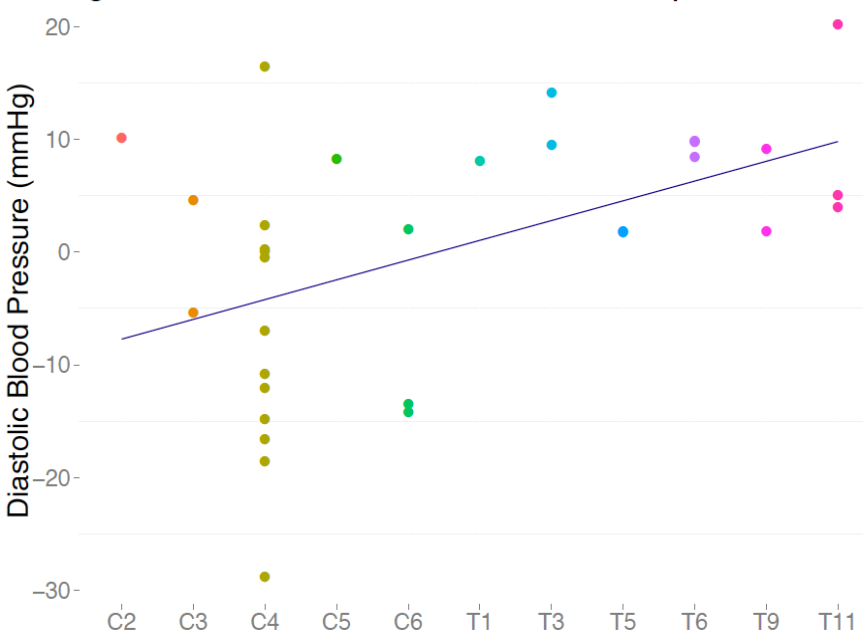

Figure VI-6: Change in DBP from Supine to Seated by LOI

(A) There was a significant drop (model 2-A, $\mathrm{p}<.001$ ) in diastolic blood pressure (DBP) in the cervical group when compared to the non-injured group (NI): NI DBP increased by $9 \pm 6 \mathrm{mmHg}$, while cervical DBP decreased by $-5 \pm 11$ $\mathrm{mmHg}$. Thoracic group DBP increased by $8 \pm 5 \mathrm{mmHg}$ and thus was not significantly different when compared to $\mathrm{NI}$ group (model 2-A, $p=.88$ ). (B) There was no significant linear relationship between the change in DBP from supine to sitting and level of injury (model $3-A, p=.42, R^{2}=.52$ ). The drop in DBP indicates that the cervical group experienced impaired sympathetic activation during stress, and vagal withdrawal (Figure VI-3) alone is not sufficient to sustain DBP 
while upright. Thoracic injuries have varying degrees of sympathetic nervous system activation depending on the location of the injury and evidently that is sufficient to prevent drops in DBP while upright. $\mathrm{NI} n=11 ; \mathrm{SCI} n=47$; values are represented as mean \pm standard deviation. 

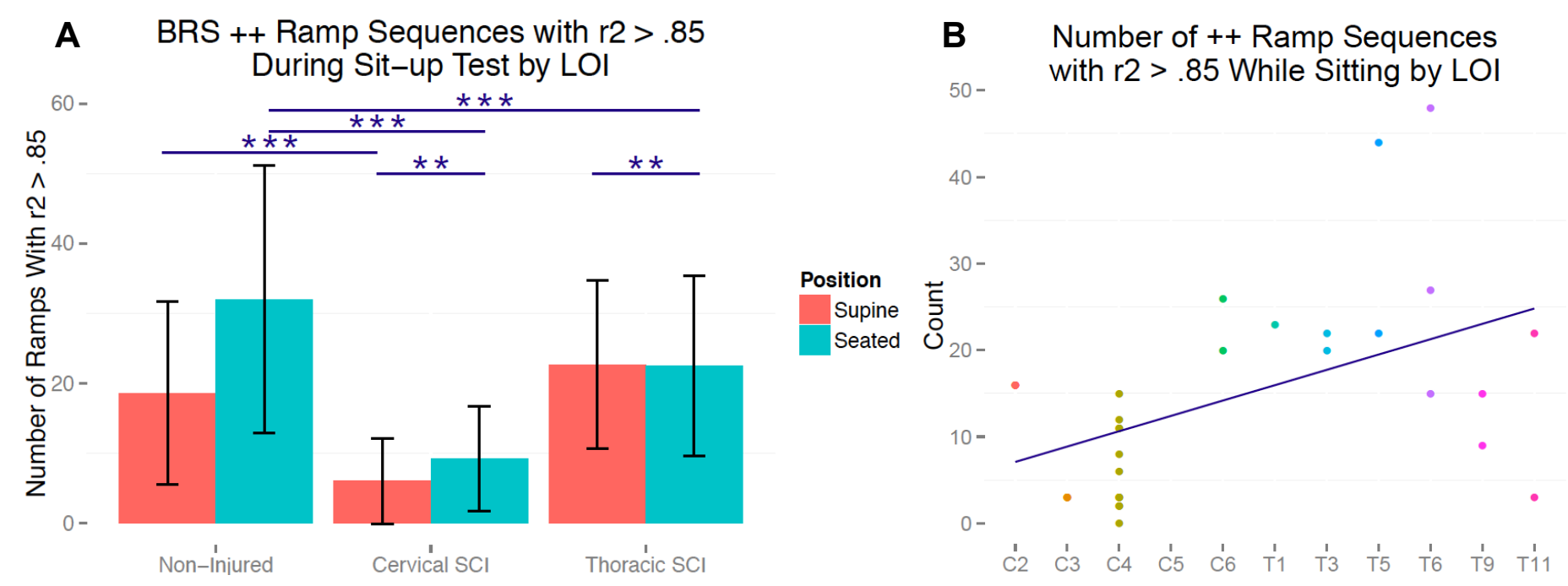

Figure VI-7: Significant Up-up BRS Sequences by LOI

(A) There were significant differences between the number of significant up-up (up-up*) ramp sequences when comparing the cervical and thoracic groups to non-injured (NI). The NI group had $19 \pm 13$ up-up* ramps while supine, which increased to $32 \pm 19$ up-up* ramps while seated, an increase of $13 \pm 13$ ramps. The cervical group had $6 \pm 6$ upup* ramps while supine (model 6-A, $p=.001$ ) and $9 \pm 7$ up-up* ramps while seated (model $6-\mathrm{A}, \mathrm{p}<.001$ ), an increase of $3 \pm 10$ ramps (model $2-A, p=.01$ ). The thoracic group had $23 \pm 12$ up-up* ramps while supine (model $6, p=.72$ ) and 22 \pm 13 up-up* ramps while seated (model $2-A, p<.001$ ), a decrease of $0 \pm 13$ ramps (model $1-A, p=.01$ ). (B) There was 
not a significant relationship between the number of significant ramp sequences and level of injury (model 3-A: $p=.83$, $\left.\mathrm{R}^{2}=.52\right)$ despite the visible trend. Significant ++ ramp sequences are a measurement of the body's ability to decrease heart rate and vasoconstriction in response to increases in blood pressure, specifically those sequences with an $R^{2}$ coefficient (derived from SBP and RRI) $>.85$. The cervical group was significantly decreased at baseline and during stress, and the thoracic group was significantly decreased during stress, indicating an impairment of the vagal arm of blood pressure regulation despite injuries that spare the vagus. $\mathrm{NI} n=11 ; \mathrm{SCI} n=47$; values are represented as mean \pm standard deviation. 
A Baroreceptor ++ Effectiveness During

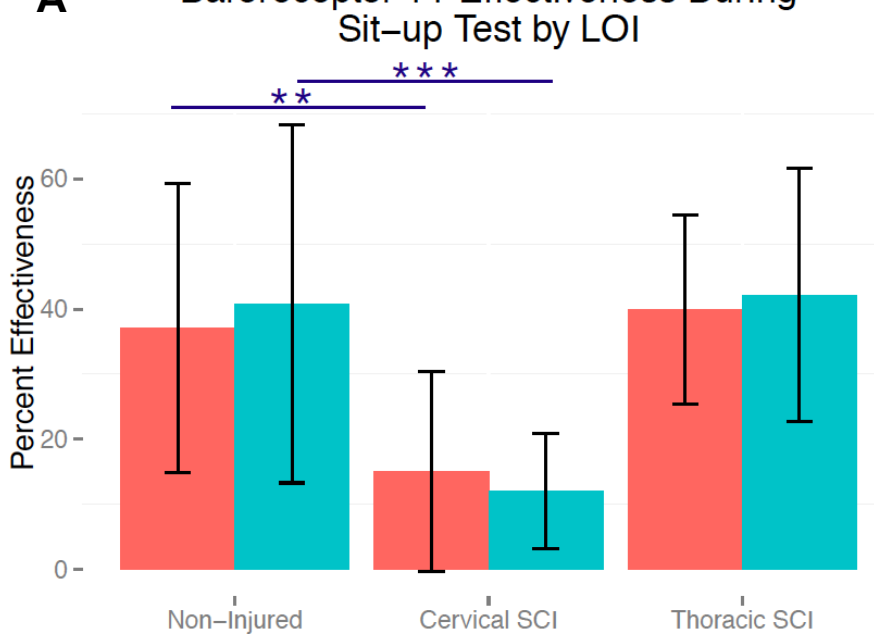

B ++ Baroreceptor Effectiveness

80 - While Sitting by LOI

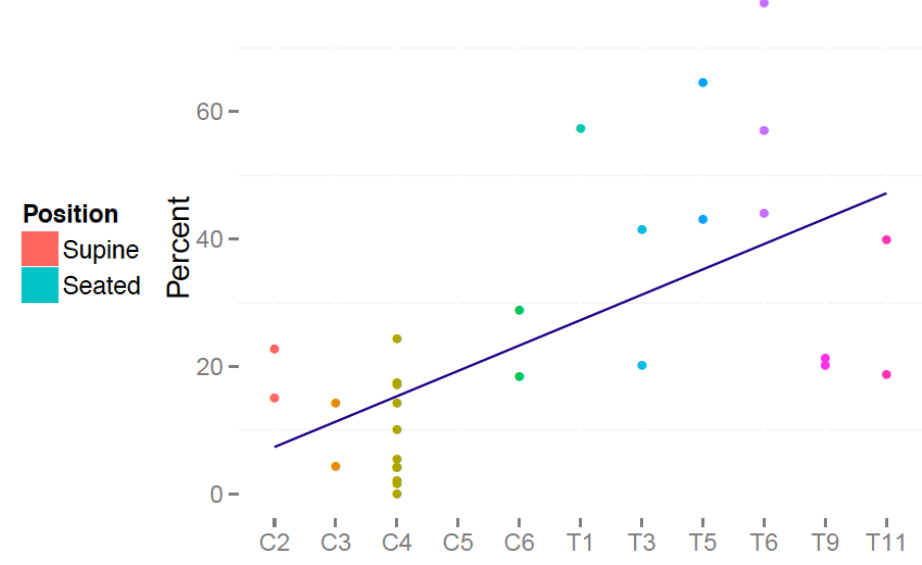

(A) Baroreceptor effectiveness (BEI) was decreased in the cervical group when compared to non-injured (NI), but not in the thoracic group. Effectiveness in the NI group was $37 \pm 22$ percent while supine and $41 \pm 27$ percent while sitting. Cervical BEI was $15 \pm 15$ percent while supine (model 2-A, $p=.002$ ), which decreased to $12 \pm 8$ percent while seated (model 2-A, $p<.001)$. The thoracic group was insignificantly different from $\mathrm{NI}$ while supine $(40 \pm 14$ percent; model 2-A, $p$

$=.77)$ and seated $(42 \pm 19$ percent; model $2-A, p=.89)$. (B) Despite the trend, there was no linear relationship between $\mathrm{BEI}$ and level of injury (model 7-A: $\mathrm{p}=.25, \mathrm{R}^{2}=.58$ ). The $\mathrm{BEl}$ is derived from the number of significant ramp sequences 
(Figure VI-7; ramp sequences with a significant relationship between systolic blood pressure and RR intervall) divided by the number of total ramp sequences. It indicates the effectiveness of cardiovascular regulation, measuring the percentage of systolic ramp increases that are followed by a decrease in heart rate or vasoconstriction. The cervical group was significantly worse than NI, indicating an impairment of the vagal arm of cardiovascular regulation despite the injuries sparing the vagus nerve. $\mathrm{NI} \mathrm{n}=11 ; \mathrm{SCI} \mathrm{n}=47$; values are represented as mean \pm standard deviation. 

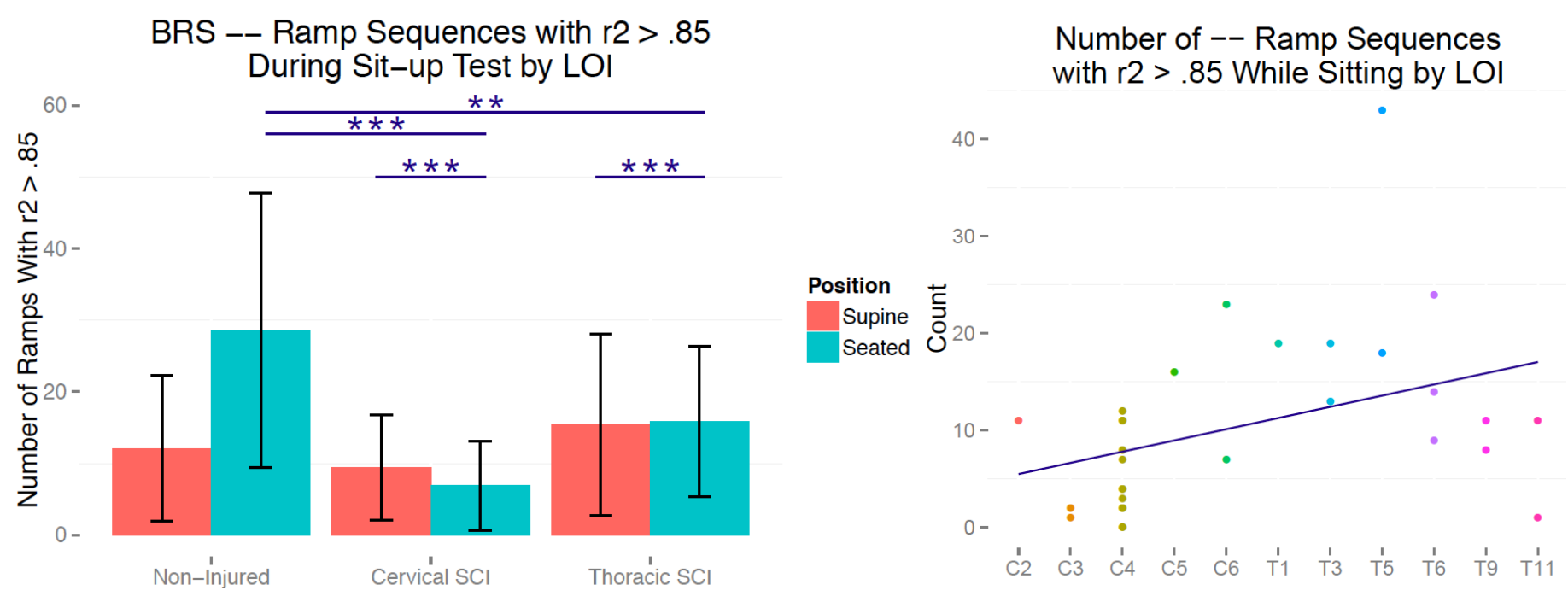

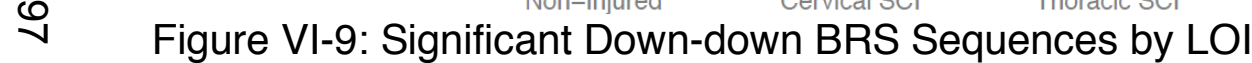

(A) There were significant decreases in the number of significant down-down (down-down*) ramp sequences while seated when comparing the cervical and thoracic groups to non-injured (NI). The NI group had $12 \pm 10$ down-down* ramp sequences while supine, and $29 \pm 19$ down-down* ramp sequences while seated (an increase of $15 \pm 15$ sequences).

The cervical group had $9 \pm 7$ down-down* ramp sequences while supine (model $2-\mathrm{A}, \mathrm{p}=.49$ ), which decreased by $3 \pm 8$ sequences (model $2-A, p<.001$ ) in the seated position to $7 \pm 6$ down-down ${ }^{*}$ sequences (model $2-A, p<.001$ ). The thoracic group had $15 \pm 13$ down-down* ramp sequences while supine (model $2-A, p=.53$ ). While seated, the thoracic 
group had $16 \pm 11$ down-down* ramp sequences (model $2-\mathrm{A}, \mathrm{p}=.01$ ), an increase of $0 \pm 13$ sequences (model 2- $\mathrm{A}, \mathrm{p}=$ .009). (B) There was no significant relationship between the number of down-down* ramp sequences while sitting and level of injury (model 7-A: $p=.66, R^{2}=.50$ ) despite the trend. Down-down* ramp sequences are drops in SBP that are followed by increases in HR and vasoconstriction and are a measurement of the sympathetic arm of cardiovascular regulation. Significant sequences, i.e. sequences with a significant relationship between SBP and $R R I\left(R^{2}>.85\right)$, should increase while seated due to orthostatic stress. While sitting, the cervical group had fewer down-down* ramps, and the thoracic group had the same number of ramps as supine, indicating a decrease in sympathetic nervous system activity during stress when compared to NI. NI $n=11 ; \mathrm{SCI} n=47$; values are represented as mean \pm standard deviation. 

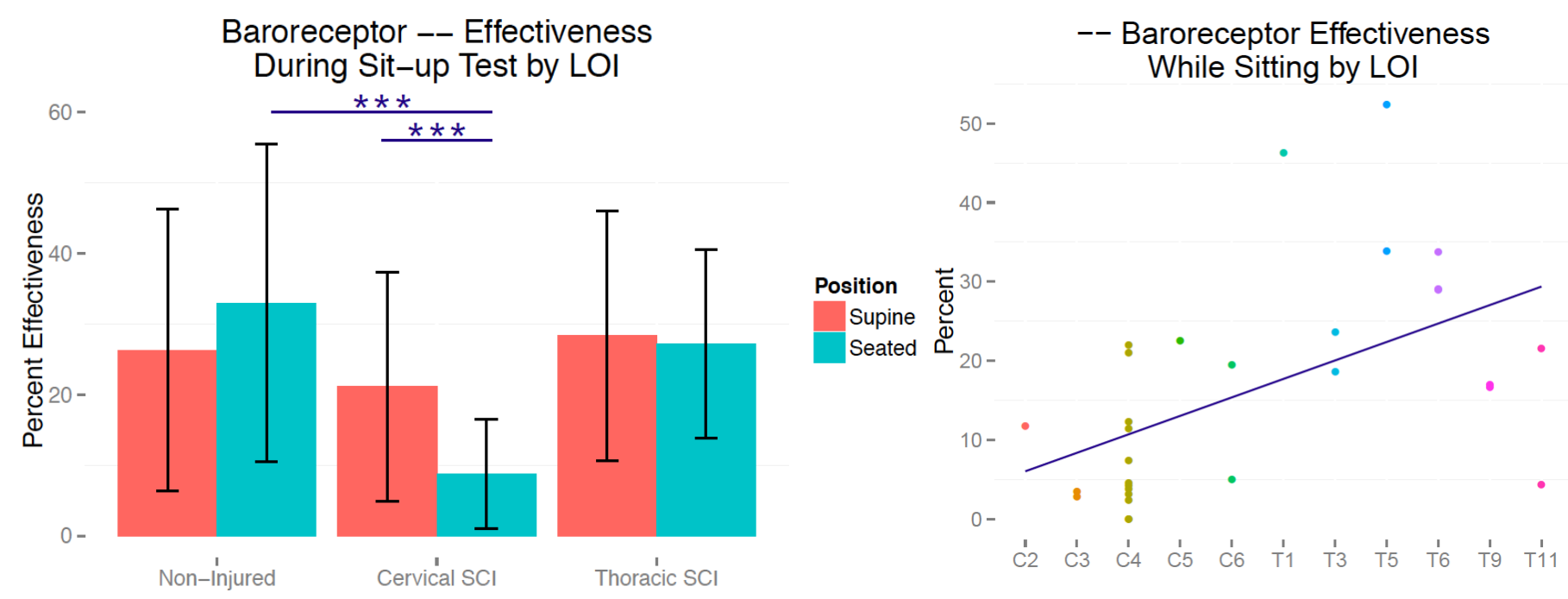

Figure VI-10: Down-down BEI by LOI

(A) There was a significant decrease in the down-down baroreceptor effectiveness index (BEI) when comparing the cervical group to non-injured (NI). Supine, NI BEI was $26 \pm 20$ percent and increased to $33 \pm 22$ percent while sitting (an increase of $7 \pm 19$ percent). The cervical group BEI was similar while supine $(21 \pm 16$ percent; model $2-A, p=.46)$ but dropped by $14 \pm 16$ percent (model $2-A, p<.001$ ) while seated to $9 \pm 8$ percent (model $2-A, p<.001$ ). The supine BEl of the thoracic group was $28 \pm 17$ percent (model $2-\mathrm{A}, \mathrm{p}=.79$ ) and dropped slightly $(1 \pm 17$ percent; model $2-\mathrm{A}, \mathrm{p}=.30)$ to 
$27 \pm 13$ percent while seated (model $2-A, p=.35$ ). (B) Despite the trend, there was no significant relationship between $\mathrm{BEI}$ and level of injury (model $3-\mathrm{A}: \mathrm{p}=.34, \mathrm{R}^{2}=.54$ ). The $\mathrm{BEl}$ is calculated by dividing the number of significant ramp sequences (sequences where the relationship between systolic blood pressure and RR interval is $>.85$ and thus significant) into the total number of systolic blood pressure ramps. The percent that results is therefore a measurement of how many decreases in systolic blood pressure resulted in an increase in heart rate or vasoconstriction. A decrease in percent effectiveness while seated demonstrates that there is an impairment in the sympathetic arm of cardiovascular regulation during stress, particularly in the cervical group. $\mathrm{NI} n=11$; $\mathrm{SCl} n=47$; values are represented as mean \pm standard deviation. 

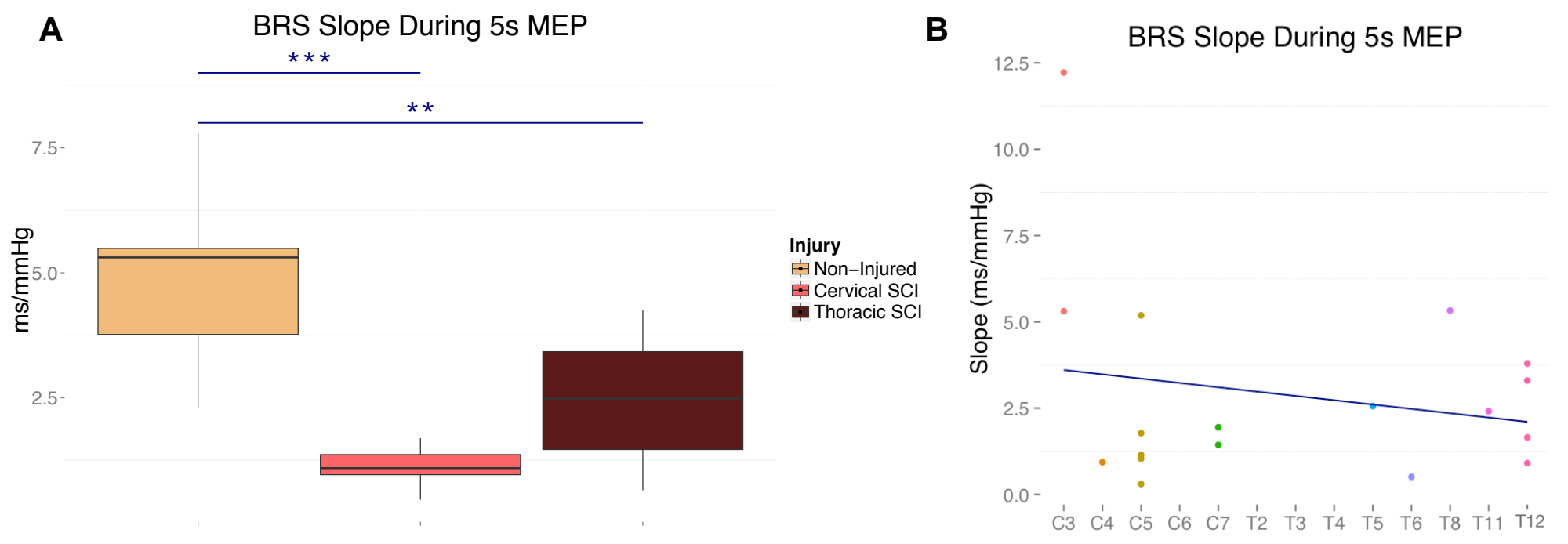

을 Figure VI-11: BRS Slope During 5s MEP by LOI

(A) Baroreceptor Sensitivity (BRS) slope during the 5s MEP was significantly decreased in both the cervical (model 6-A, $p$ $<.001$ ) and thoracic (model 6-A, $\mathrm{p}=.002$ ) groups when compared to the non-injured (NI) BRS slope. The NI slope was 5 $\pm 1 \mathrm{~ms} / \mathrm{mmHg}$, while cervical slope was $1 \pm .5 \mathrm{~ms} / \mathrm{mmHg}$ and thoracic was $3 \pm 2 \mathrm{~ms} / \mathrm{mmHg}$. (B) There was no significant linear relationship between BRS slope and level of injury despite the trend (model 7-A: $p=.72, R^{2}=.09$ ). The slope during the 5s MEP, also called phase 2, demonstrates the ability of the sympathetic nervous system to overcome sustained drops in systolic blood pressure by increasing heart rate. The cervical group, the group with the most overall 
sympathetic dysfunction, has significantly smaller BRS than the thoracic group, which has varying degrees of sympathetic cardiac innervation. $\mathrm{NI} n=11 ; \mathrm{SCI} n=47$; values are represented as mean \pm standard deviation. 

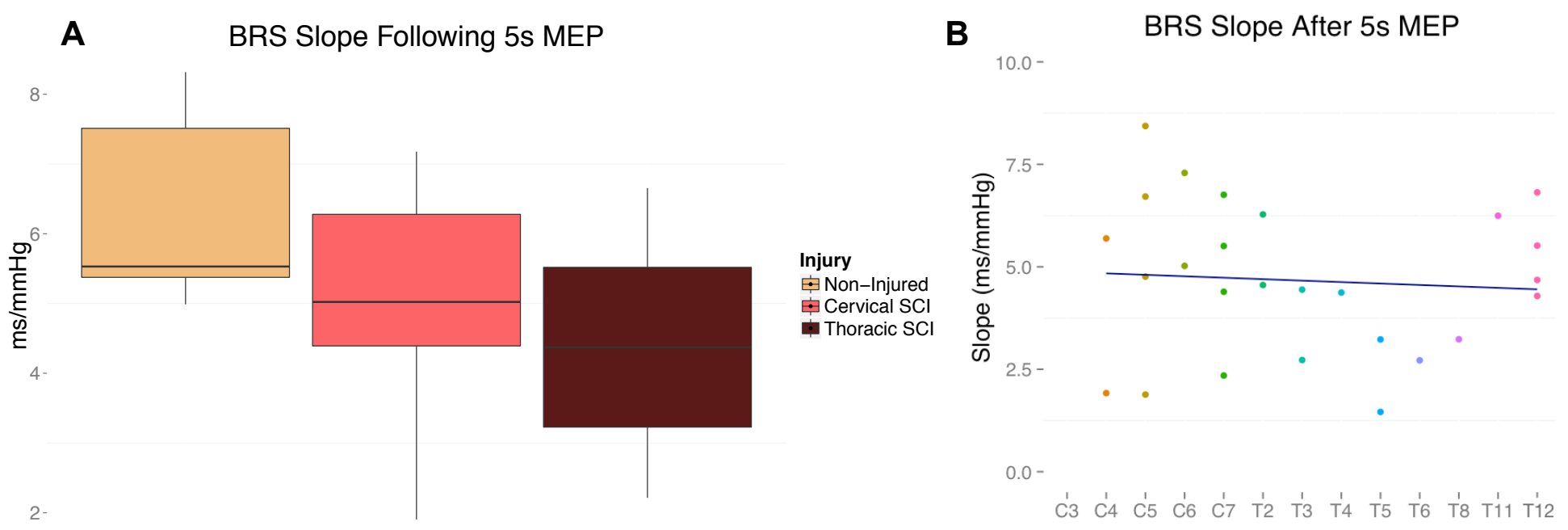

Figure VI-12: BRS Slope Following 5s MEP by LOI

(A) Baroreceptor Sensitivity (BRS) slope following the 5s MEP (phase IV ) was not significantly different than non-injured

(NI) (model $6-\mathrm{A}, \mathrm{p}=.43$ and $\mathrm{p}=.19$ ). The $\mathrm{NI}$ mean slope was $6 \pm 2 \mathrm{~ms} / \mathrm{mmHg}$, while cervical was $5 \pm 2 \mathrm{~ms} / \mathrm{mmHg}$ and thoracic was $4 \pm 2 \mathrm{~ms} / \mathrm{mmHg}$. (B) There was no linear relationship between BRS slope and level of injury (model 7-A: $p=$ $.41, R^{2}=.30$ ). Phase IV illustrates the ability of the parasympathetic nervous system to decrease HR in response to a rapid increase in $\mathrm{SBP} . \mathrm{NI} n=11 ; \mathrm{SCI} n=47$; values are represented as mean \pm standard deviation. 


\section{DISCUSSION}

First, we found that pulmonary function outcomes correlated significantly with level of injury. Others have previously found that pulmonary dysfunction in $\mathrm{SCl}$ is largely due to paralysis of inspiratory and expiratory muscles of respiration (Schilero et al 2009, Tollefsen \& Fondenes 2012), and our results support this: there was a significant linear correlation between level of injury and spirometric outcomes independent of AIS score. Thus, as the lesion descends, more respiratory muscles are available to recruit and pulmonary function increases. All of our participants were free from obstructive respiratory diseases, which could be why pulmonary function was statistically independent of AIS category.

Analysis of HRV demonstrated a significant decrease in sympathetic control over the SA node and decreased vagal activity in the cervical and thoracic group compared to NI participants with no strong linear correlation to level of injury. While supine, there were no significant differences in the LF or HF power between groups, though the powers in the cervical group were lower than both thoracic and NI. Supine, the LF band indicates a balance of SNS and PNS tone over the SA node. Some have found the LF band to be abolished altogether in the cervical group (Inoue et al 1995), but others, like us, found insignificant decreases in LF power when comparing cervical SCI to $\mathrm{NI}(1979$, Bluvshtein et al 2011, Houtman et al 2000, Wecht et al 2006). Since the LF band includes both SNS and PNS activity, it's hard to determine which is contributing to the decrease as persons with cervical and thoracic SCl exhibit impaired SNS activity as well as 
varying degrees of vagal withdrawal. Likewise, there were no significant differences between groups when comparing supine, HF RRI power, although the thoracic and cervical values were both lower than the NI values. The HF band is the result of PNS activity, which could explain why there was no difference while supine.

Most of the deficits to autonomic balance were demonstrated while upright. While passively upright, $\mathrm{HR}$ increases due to a simultaneous increase in SNS activity and decrease in PNS activity at the SA node. Unlike supine values, the LF band while upright is entirely indicative of SNS control. Our NI group had no increase in SNS activity while upright, but they demonstrated a large decrease in vagal activity. The cervical group demonstrated a significant drop in SNS activity while upright, indicative of impairment to descending sympathetic cardiac neurons. There was also an overall decrease in SNS activity demonstrated in the thoracic group, which is likely because most of the injuries were at or above the level where the sympathetic cardiac neurons emerge.

There were no significant differences between groups with regard to HF power, indicating that all groups attempted to increase HR by vagal inhibition; although the decrease in the cervical group was the greatest, it was not significant. This could be compensatory due to inability of the SNS to increase HR. And unlike previous researchers, upon assumption of an upright posture our cervical group demonstrated a significant decrease in RRI when compared to the $\mathrm{NI}$ group. Others found that cervical groups are incapable of decreasing RRI 
whilst upright despite vagal withdrawal. This indicates that vagal desensitization of the SA node seen by other researchers might not be evident in our cervical group.

BRS sequence analysis demonstrated that there were significant impairments to cardiovascular regulation in the cervical and thoracic groups without a linear relationship to level of injury. Supine, the significant up-up (up$u^{*}$ ) and significant down-down (down-down*) ramps are almost entirely due to the cardiovagal loop of the baroreceptor reflex, either by inhibition of the vagus (down-down*) in the NTS or by removal of vagal inhibition (up-up*) (Bertinieri et al 1985, Parati et al 2000). Both cervical and thoracic groups had significantly fewer up-up* and down-down* ramps while seated, and the change in ramps from supine to sitting was significantly different in both groups when compared to NI. While sitting, down-down* ramps result from SNS engagement (largely baroreceptors and cardiopulmonary receptors), while up-up* ramps result from vagal activity (Blomqvist 2011, O'Leary et al 2003). Up-up and down-down BEls were also significantly decreased in the cervical group, supine and seated, compared to NI, meaning the cervical group had a great number of SBP oscillations that did not elicit a response from the baroreceptors. Both cervical and thoracic SCl participants, therefore, experienced an impaired sympathetic and parasympathetic engagement due to poor respiratory motor control.

Finally, we found that analysis of the 5s MEP maneuver demonstrated impairments to baroreceptor activation which correlated to neither level nor 
completeness of injury. Cervical and thoracic participants demonstrated a significantly decreased BRS slope during the 5s MEP maneuver when compared to NI. There were no significant differences between cervical and thoracic groups when comparing $\mathrm{BRS}$ following the $5 \mathrm{~s} \mathrm{MEP}$ to $\mathrm{NI}$, despite there being significant differences to $\mathrm{SCl}$ overall. These deficits are the result of baroreceptor dysfunction, as the increased intrathoracic pressure during expiration inhibits activity of the cardiopulmonary and low-pressure receptors. Thus, decreased BRS could be secondary to $\mathrm{SCl}$ and not entirely dependent on level of injury or AIS impairment.

These decreases demonstrate, following SCI, reflexes responsible for maintaining blood pressure are engaged significantly less than they are in $\mathrm{NI}$ persons, both at rest and during stress. The decreased engagement correlates with neither level of injury nor AIS impairment, further confirming dysfunction is exacerbated by poor pulmonary function and not dependent on the level or completeness of injury.

This additional dysfunction in SCl has the added benefit, however, of plasticity: in NI persons, improvements to cardiovascular function occur after targeting respiratory motor control (Tamplin \& Berlowitz 2014, Van Houtte et al 2006). The dependence of cardiovascular dysfunction on pulmonary dysfunction means in SCI this relationship still exists and thus has the potential to be reversed. 


\title{
CHAPTER VII:
}

\section{CARDIOVASCULAR REGULATION POST RESPIRATORY MOTOR TRAINING}

\section{INTRODUCTION}

\begin{abstract}
After our discovery that pulmonary function does indeed impair cardiovascular function, particularly at the level of the baroreceptors, we attempted to discover the plasticity inherent therein: our first aim was to discover any changes in cardiovascular function that occur secondary to an improvement in pulmonary function, while the second aim was to use any changes that occur to better understand the mechanism of cardiovascular dysfunction from poor respiratory motor control. Our hypothesis was that an improvement to pulmonary function can improve cardiovascular regulation due to their interdependence.
\end{abstract}

\section{METHODS AND PROTOCOL}

Participants in the Respiratory Motor Training (RMT; Table III-2, 39) group and the control group (Table III-3, 40) were assessed of pulmonary and cardiovascular outcomes during screening a Respiratory Motor Control 
Assessment (RMCA, 42) and Orthostatic Stress Test (Orthostatic Stress Test, 47). Participants that were in the RMT group then participated in twenty sessions of inspiratory and expiratory training, using both Positive Expiratory Pressure and Inspiratory Muscle Training Devices (PEP and IMT, Respironics, Murraysville, PA) with the goal of the participant breathing at $60 \%$ of his maximum airway pressure (MEP) by the last session. After twenty sessions of RMT, participants were then assessed of follow-up pulmonary function and cardiovascular regulation outcomes. Participants in the control group were assessed four to six weeks post-screening assessment. Control participants maintained their own fitness and therapy regimens, provided it did not include electrical stimulation or locomotor-training therapies.

Screening and follow-up assessments, called Pre-test and Post-test, respectively, measured spirometric outcomes and airway pressure generation; hemodynamic and heart rate responses to the orthostatic stress test; heart rate variability (HRV) and autonomic tone; and baroreceptor sensitivity (BRS), as measured by the sustained, maximum expiratory pressure (5s MEP) maneuver and baroreceptor sequencing method. The change from pre- to post-test was used to compare the RMT group to control in multivariate linear regression analyses (Table III-5); values are reported as mean \pm standard deviation.

\section{RESULTS}

There were significant improvements to pulmonary function outcomes in the RMT group post-test that were not seen in controls (Figure VII-1). 
Additionally, there were significant improvements in hemodynamics during the orthostatic stress test independent of heart rate (Figure VII-4, Figure VII-5, Figure VII-6): the RMT group ameliorated their drop in systolic blood pressure (SBP) while upright, and significantly increased their diastolic blood pressure (DBP) values, without increases in heart rate.

There were no changes in sympathetic tone post-RMT (Figure VII-2), however, there was a significant increase in parasympathetic tone from pre- to post-test while upright in the RMT group (Figure VII-3). The BRS sequencing method found significant increases in the number of seated, significant up-up (up-up*) and down-down (down-down*) ramp sequences (Figure VII-8, Figure VII-11) which led to a significantly increased down-down baroreceptor effectiveness index (BEl; Figure VII-12); there were no significant changes from pre- to post-test in up-up BEI (Figure VII-9). In addition to the increased number of significant ramps, there were increases in the slopes (in $\mathrm{ms} / \mathrm{mmHg}$ ) of these ramps, despite the pre-test values being no different than non-injured values (Figure VII-7, Figure VII-10).

Finally, there were significant increases in BRS outcomes obtained during and after the 5s MEP (Figure VII-13, Figure VII-14) that were not seen in the control group. 


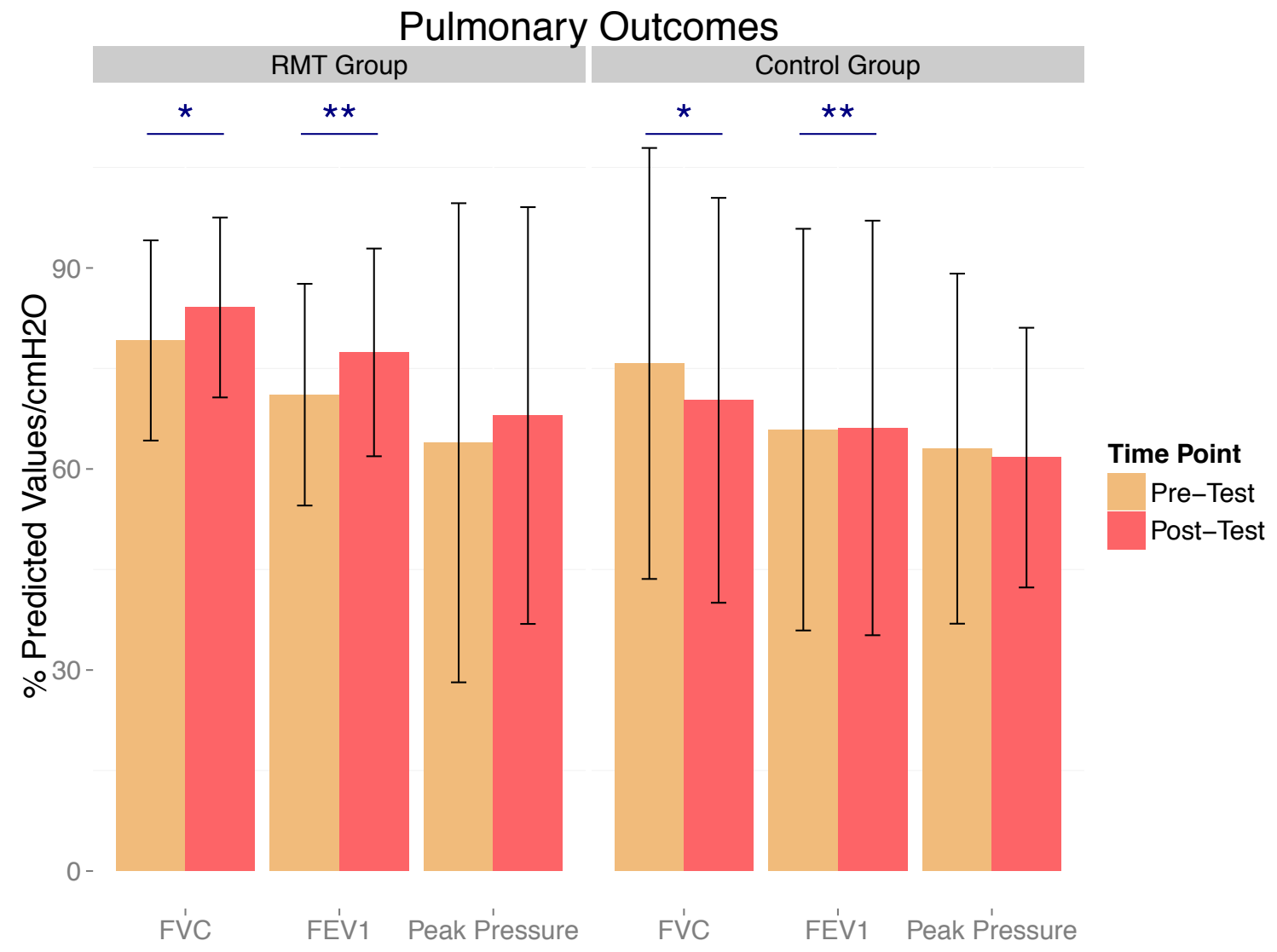

Figure VII-1: Pulmonary Function Outcomes Pre- and Post-RMT

There were significant increases in pulmonary function outcomes in the respiratory motor training (RMT) group when compared to control. The percentpredicted forced vital capacity (FVC) values pre-test were $79 \pm 15$ in the RMT group, and increased to $84 \pm 13$ percent in the post-test (model 2-B, $\mathrm{p}=.02$ ). The control group FVC percent decreased from $76 \pm 32$ pre-test to $70 \pm 30$ post-test. The percent-predicted forced expiratory volume in $1 \mathrm{~s}\left(\mathrm{FEV}_{1}\right)$ values also increased in the RMT group from $71 \pm 17$ percent pre-test to $77 \pm 16$ percent post-test (model $2-B, p=.008$ ) while the control group remained nearly the same from $66 \pm 30$ pre-test to $66 \pm 31$ post-test. Finally, peak airway pressure 
generation (MEP, in $\mathrm{cmH}_{2} \mathrm{O}$ ) demonstrated no significant increase, from $64 \pm 36$ $\mathrm{cmH}_{2} \mathrm{O}$ pre-test to $68 \pm 31 \mathrm{cmH}_{2} \mathrm{O}$ post-test (model 6-B, $\mathrm{p}=.09$ ). Control group decreased peak airway pressure generation from pre- to post-test $(63 \pm 26$ $\mathrm{cmH}_{2} \mathrm{O}$ to $62 \pm 19 \mathrm{cmH}_{2} \mathrm{O}$, respectively). In addition to illustrating pulmonary function, pulmonary function outcomes indicate the amount of respiratory motor control in SCl. The significant increases from pre- to post-test in the RMT group, indicate that RMT not only improves pulmonary function in $\mathrm{SCl}$ but it also improves respiratory motor control. RMT $n=25$; control $n=22$; values are represented as mean \pm standard deviation. 


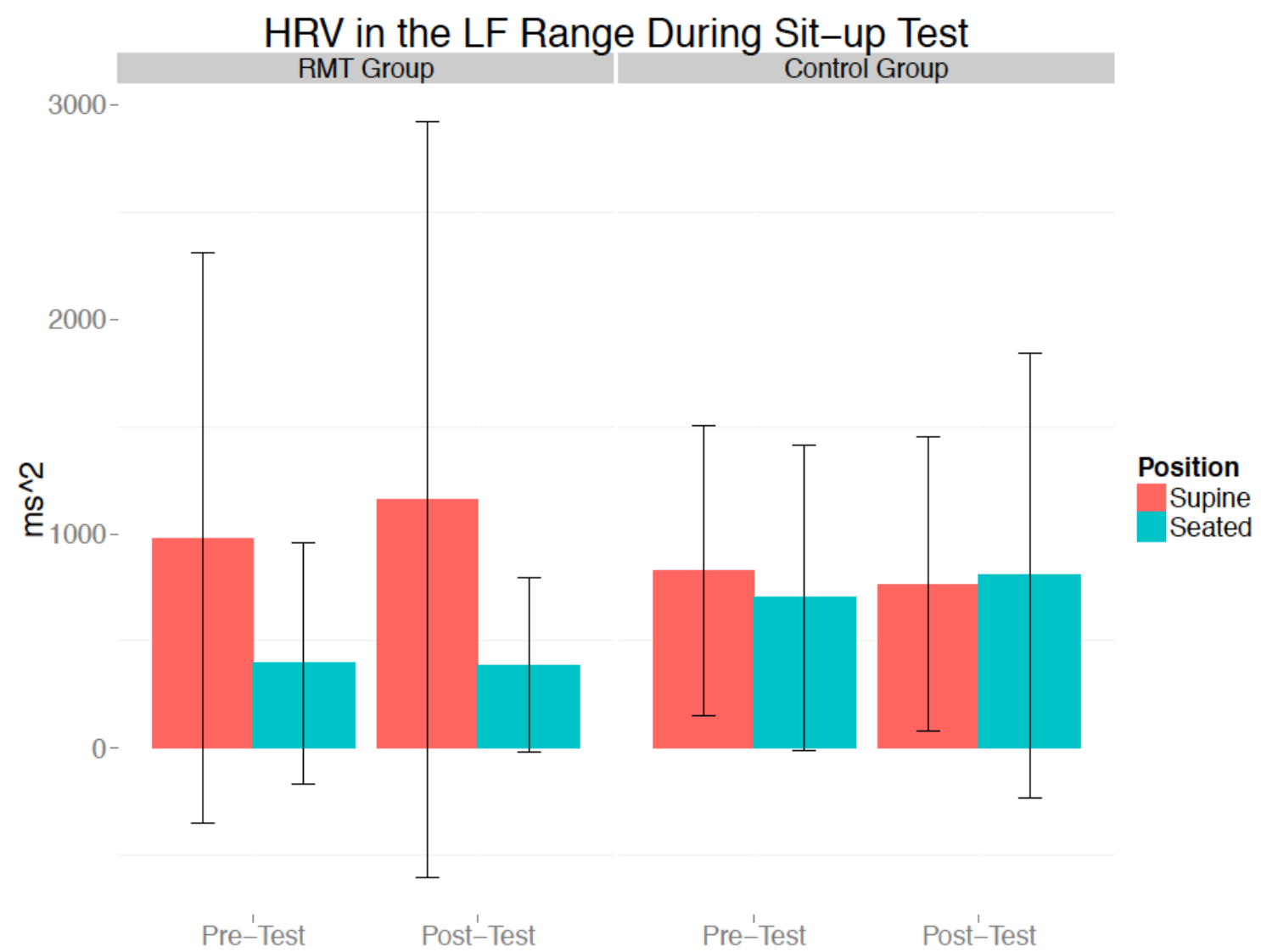

Figure VII-2: Sympathetic HRV Pre- and Post-RMT

There were no significant changes in low-frequency heart rate variability (LF HRV) when comparing the respiratory motor training (RMT) group to the control. Pre-test supine, LF HRV in the RMT group was $980 \pm 1331 \mathrm{~ms}^{2}$, while control was $827 \pm 181 \mathrm{~ms}^{2}$. LF HRV dropped while seated in the RMT group to $368 \pm$ $759 \mathrm{~ms}^{2}$ and in the control group to $702 \pm 714 \mathrm{~ms}^{2}$. Post-test supine values in the RMT group were $1199 \pm 1804 \mathrm{~ms}^{2}$ and $766 \pm 686 \mathrm{~ms}^{2}$ in control (model 1-B, $\mathrm{p}=$ .79). LF HRV dropped while seated in the RMT group to $279 \pm 321 \mathrm{~ms}^{2}$ and increased while seated in the control to $807 \pm 1037 \mathrm{~ms}^{2}$ (model 1-B, $p=.94$ ). LF $\mathrm{HRV}$ is a measurement of control exerted by the sympathetic nervous system 
over RRI. There was no post-test increase in sitting LF power in the RMT group when compared to the control; therefore it is unlikely that RMT caused any changes in sympathetic nervous system activity. RMT $n=25$; control $n=22$; values are represented as mean \pm standard deviation 


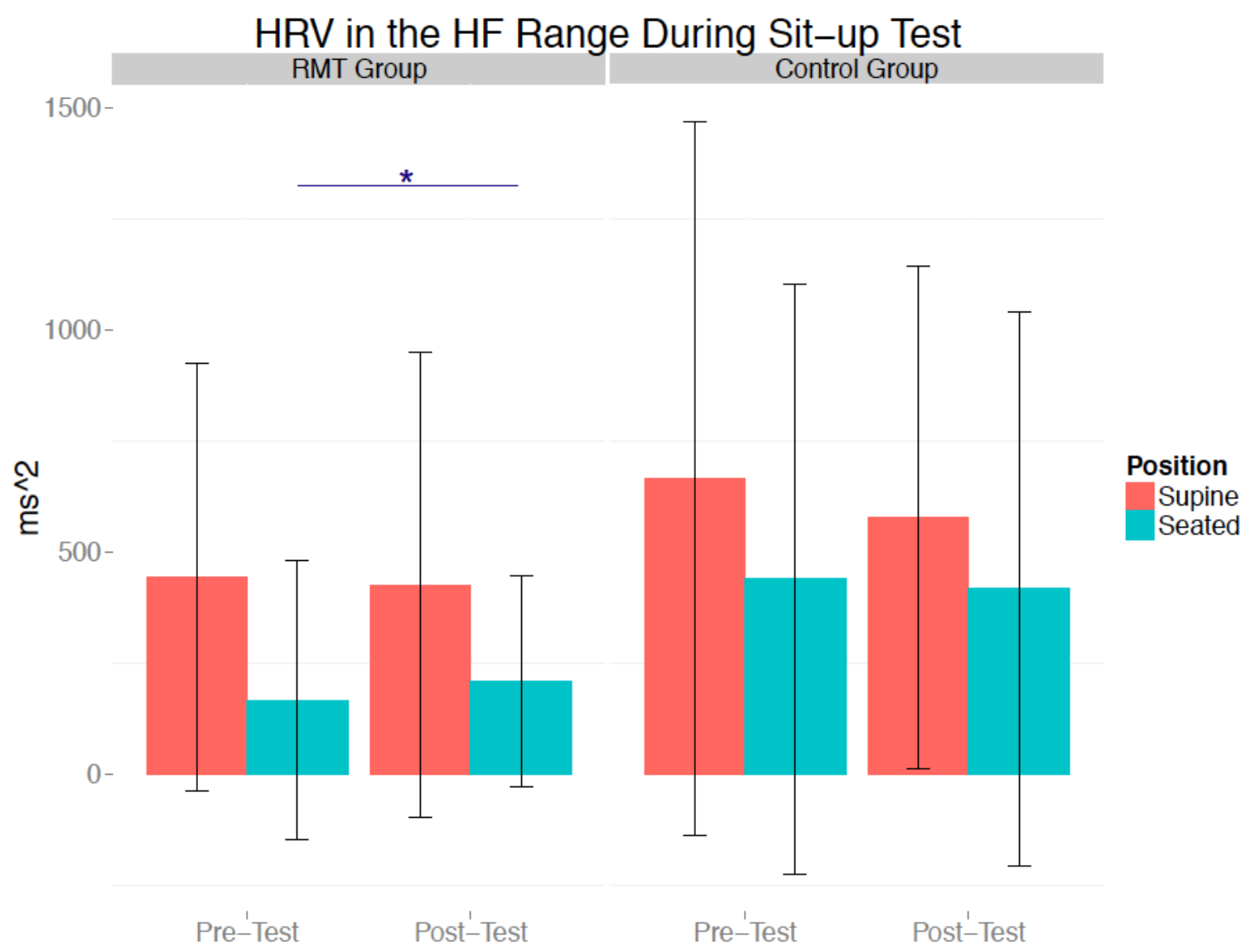

Figure VII-3: Parasympathetic HRV Pre- and Post-RMT

There were no significant changes in high-frequency heart rate variability (HF HRV) while supine when comparing the respiratory motor training (RMT) group to control; however, there was a significant increase in sitting HF HRV from pre-test to post-test when comparing the RMT and control groups. Supine, pre-test HF HRV was $442 \pm 480 \mathrm{~ms}^{2}$ in the RMT group. That decreased slightly to $424 \pm 523$ $\mathrm{ms}^{2}$ in the post-test (model 6-B, $\mathrm{p}=.27$ ). In the control group, pre-test HF HRV was $663 \pm 803 \mathrm{~ms}^{2}$ and post-test HF HRV was $576 \pm 564 \mathrm{~ms}^{2}$. Sitting, however, increased significantly (model $6-\mathrm{B}, \mathrm{p}=.03$ ) from $166 \pm 314 \mathrm{~ms}^{2}$ to $208 \pm 237 \mathrm{~ms}^{2}$ in the RMT group while the control remained nearly unchanged at $438 \pm 663 \mathrm{~ms}^{2}$ pre-test to $417 \pm 623 \mathrm{~ms}^{2}$ post-test. HF HRV is a measurement of 
parasympathetic control over heart rate, particularly the generation of the respiratory sinus arrhythmia. An increase while sitting is most likely an increase in activity of the cardiopulmonary receptors which control RRI through vagal efferents, as widespread increases in parasympathetic activity would be demonstrated while supine in addition to sitting. RMT $n=25$; control $n=22$; values are represented as mean \pm standard deviation. 


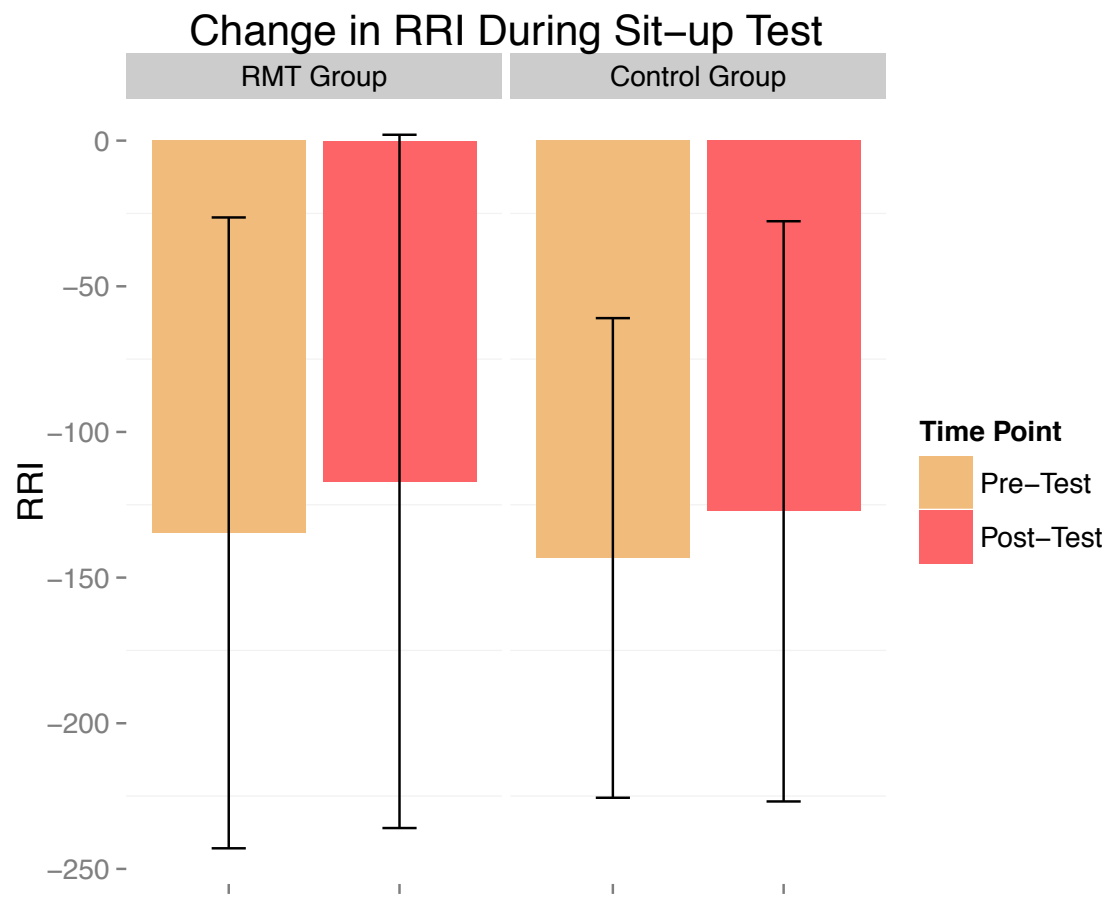

Figure VII-4: Change in RR Interval During Orthostasis Pre- and Post-RMT

Following respiratory motor training (RMT), there were no significant differences in RR interval (RRI) response to orthostasis when compared to the control posttest values. Prior to RMT, RRI decreased by $134 \pm 108$ ms while seated. Post RMT, RRI decreased by $117 \pm 119 \mathrm{~ms}$. The control group pre-test RRI decreased by $143 \pm 82 \mathrm{~ms}$, while post-test decreased $127 \pm 100 \mathrm{~ms}$. RMT $n=25$; control $n$ $=22$; values are represented as mean \pm standard deviation . 


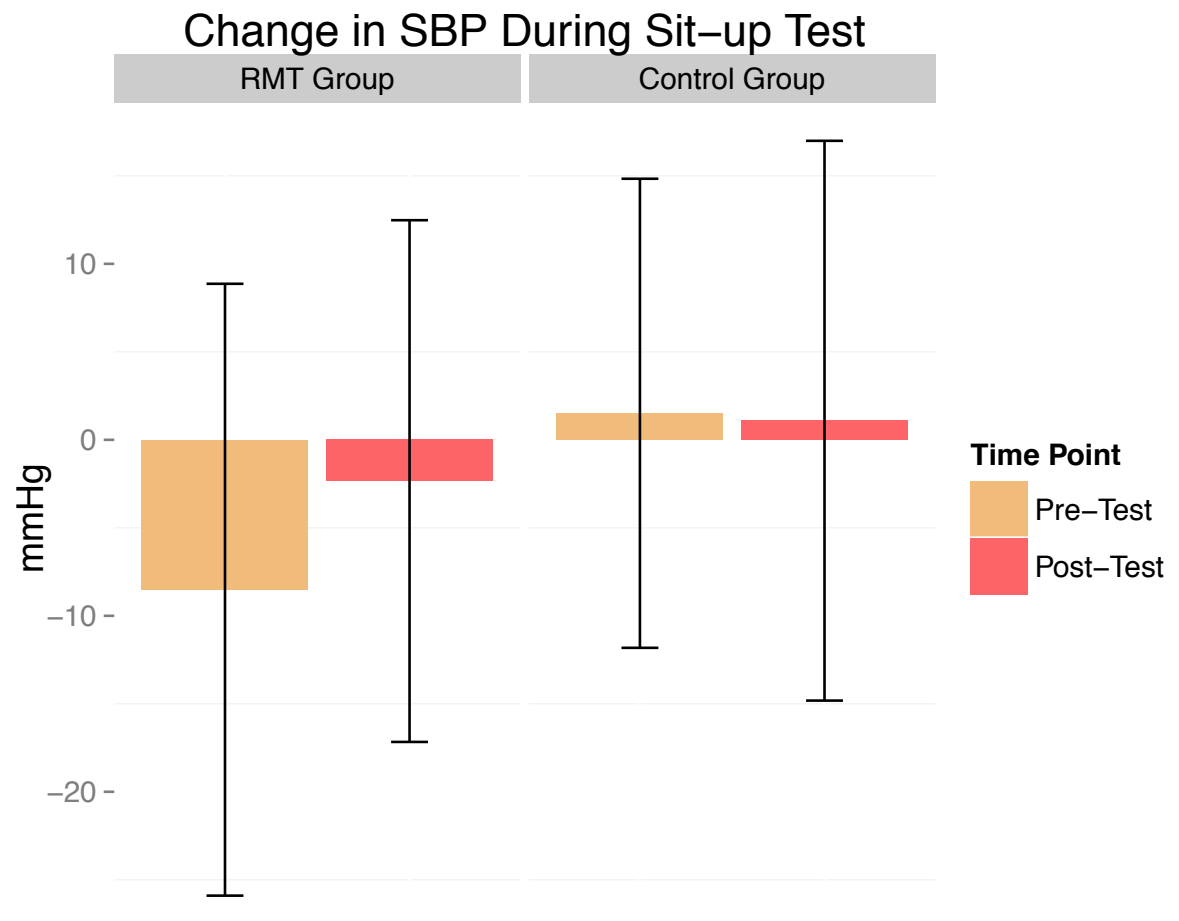

Figure VII-5: Change in SBP During Orthostasis Pre- and Post-RMT

The drop in systolic blood pressure (SBP) experienced while upright in the respiratory motor training (RMT) group was ameliorated following RMT, while the control group experienced no such changes between pre- and post-test values. When the thoracic participants were excluded, however, the change in SBP in the RMT group from pre- to post was significant (model 4-B, $p=.03$ ). In the RMT pre-test, SBP dropped by $9 \pm 17 \mathrm{mmHg}$ while seated; following RMT, SBP dropped by $2 \pm 15 \mathrm{mmHg}$ while upright. There were likewise no changes in SBP values from pre- to post-tests in the control group: pre-test SBP increased by $2 \pm$ $13 \mathrm{mmHg}$ while seated, and post-test SBP increased by $1 \pm 16 \mathrm{mmHg}$. RMT $\mathrm{n}=$ 25 ; control $n=22$; values are represented as mean \pm standard deviation. 


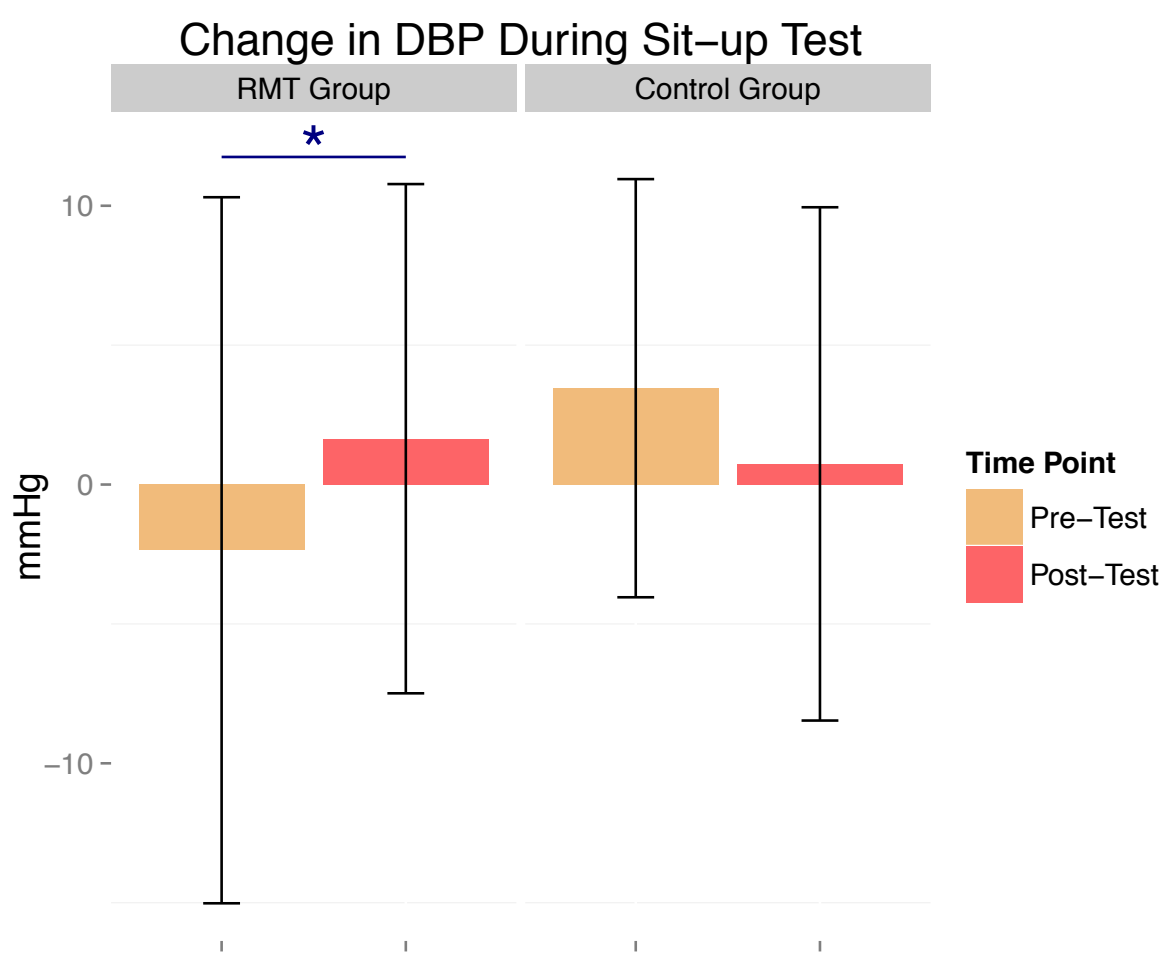

Figure VII-6: Change in DBP During Orthostasis Pre- and Post-RMT The change in DBP while seated improved significantly in the RMT group compared to control. Prior to RMT, DBP dropped by $2 \pm 12 \mathrm{mmHg}$ while seated. Post-RMT, DBP increased by $2 \pm 9 \mathrm{mmHg}$ (model 2-B, $\mathrm{p}=.03$ ). The control group pre-test DBP increased by $3 \pm 8 \mathrm{mmHg}$, while the post-test DBP increased by $1 \pm 9 \mathrm{mmHg}$. The RMT group was therefore able to significantly improve their cardiovascular regulation in response to orthostasis, and maintain their DBP while upright. RMT $n=25$; control $n=22$; values are represented as mean \pm standard deviation. 


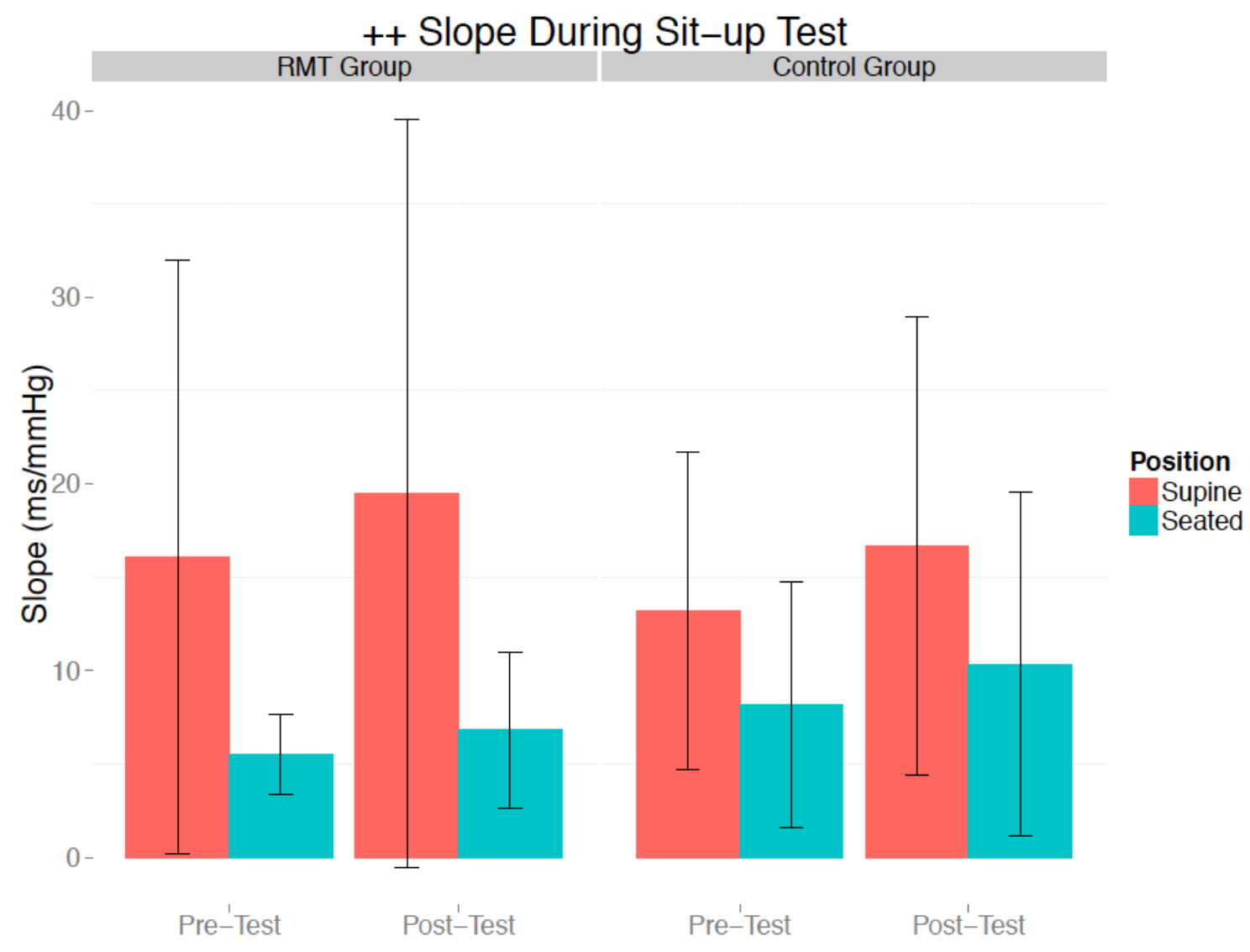

Figure VII-7 Up-up BRS Sequence Slopes Pre- and Post-RMT

There were no significant changes in up-up slope from pre- to post-test when comparing respiratory motor training (RMT) group to control. While supine, up-up slope increased from $16 \pm 16 \mathrm{~ms} / \mathrm{mmHg}$ pre-test to $20 \pm 20 \mathrm{~ms} / \mathrm{mmHg}$ post-test in the RMT group (model $5-B, p=.16$ ), while the control group increased from 13 $\pm 9 \mathrm{~ms} / \mathrm{mmHg}$ pre-test to $17 \pm 12$ post-test. Seated, up-up slope increased from 5 $\pm 2 \mathrm{~ms} / \mathrm{mmHg}$ to $7 \pm 4 \mathrm{~ms} / \mathrm{mmHg}$ (model $5-\mathrm{B}, \mathrm{p}=.31$ ). The control group increased from $8 \pm 7$ to $10 \pm 9$ while seated. The up-up slope illustrates the relationship between increases in RRI in response to increases in SBP coordinated by the baroreceptors (with some influence from the cardiopulmonary 
receptors). RMT $n=25$; control $n=22$; values are represented as mean \pm standard deviation. 


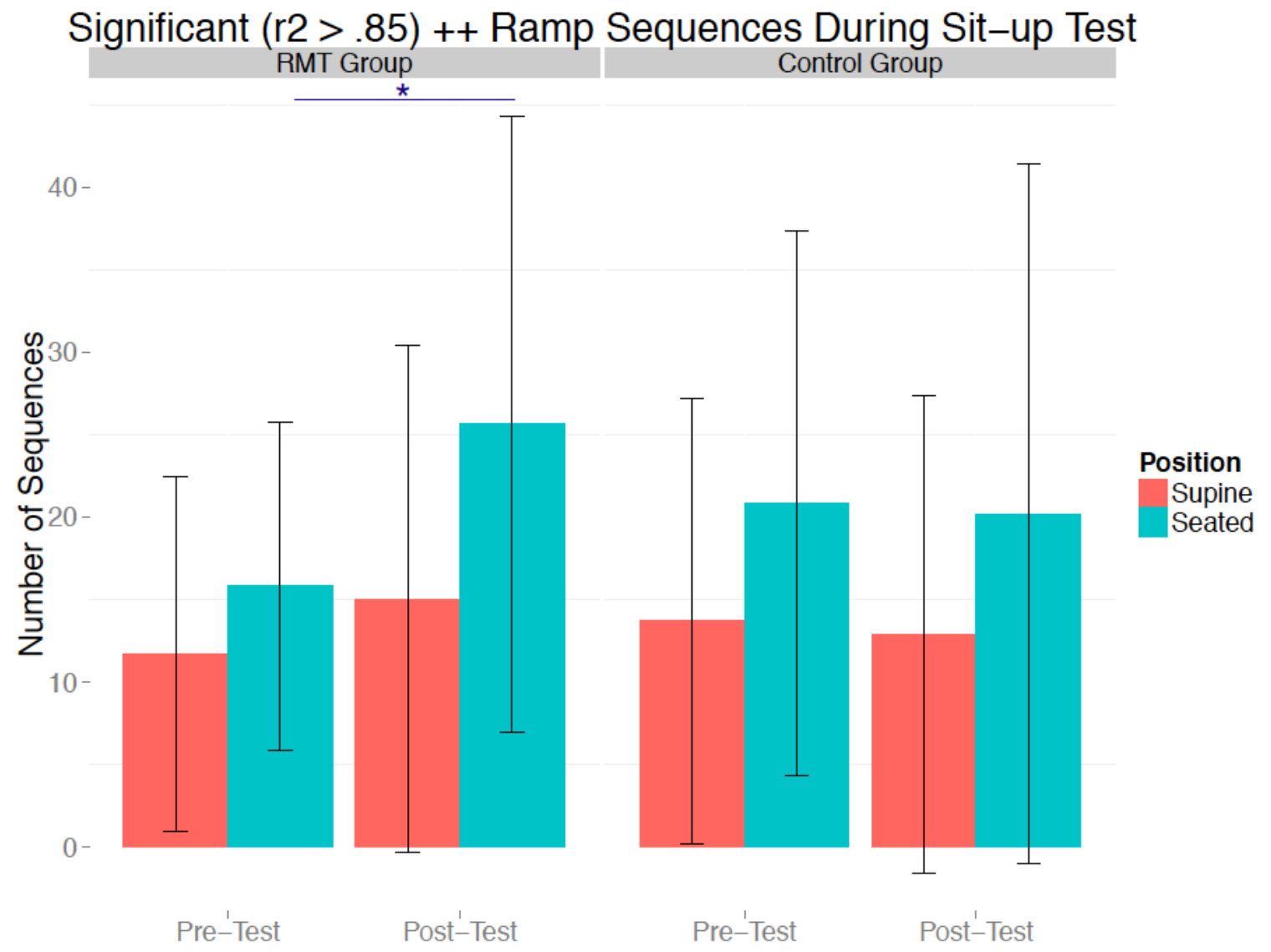

Figure VII-8 Significant Up-up BRS Sequences Pre- and Post-RMT

There was a significant increase in the number of significant up-up (up-up*) ramps while seated when comparing the respiratory motor training (RMT) group to control. Supine, the RMT group had $12 \pm 11$ up-up* ramps in the pre-test, which increased to $15 \pm 15$ up-up* ramps in the post-test (model 2-B, $p=.68$ ). The control group had $14 \pm 13$ up-up* ramps in the pre-test, and $15 \pm 15$ ramps in the post-test. Whilst seated, however, the RMT group increased from $16 \pm 10$ upup* ramps pre-test to $26 \pm 19$ post-test (model $2-B, p=.04$ ). The control group, by contrast, had $21 \pm 16$ up-up* ramps pre-test and $20 \pm 21$ up-up* ramps post- 
test. Up-up* ramps are ramp sequences where the relationship between the increase in BP and increase in RRI is significant $\left(R^{2}>.85\right)$. It is a measurement of vagal control of cardiovascular regulation. The vagus is typically less active during stress, therefore the significant increase in the seated position from pre- to post-test could be either a reversal of vagal withdrawal common to spinal cord injury, or increased activity of the cardiopulmonary receptors due to respiration. RMT $n=25$; control $n=22$; values are represented as mean \pm standard deviation. 


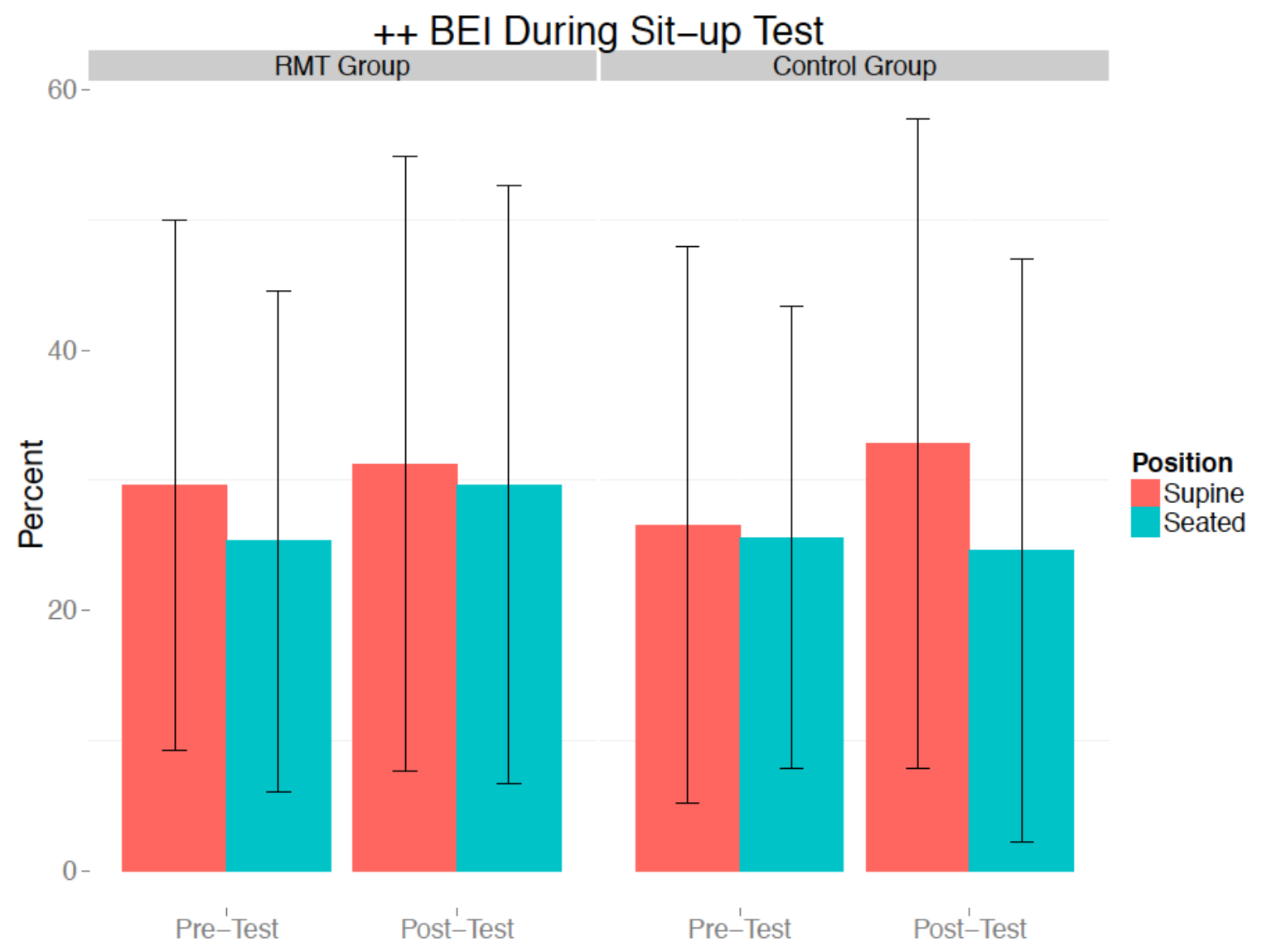

Figure VII-9: Up-up BEI Pre- and Post-RMT

There were increases in baroreceptor effectiveness index (BEI) from pre to post in the respiratory motor training (RMT) group, but they were not significant when compared to control. Supine, BEl in the RMT group was $30 \pm 20$ percent in the pre-test, and $31 \pm 27$ percent in the post-test (model 1-B, $p=.60$ ). The control group was $27 \pm 21$ percent while supine in the pre-test, and $33 \pm 25$ percent in the post-test. In the seated position, the RMT group was $25 \pm 19$ percent pre-test and $30 \pm 23$ percent post-test (model $1-B, p=.18$ ). The control group pre-test was $26 \pm 18$ in the seated position and $25 \pm 22$ percent in the post-test. BEl is a measurement of baroreceptor effectiveness obtained by dividing the number of significant up-up ramp sequences (i.e., ramp sequences where the baroreceptors 
were engaged and effectively increased RR interval in response to an increase in systolic blood pressure) into the total number of sequences. There were no significant differences in up-up BEI, despite a significant increase in the number of significant up-up ramp sequences (Figure VII-8), which could have resulted from a concurrent increase in the number of insignificant ramps from pre- to posttest. RMT $n=25$; control $n=22$; values are represented as mean \pm standard deviation. 


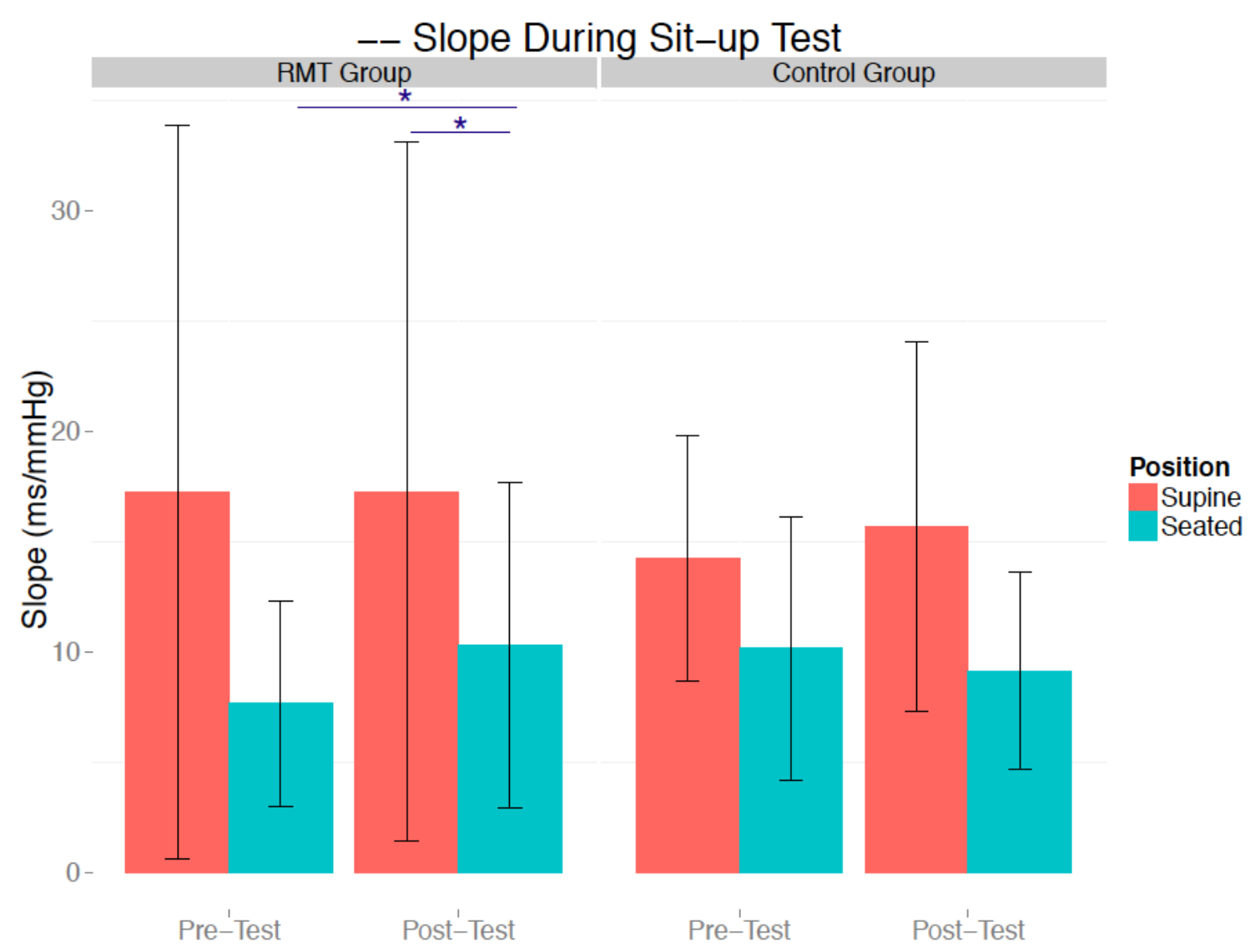

Figure VII-10: Down-down BRS Sequence Slopes Pre- and Post-RMT

There was a significant increase in the slope of down-down baroreceptor sensitivity (BRS) ramp sequences from pre-to post-test in the respiratory motor training (RMT) group when compared to control, as well as a significant decrease in the drop in slope experienced upon sitting. While supine, the pre-test slope for the RMT group was $17 \pm 17 \mathrm{~ms} / \mathrm{mmHg}$ and $17 \pm 16 \mathrm{~ms} / \mathrm{mmHg}$ in the post-test (model 2-B, $p=.26$ ). The control group pre-test slope was $14 \pm 5 \mathrm{~ms} / \mathrm{mmHg}$ and $16 \pm 8 \mathrm{~ms} / \mathrm{mmHg}$ in the post-test. In the seated position, the RMT group pre-test was $8 \pm 5 \mathrm{~ms} / \mathrm{mmHg}$, which increased to $10 \pm 7 \mathrm{~ms} / \mathrm{mmHg}$ in the post-test (model $2-B, p=.02)$. Additionally, the drop in slope from supine to seated decreased significantly in the RMT group. Pre-test, the slope dropped by $-10 \pm 18 \mathrm{~ms} / \mathrm{mmHg}$ 
in the RMT group, but that drop was ameliorated in the post-test to $-7 \pm 17$ $\mathrm{ms} / \mathrm{mmHg}$ (model 2-B, $\mathrm{p}=.05$ ). In the control group, slope dropped by $-5 \pm 5$ $\mathrm{ms} / \mathrm{mmHg}$ in the pre-test, and by $-7 \pm 7 \mathrm{~ms} / \mathrm{mmHg}$ in the post-test. The downdown slope is a measurement of cardiovascular regulation: specifically, the decrease in RR interval (in $\mathrm{ms}$ ) for every $1 \mathrm{mmHg}$ decrease in systolic blood pressure resulting from engagement of baroreceptors and other reflex responses. An increase in the seated position suggests an improvement in the ability of the sympathetic nervous system to increase heart rate and vasoconstriction in response to stress following RMT. RMT $n=25$; control $n=22$; values are represented as mean \pm standard deviation. 


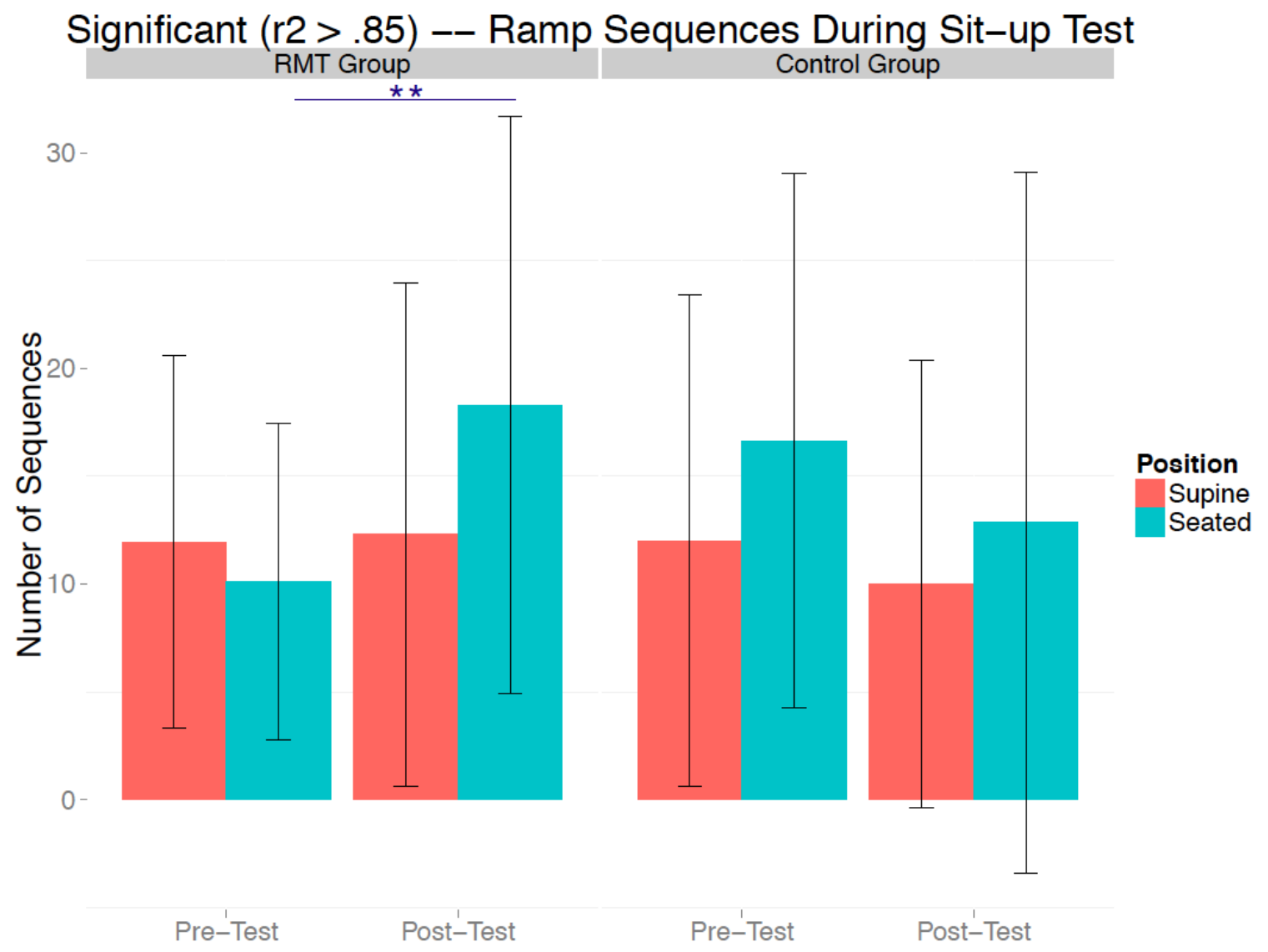

Figure VII-11: Significant Down-down BRS Sequences Pre- and Post-RMT

There was a significant increase in the number of significant down-down baroreceptor sensitivity (BRS) ramp sequences in the respiratory motor training (RMT) group when compared to control. Supine, there were $12 \pm 9$ significant ramps in the RMT group pre-test, and $12 \pm 12$ significant ramps in the post-test (model 4-B, $\mathrm{p}=.48$ ). In the control group, significant ramps while supine decreased from $12 \pm 11$ in the pre-test to $10 \pm 10$ significant ramps in the posttest. While sitting, the number of ramps increased significantly in the RMT group, from $10 \pm 7$ pre-test to $18 \pm 13$ post-test (model $4-B, p=.01$ ). In the control group, the number of significant BRS ramps decreased from $17 \pm 12$ pre-test to $13 \pm 16$ post-test. Significant down-down ramp sequences are the number of 
decreasing systolic blood pressure ramps that resulted in a decrease in $R R$ interval, provided that the $\mathrm{R}^{2}$ coefficient between the two was $>.85$. It therefore measures the ability of the sympathetic nervous system to increase heart rate and vasoconstriction in response to decreased blood pressure. The increase from pre- to post-test while seated suggests that the RMT group had increased sympathetic activity during stress and were better able to maintain blood pressure while upright following RMT. RMT $n=25$; control $n=22$; values are represented as mean \pm standard deviation. 


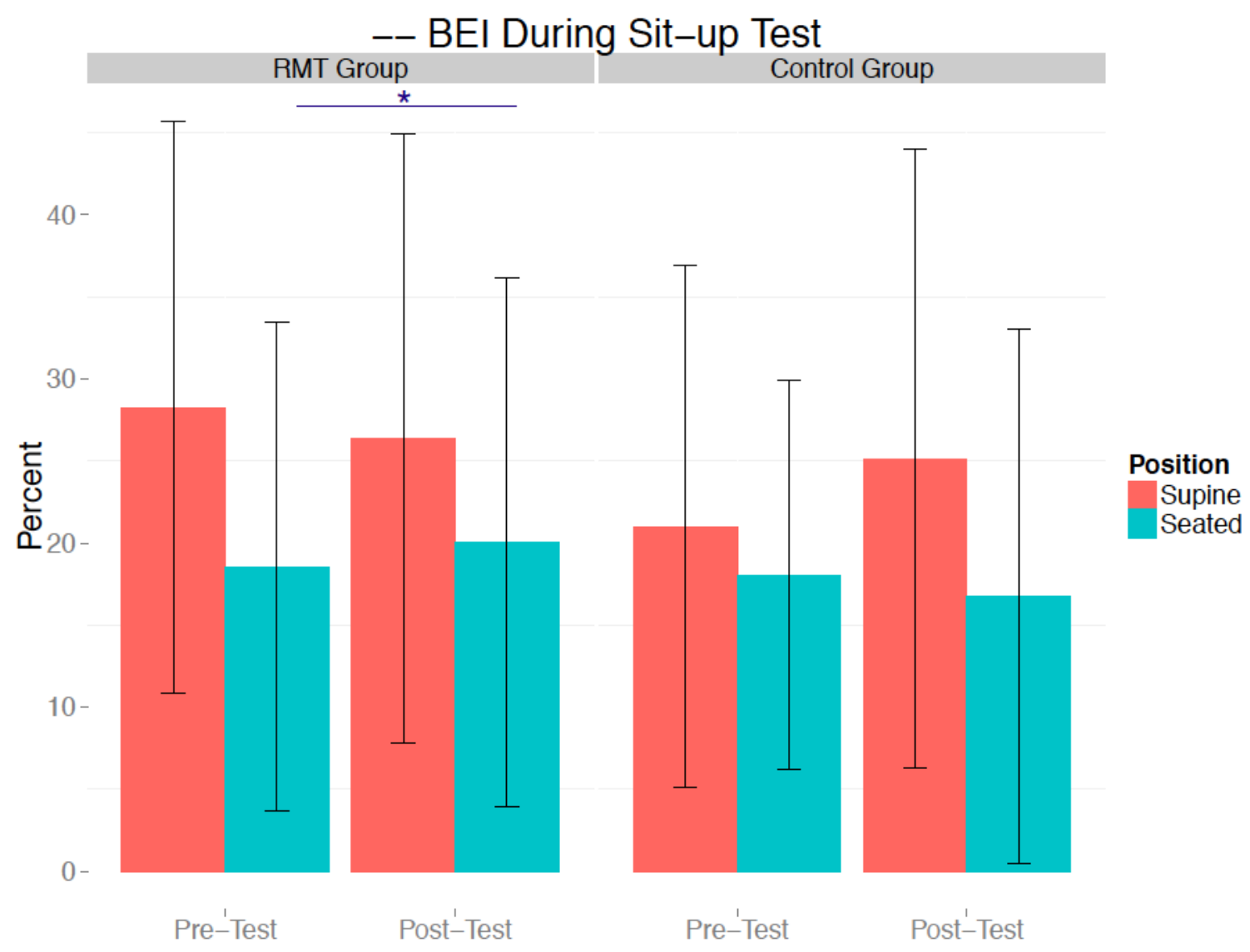

Figure VII-12:Down-down BEI Pre- and Post-RMT

There was a significant increase in down-down baroreceptor effectiveness index (BEI) in the sitting position when comparing the respiratory motor training (RMT) to control groups. Pre-test, the RMT group had a mean down-down BEI of $28 \pm$ 17 percent, which decreased to $26 \pm 19$ percent post-test (model 7-B, $p=.64$ ). Control down-down BEl increased slightly, from $21 \pm 16$ percent pre-test to $25 \pm$ 19 percent post-test. Whilst seated, the RMT group increased from a down-down BEI of $19 \pm 15$ percent pre-test, to $20 \pm 16$ percent, while the control group decreased from $18 \pm 12$ percent pre-test to $16 \pm 16$ percent post-test (model 7-B, $p=.05)$. The down-down BEl is a ratio of baroreceptor engagement, obtained by dividing the number of significant down-down BRS ramp sequences (i.e., the 
sequences of decreasing systolic blood pressure values that cause a decrease in RR interval, and whose $R^{2}$ coefficient is $>.85$ ) into the total number of systolic blood pressure ramps. The down-down BEI measures the role of the sympathetic nervous system in cardiovascular regulation; therefore an increase in the sitting BEl from pre- to post-test in the RMT group indicates an increase in the ability of the sympathetic nervous system to respond to drops in blood pressure by increasing heart rate and vasoconstriction following RMT. RMT $n=25$; control $n$ $=22$; values are represented as mean \pm standard deviation . 

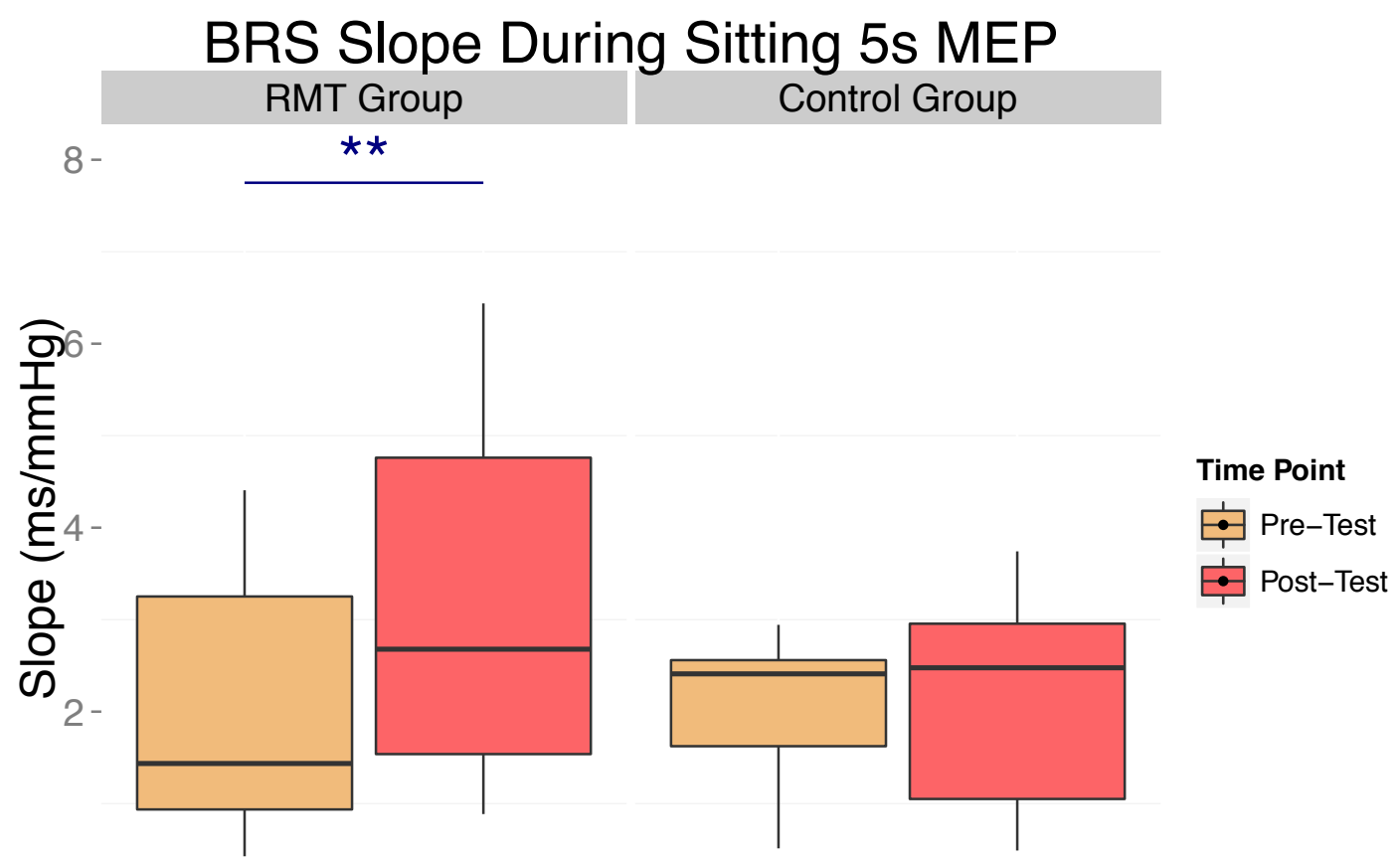

Figure VII-13: Change in BRS During 5s MEP Pre- and Post-RMT

There was a significant increase in the baroreceptor sensitivity (BRS) slope during the 5s MEP in the respiratory motor training (RMT) group when compared to control. In the RMT group, pre-test slope was $2 \pm 2 \mathrm{~ms} / \mathrm{mmHg}$. Following RMT, post-test slope increased to $3 \pm 2 \mathrm{~ms} / \mathrm{mmHg}$ (model 7-B, $\mathrm{p}=.01$ ). The control group pre-test slope was $2 \pm 1 \mathrm{~ms} / \mathrm{mmHg}$, and post-test it remained unchanged at $2 \pm 1 \mathrm{~ms} / \mathrm{mmHg}$. The $5 \mathrm{~s}$ MEP creates a transient drop in blood pressure similar to early phase II of the Valsalva maneuver: intrathoracic pressure increases, decreasing venous return, and engaging the baroreceptors to increase sympathetic efferent activity to increase heart rate. The slope measures the decrease in RR interval (in $\mathrm{ms}$ ) for every $1 \mathrm{mmHg}$ decrease in systolic blood pressure. The significant increase indicates that, following RMT, the baroreceptors were more responsive to drops in SBP and better able to 
overcome rapid drops in BP. In addition, these changes were independent of any changes in the systolic blood pressure or airway pressure generation from pre- to post-test, and as such the change in BRS is unlikely to be the result of an shift of the phase relationship between systolic blood pressure and RR interval. RMT $n=$ 25 ; control $n=22$; values are represented as mean \pm standard deviation. 


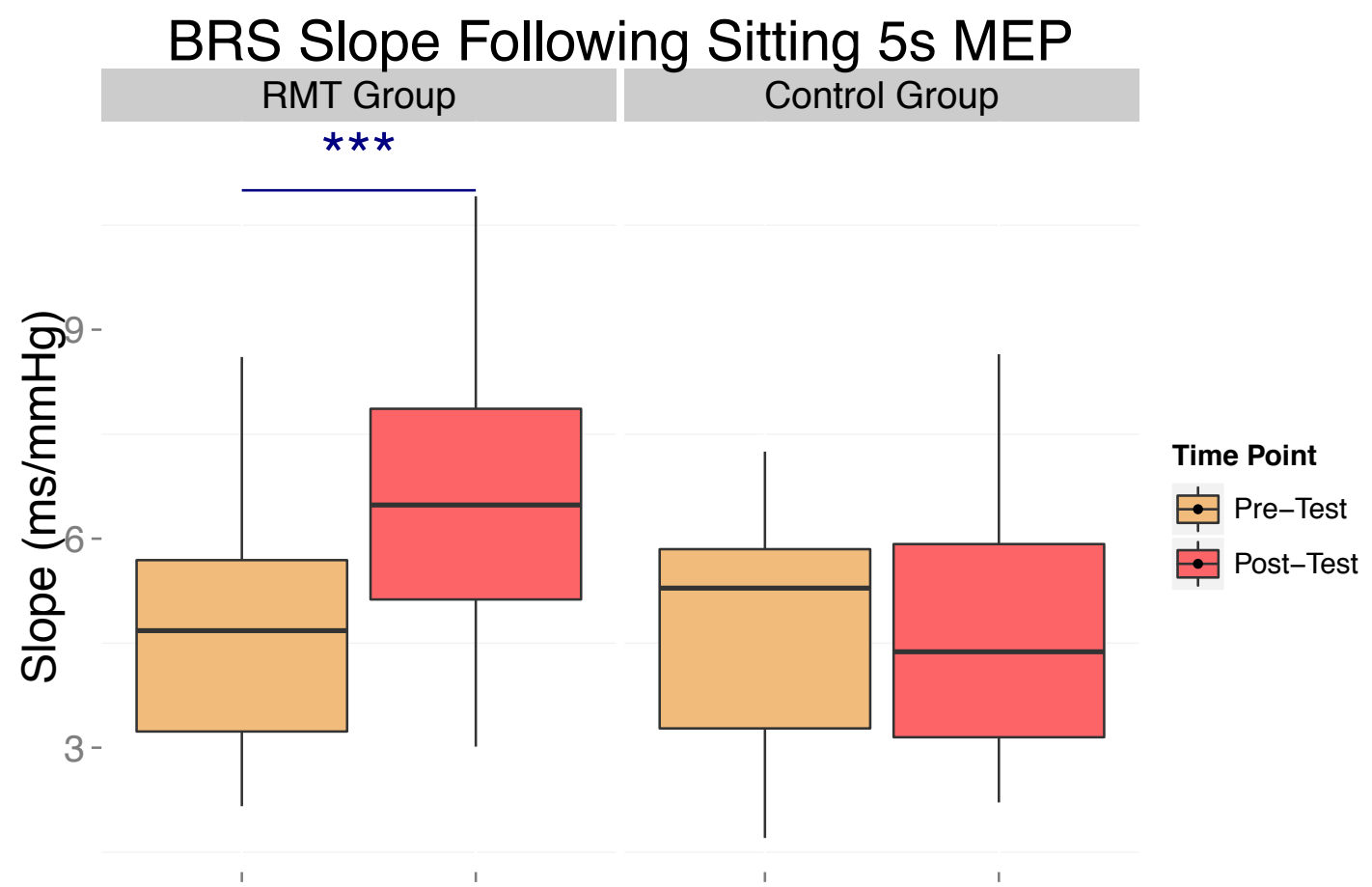

Figure VII-14: Change in BRS Following 5s MEP Pre- and Post-RMT

The baroreceptor sensitivity (BRS) slope of the recovery period following a $5 \mathrm{~s}$ MEP was significantly increased in the respiratory motor training (RMT) group compared to control. In the pre-test, the RMT group had a BRS slope of $5 \pm 2$ $\mathrm{ms} / \mathrm{mmHg}$. Following RMT, the slope increased to $7 \pm 3 \mathrm{~ms} / \mathrm{mmHg}$ (model 2-B, $\mathrm{p}$ $=.001)$. The control group remained unchanged from pre- to post-test at $5 \pm 2$ $\mathrm{ms} / \mathrm{mmHg}$. The recovery period following a $5 \mathrm{~s} M E P$ is similar to that of a Valsalva maneuver (called phase IV): a sudden release of intrathoracic pressure causes blood previously trapped in the vena cava to rapidly enter the heart, creating a rapid increase in systolic blood pressre. The BRS slope measures the vagal response to this rapid increase by quantifying the increase in RR interval (in ms) for every $1 \mathrm{mmHg}$ increase in systolic blood pressure. The pre-test BRS values of 
both groups demonstrate a decreased BRS slope, commonly attributed in SCI to vagal withdrawal. However there was a significant increase in BRS slope in the RMT group, suggesting that there is an increased activity of the cardiovagal loop of the baroreceptor reflex following RMT. In addition, these changes were independent of any changes in the systolic blood pressure or airway pressure generation from pre- to post-test, and as such the change in BRS is unlikely to be the result of an shift of the phase relationship between systolic blood pressure and RR interval. RMT $n=25$; control $n=22$; values are represented as mean \pm standard deviation. 


\section{DISCUSSION}

We found significant increases in the percent-predicted values of FVC and $\mathrm{FEV}_{1}$ and nearly significant increases to airway pressure following RMT that could potentially be significant with more participants. Previously, other researchers have found improvements to pulmonary function in persons with $\mathrm{SCl}$ following respiratory muscle training: Derrickson, et.al.(Derrickson et al 1992), and Van Houtte, et.al. (Van Houtte et al 2008), found significant increases in FVC post-training; Zupan, et.al .(Zupan et al 1997) found significant increases in FEV ${ }_{1}$ post-training; and Gounden (Gounden 1990), Litchke (Litchke et al 2008), Roth(Roth et al 2010), Van Houtte (Van Houtte et al 2008) and their respective colleagues found significant increases in maximum inspiratory pressure generation post-training. Van Houtte and colleagues (Van Houtte et al 2008) were the only researchers that previously found significant increases in more than one pulmonary function outcome: they found both FVC and maximum airway pressure generation increased significantly following training. We also found significant increases in FVC, $\mathrm{FEV}_{1}$, and increases in MEP in the RMT group that were not seen in the control. The discrepancy could be to do the study design: like Van Houtte, our methodology included both resistive inspiratory and expiratory training; others used either resistive inspiratory training (Derrickson et al 1992, Liaw et al 2000, Zupan et al 1997) or resistive expiratory training (Gounden 1990), (Roth et al 2010) alone. Additionally, ours was the only methodology that required participants to train for 45 minutes continuously, five 
days a week; others were 30 minutes or less, 4 days a week (Derrickson et al 1992, Gounden 1990, Liaw et al 2000, Zupan et al 1997). The significant increases we found demonstrate that, in order to maximize benefits of training, the combination of resistive inspiratory and expiratory training and a longer daily duration are both necessary.

The sit-up test, Valsalva maneuver, and 5s MEP all demonstrated that there were significant impairments to cardiovascular regulation in the $\mathrm{SCl}$ groups. However, following RMT, many of these deficits were ameliorated or reversed, with some participants demonstrating outcomes comparable to an NI person. Following RMT, the drop in BP while upright was ameliorated in the RMT group. Of the 12 people in the RMT group that experienced orthostatic hypotension in their screening assessment, only 3 experienced it in the post-test assessment. In the control group there was no alleviation of orthostatic hypotension in those that experienced it in the screening assessment. The changes in SBP and DBP occurred with no significant changes in HR or RRI. The improved regulation thus occurs without an increase in HR, indicating that the reflexes engaged the vasculature to maintain BP better post-RMT, even resulting in an alleviation of the orthostatic hypotension experienced in the pre-test assessment.

Due to the increases in pulmonary function, it seems likely that the cardiopulmonary receptors are more active following RMT: the cardiopulmonary receptors that line the bronchi cause increased efferent traffic to the vasculature during each expiration; with improvements to pulmonary function following RMT, 
it is logical that these receptors would be more active as a result and thus be able to assist during stress.

There were few changes to autonomic balance in the RMT group when compared to control. Post-RMT, there were no significant increases in LF power in the RMT group in neither the supine nor sitting positions. It is therefore unlikely that there was an increase in SNS activity at the SA node following RMT. There was, however, a significant increase in HF power while sitting from pre- to postRMT, indicating an increase in vagal activity following RMT. There were no increases in respiratory frequency, no increases in vagal power while supine, and increased pulmonary function outcomes following RMT. As such, the increased power likely means the cardiopulmonary receptors are be more active during normal respiration post-RMT, which could lead to an increase in vagal activity via the RSA.

Following RMT, there were significant improvements to cardiovascular regulation found in the post-test BRS sequencing. First, the down-down* ramps increased significantly from pre- to post-test in the RMT group, and the slope of those ramp sequences increased as well. This led to a significant increase in down-down BEI while sitting. There was also a significant increase in the up-up* ramps following RMT. This indicates that the baroreceptors are engaged more frequently following RMT during stress, and the magnitude of sympathetic response to stress by the baroreceptors increases following RMT. This could result from training because improved pulmonary function mechanically causes 
increased SBP oscillations, which could potentially reverse some of the arterial stiffening that impairs baroreceptor response.

Finally, there were significant improvements to BRS outcomes obtained by the 5s MEP maneuver: BRS increased significantly in the RMT group both during and following the 5s MEP compared to control. The cardiac loop of the baroreceptor reflex is more active following RMT, which better engaged the baroreceptors, leading to more rapid and greater HR changes in response to increases or decreases in BP. 


\section{CHAPTER VIII:}

\section{CONCLUSIONS}

We are the first to demonstrate the ability of increased pulmonary function to improve cardiovascular regulation in patients with chronic SCI. We found that much of the cardiovascular dysfunction experienced in $\mathrm{SCl}$ is secondary to poor respiratory motor control and not necessarily dependent on level of injury, and as such is reversible. Not only did participants experience improved overall regulation and fewer bouts of $\mathrm{OH}$ following $\mathrm{RMT}$, but the activity of the baoreceptors increased significantly following RMT. It seems, therefore, that the lack of available sympathetic neurons does indeed create regulatory issues in $\mathrm{SCl}$, but many of the cardiovascular problems arise from the poor respiratory pattern, decreased actions of the respiratory pump and cardiopulmonary receptors, and subsequent inactivity of the baroreceptors. This is quite promising because it means that the cardiovascular dysfunctions secondary to $\mathrm{SCl}$ are not permanent, and are quite easily reversible.

First, the orthostatic stress test demonstrated that there was most likely an increase in cardiopulmonary activity following RMT, which lead to fewer bouts of orthostatic hypotension. There were significant increases in HF power, and SBP 
and DBP values while upright, without concurrent changes in respiratory frequency and HR (i.e., post-RMT, the reflexes were able to maintain BP without concurrent increases in HR). HF power while upright is PNS-mediated respiratory oscillations (Wecht et al 2006) This increased vagal power implies that the cardiopulmonary receptors were more active while seated following RMT: with no changes in respiratory frequency, and significant changes in spirometric outcomes and airway pressure generation, there is most likely increased airway pressure oscillation during normal breathing which would increase the activity of the cardiopulmonary receptors that line the bronchi (Burgh Daly 1997, Lee \& Yu 2014). This is the most likely reason for the improved SBP and DBP values postRMT: an improved respiratory pattern increased activity of cardiopulmonary receptors, causing increased efferent traffic to the vasculature, leading to better reflex response post-RMT and fewer bouts of orthostatic hypotension.

We also found significant improvements to baroreceptor activity post-RMT. BRS, as measured by the sequencing method and by the slopes of the Valsalva and 5s MEP, was decreased compared to NI persons. The BRS sequencing demonstrated that the baroreceptors are engaged significantly less in $\mathrm{SCl}$ than $\mathrm{NI}$ persons, indicating that normal oscillations of SBP are smaller in persons with SCl. There was also a decreased BRS measured by the Valsalva maneuver and 5s MEP. Post-RMT, there were improvements to the number of significant ramp sequences (i.e., how frequently the baroreceptors were engaged) and increased $\mathrm{BEI}$, as well as increased BRS slopes measured by the Valsalva and 5s MEP. 
This indicates that, post-RMT, the SBP-oscillations secondary to the respiratory pump increase, which would increase the engagement of the baroreceptors, thereby reversing arterial stiffening common to SCI(Phillips et al 2014): greater SBP oscillations that result from increased activity of the respiratory pump will engage the baroreceptors more frequently during normal activity, which would ultimately demonstrate increases in BRS as measured by the Valsalva and $5 \mathrm{~s}$ MEP maneuvers.

Next is to determine the mechanism for this change. RMT causes greater changes in intrathoracic pressure, which would increase activity of the cardiopulmonary receptors and increase the activity of the baroreceptors by increasing SBP-oscillations. It is quite likely that the continuous pressure oscillations during training reactivated the receptors, especially those that had possibly been unused after SCl. It is also possible that the improved breathing pattern following RMT leads to greater activation of cardiopulmonary receptors during normal respiration; baroreceptors would be increasingly activated with the improved activity of the respiratory pump. Measuring airway pressure generation and activity of the major arteries during normal respiration and during RMT could elucidate the changes to help fine-tune the use of RMT as a therapy to maximize the benefits. In addition to that, there would most likely be a more negative intrathoracic pressure during inspiration following an improved respiratory motor control, which would subsequently lead to an increased venous return and improved activity of the respiratory pump. 
A

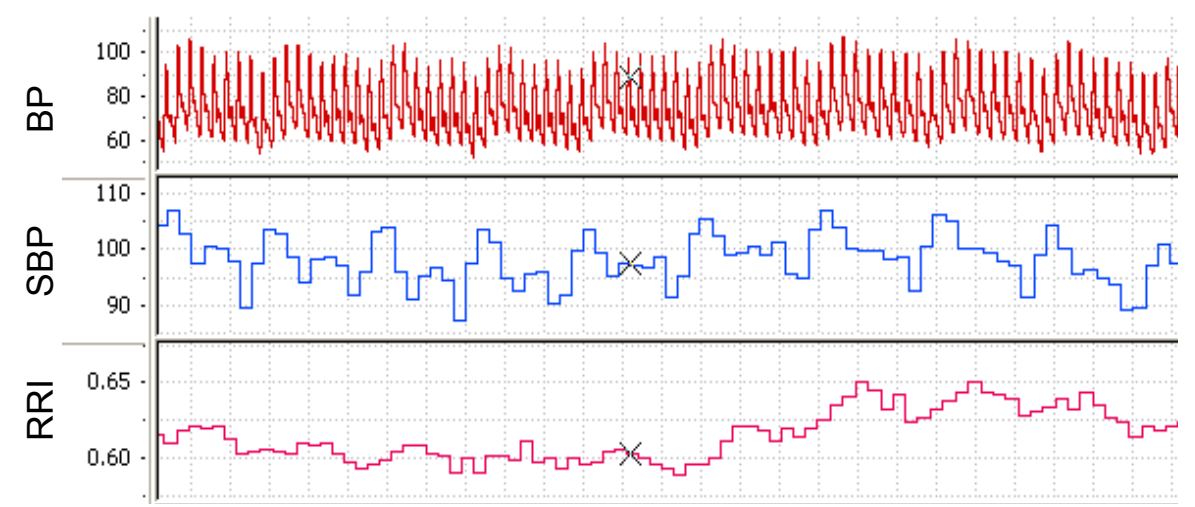

B

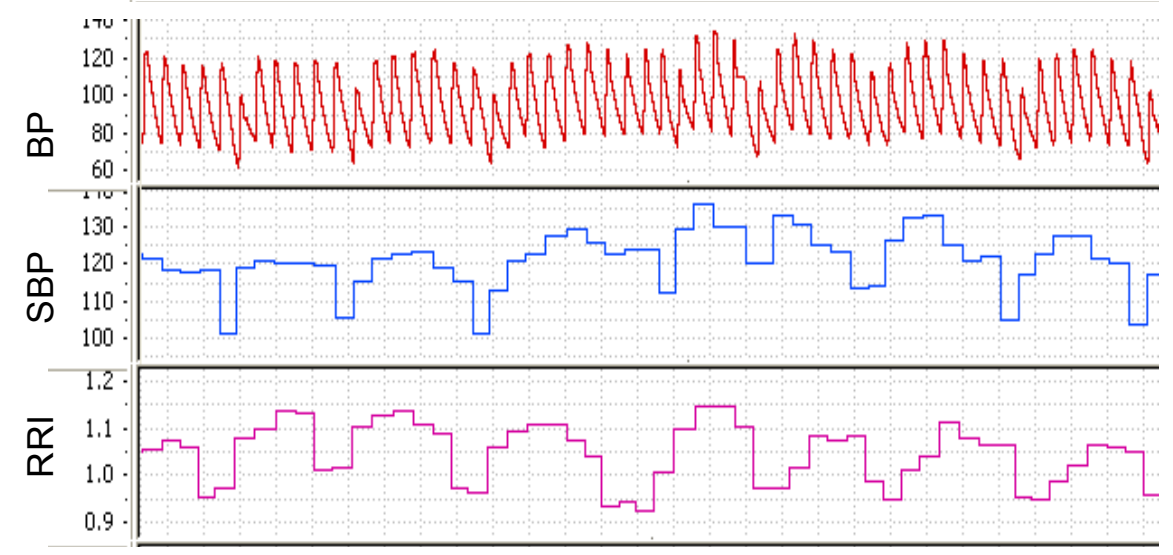

Figure VIII-1: Continuous Hemodynamic Recording during Respiratory Motor

Training

Beat-to-beat recording of blood pressure (BP, mmHg; in red), systolic BP (SBP, $\mathrm{mmHg}$; in blue), and RR interval (RRI, ms; in pink) from sessions 1 (A) and 15 (B) of a C4-A spinal-cord injured (SCl) person during respiratory motor training (RMT). Each block represents $0.20 \mathrm{~ms}$. On day 1 of RMT, there are mechanical oscillations in blood pressure (20 $\mathrm{mmHg}$ from peak to trough) during the training session that are not seen at rest; however, the oscillations of RRI that result are not consistent for every oscillation in SBP, and the total change in RRI is only $0.05 \mathrm{~ms}$. By day $15(\mathrm{~B})$, the mechanical oscillations in SBP are still $20 \mathrm{mmHg}$, yet 
they result in consistent changes in RRI, and the subsequent change in is six times greater $(0.30 \mathrm{~ms})$ than on day 1 . Thus from day 1 to day 15 , there is an increased activity of the receptors following RMT independent of changes in the mechanical oscillations 
Regardless of the mechanism, RMT has great potential to become a universal therapy, and our broad goal is to develop RMT into the standard of care for persons with SCl. There are dramatic benefits following RMT, and it has the ability to target two common dysfunctions with one therapy and potentially decrease the risk factors for developing pulmonary and cardiovascular diseases. Thus, it has great potential to not only improve pulmonary and cardiovascular function in $\mathrm{SCl}$, but decrease the risk of pulmonary and cardiovascular morbidity and mortality.

\section{FUTURE DIRECTIONS}

Our goal is to further study the mechanisms uncovered in this project to assist in our goal of developing RMT into the standard of care for persons with $\mathrm{SCl}$ : we want to solidly identify the mechanisms of cardiovascular impairment that are dependent on respiratory motor control, and then further evaluate the training itself to determine the optimum expiratory and inspiratory loads and training durations in order to maximize the benefits.

First, we want to evaluate arterial stiffness and airway pressure generation in SCI pre- and post-RMT. Arterial stiffness has been identified as a possible culprit of baroreceptor dysfunction in SCI (Phillips et al 2014), so we hope to measure arterial stiffness of the carotid, brachial, and femoral arteries pre- and post-RMT to see if these outcomes change. In addition to that, we want to measure airway pressure generation during eupnea, in order to see if there are any changes from pre- to post-RMT; an increase in tidal volume would 
subsequently increase the activity of the cardiopulmonary receptors (Kobayashi 1998) and allow us to determine changes in cardiopulmonary receptor activity from pre- to post-RMT.

In addition to that, we want to begin cardiovascular recordings during all training sessions, and to continue follow-up assessments after the training has ended. As seen in Figure VIII-1, there is a mechanical oscillation due to RMT that is seen on the first day of training, and the engagement of the receptors thereafter; we want to record SBP and RRI continuously throughout all 20 training sessions for the next cohort of participants in an attempt to determine when the engagement of the receptors occurs. In addition to that, we hope to begin to correlate inspiratory and expiratory loads to the changes, to determine which loads have the greatest and most rapid changes in activity. We also plan on performing follow-up assessments on our participants, in order to determine when these changes start to reverse post-training.

In all, these future directions will allow us to develop RMT into a therapy with a standardized protocol, and quantitative, measurable outcomes posttraining. 


\section{REFERENCES}

1979. Workshop on bronchopulmonary dysplasia. Sponsored by the Division of Lung Diseases. National Heart, Lung, and Blood Institute, National Institutes of Health. The Journal of pediatrics 95: 1-9, 815-920

Almenoff PL, Spungen AM, Lesser M, Bauman WA. 1995. Pulmonary function survey in spinal cord injury: influences of smoking and level and completeness of injury. Lung 173: 297-306

Anthony Cahill PD, Heidi Fredine M, Luciana Zilberman M. 2009. Prevalence of Paralysis Including Spinal Cord Injuries in the United States, 2008, University of New Mexico Center for Development and Disability

Arnold JM, Feng QP, Delaney GA, Teasell RW. 1995. Autonomic dysreflexia in tetraplegic patients: evidence for alpha-adrenoceptor hyper-responsiveness. Clinical autonomic research : official journal of the Clinical Autonomic Research Society 5: $267-70$

Aslan SC, Chopra MK, McKay WB, Folz RJ, Ovechkin AV. 2013. Evaluation of respiratory muscle activation using respiratory motor control assessment (RMCA) 
in individuals with chronic spinal cord injury. Journal of visualized experiments : JoVE

Bach JR. 2012. Noninvasive respiratory management of high level spinal cord injury. The journal of spinal cord medicine 35: 72-80

Baselli G, Cerutti S, Civardi S, Liberati D, Lombardi F, et al. 1986. Spectral and crossspectral analysis of heart rate and arterial blood pressure variability signals. Computers and biomedical research, an international journal 19: 520-34

Berne RMKBMSBA. 2010. Berne \& Levy physiology. Philadelphia, PA: Mosby/Elsevier.

Bertinieri G, di Rienzo M, Cavallazzi A, Ferrari AU, Pedotti A, Mancia G. 1985. A new approach to analysis of the arterial baroreflex. Journal of hypertension. Supplement : official journal of the International Society of Hypertension 3: S7981

Blomqvist CG. 2011. Cardiovascular Adjustments to Gravitational Stress In Comprehensive Physiology: The Cardiovascular System, pp. 1025-63: John Wiley \& Sons, Inc

Bluvshtein V, Korczyn AD, Akselrod S, Pinhas I, Gelernter I, Catz A. 2011. Hemodynamic responses to head-up tilt after spinal cord injury support a role for the mid-thoracic spinal cord in cardiovascular regulation. Spinal cord 49: 251-6 
Boron WF, Boulpaep EL. 2009. Medical physiology : a cellular and molecular approach. Philadelphia, PA: Saunders/Elsevier. xii, 1337 p. pp.

Brock JA, Yeoh M, McLachlan EM. 2006. Enhanced neurally evoked responses and inhibition of norepinephrine reuptake in rat mesenteric arteries after spinal transection. American journal of physiology. Heart and circulatory physiology 290: H398-405

Brown A, Weaver LC. 2012. The dark side of neuroplasticity. Experimental neurology 235: $133-41$

Brown R, DiMarco AF, Hoit JD, Garshick E. 2006. Respiratory dysfunction and management in spinal cord injury. Respiratory care 51: 853-68;discussion 69-70

Burgh Daly Md. 1997. Peripheral arterial chemoreceptors and respiratory-cardiovascular integration. Oxford

New York: Clarendon Press, Oxford University Press. xvi, 739 p. pp.

Casha S, Christie S. 2011. A systematic review of intensive cardiopulmonary management after spinal cord injury. Journal of neurotrauma 28: 1479-95

Castiglioni P, Di Rienzo M, Veicsteinas A, Parati G, Merati G. 2007. Mechanisms of blood pressure and heart rate variability: an insight from low-level paraplegia. American journal of physiology. Regulatory, integrative and comparative physiology 292: R1502-9 
Chiappa GR, Roseguini BT, Vieira PJ, Alves CN, Tavares A, et al. 2008. Inspiratory muscle training improves blood flow to resting and exercising limbs in patients with chronic heart failure. Journal of the American College of Cardiology 51: $1663-71$

Claydon VE, Krassioukov AV. 2008. Clinical correlates of frequency analyses of cardiovascular control after spinal cord injury. American journal of physiology. Heart and circulatory physiology 294: H668-78

Cohen-Adad J, El Mendili MM, Lehericy S, Pradat PF, Blancho S, et al. 2011. Demyelination and degeneration in the injured human spinal cord detected with diffusion and magnetization transfer MRI. Neurolmage 55: 1024-33

Committee M, Burns S, Biering-Sorensen F, Donovan W, Graves DE, et al. 2012. International standards for neurological classification of spinal cord injury, revised 2011. Topics in spinal cord injury rehabilitation 18: 85-99

Conforti L, Gilley J, Coleman MP. 2014. Wallerian degeneration: an emerging axon death pathway linking injury and disease. Nature reviews. Neuroscience 15: 394409

Cotton BA, Pryor JP, Chinwalla I, Wiebe DJ, Reilly PM, Schwab CW. 2005. Respiratory complications and mortality risk associated with thoracic spine injury. The Journal of trauma 59: 1400-7; discussion 07-9 
Cronenwett J, Johnston KW. 2014. Rutherford's Vascular surgery. Philadelphia:

Saunders.

Crossman AR, Neary D. 2010. Neuroanatomy : an illustrated colour text. Edinburgh ; New York: Churchill Livingstone. ix, 188 p. pp.

De Troyer A, Estenne M, Vincken W. 1986. Rib cage motion and muscle use in high tetraplegics. The American review of respiratory disease 133: 1115-9

De Troyer A, Heilporn A. 1980. Respiratory mechanics in quadriplegia. The respiratory function of the intercostal muscles. The American review of respiratory disease 122: $591-600$

deBoer RW, Karemaker JM, Strackee J. 1987. Hemodynamic fluctuations and baroreflex sensitivity in humans: a beat-to-beat model. The American journal of physiology 253: H680-9

Derrickson J, Ciesla N, Simpson N, Imle PC. 1992. A comparison of two breathing exercise programs for patients with quadriplegia. Physical therapy 72: 763-9

Estenne M, De Troyer A. 1986. The effects of tetraplegia on chest wall statics. The American review of respiratory disease 134: 121-4

Estenne M, Gorini M. 1992. Action of the diaphragm during cough in tetraplegic subjects. Journal of applied physiology 72: 1074-80 
Fishburn MJ, Marino RJ, Ditunno JF, Jr. 1990. Atelectasis and pneumonia in acute spinal cord injury. Archives of physical medicine and rehabilitation 71: 197-200

Forster Hv WJMPLGHMRBR. 2004. Eupneic respiratory rhythm in awake goats is dependent on an intact pre-Bötzinger complex. Advances in experimental medicine and biology 551: 107-13

Fox MH, Krahn GL, Sinclair LB, Cahill A. 2015. Using the international classification of functioning, disability and health to expand understanding of paralysis in the United States through improved surveillance. Disability and health journal

Frankel H, Mathias CJ. 1992. The Cardiovascular System in Tetraplegia and Paraplegia In Handbook of Clinical Neurology: Spinal Cord Trauma, ed. PJ Vinken, GW Bruyn, HL Klawans, HL Frankel, pp. xv, 586 p. Amsterdam ; New York: Elsevier

Frisbie JH. 2005. Breathing and the support of blood pressure after spinal cord injury. Spinal cord 43: 406-7

Fuller DD, Golder FJ, Olson EB, Jr., Mitchell GS. 2006. Recovery of phrenic activity and ventilation after cervical spinal hemisection in rats. J Appl Physiol 100: 800-6

Furlan JC, Fehlings MG, Halliday W, Krassioukov AV. 2003. Autonomic dysreflexia associated with intramedullary astrocytoma of the spinal cord. The lancet oncology 4: 574-5 
Galeiras Vazquez R, Rascado Sedes P, Mourelo Farina M, Montoto Marques A, Ferreiro Velasco ME. 2013. Respiratory management in the patient with spinal cord injury. Biomed Res Int 2013: 168757

Garshick E, Kelley A, Cohen SA, Garrison A, Tun CG, et al. 2005. A prospective assessment of mortality in chronic spinal cord injury. Spinal cord 43: 408-16

Gillis DJ, Wouda M, Hjeltnes N. 2008. Non-pharmacological management of orthostatic hypotension after spinal cord injury: a critical review of the literature. Spinal cord 46: $652-9$

Gondim FA, Lopes AC, Jr., Oliveira GR, Rodrigues CL, Leal PR, et al. 2004. Cardiovascular control after spinal cord injury. Current vascular pharmacology 2: $71-9$

Gorlin R, Knowles JH, Storey CF. 1957. The Valsalva maneuver as a test of cardiac function; pathologic physiology and clinical significance. The American journal of medicine 22: 197-212

Gounden P. 1990. Progressive resistive loading on accessory expiratory muscles in tetraplegia. S Afr J Physiother 42: 4-12

Gounden P. 1997. Static respiratory pressures in patients with post-traumatic tetraplegia. Spinal cord 35: 43-7 
Grimm DR, Almenoff PL, Bauman WA, De Meersman RE. 1998. Baroreceptor sensitivity response to phase IV of the Valsalva maneuver in spinal cord injury. Clinical autonomic research : official journal of the Clinical Autonomic Research Society 8: $111-8$

Grimm DR, De Meersman RE, Almenoff PL, Spungen AM, Bauman WA. 1997. Sympathovagal balance of the heart in subjects with spinal cord injury. The American journal of physiology 272: $\mathrm{H} 835-42$

Haines DE. 2006. Fundamental neuroscience. Philadelphia: Churchill Livingstone. 567 p. pp.

Hall JEGAC. 2011. Guyton and Hall textbook of medical physiology. Philadelphia, Pa.: Saunders/Elsevier.

Hart EC, Joyner MJ, Wallin BG, Karlsson T, Curry TB, Charkoudian N. 2010. Baroreflex control of muscle sympathetic nerve activity: a nonpharmacological measure of baroreflex sensitivity. American journal of physiology. Heart and circulatory physiology 298: H816-22

Hayano J, Yasuma F. 2003. Hypothesis: respiratory sinus arrhythmia is an intrinsic resting function of cardiopulmonary system. Cardiovascular research 58: 1-9

Houtman S, Oeseburg B, Hopman MT. 1999. Non-invasive assessment of autonomic nervous system integrity in able-bodied and spinal cord-injured individuals. 
Clinical autonomic research : official journal of the Clinical Autonomic Research Society 9: 115-22

Houtman S, Oeseburg B, Hughson RL, Hopman MT. 2000. Sympathetic nervous system activity and cardiovascular homeostatis during head-up tilt in patients with spinal cord injuries. Clinical autonomic research : official journal of the Clinical Autonomic Research Society 10: 207-12

Iglewicz B, Hoaglin DC. 1993. How to detect and handle outliers. Milwaukee, Wis.: ASQC Quality Press. ix, 87 p. pp.

Inoue K, Miyake S, Kumashiro M, Ogata H, Yoshimura O. 1990. Power spectral analysis of heart rate variability in traumatic quadriplegic humans. The American journal of physiology 258: H1722-6

Inoue K, Ogata H, Hayano J, Miyake S, Kamada T, et al. 1995. Assessment of autonomic function in traumatic quadriplegic and paraplegic patients by spectral analysis of heart rate variability. Journal of the autonomic nervous system 54 : 225-34

Kamiya A, Kawada T, Shimizu S, Sugimachi M. 2011. Closed-loop spontaneous baroreflex transfer function is inappropriate for system identification of neural arc but partly accurate for peripheral arc: predictability analysis. The Journal of physiology 589: 1769-90 
Kerschensteiner M, Schwab ME, Lichtman JW, Misgeld T. 2005. In vivo imaging of axonal degeneration and regeneration in the injured spinal cord. Nature medicine 11: $572-7$

Kobayashi H. 1998. Normalization of Respiratory Sinus Arrhythmia by Factoring in Tidal Volume. Applied Human Science 17: 207-13

Krassioukov A. 2006. Which pathways must be spared in the injured human spinal cord to retain cardiovascular control? Progress in brain research 152: 39-47

Krassioukov AV, Karlsson AK, Wecht JM, Wuermser LA, Mathias CJ, Marino RJ. 2007. Assessment of autonomic dysfunction following spinal cord injury: rationale for additions to International Standards for Neurological Assessment. Journal of rehabilitation research and development 44: 103-12

Krause JS, Saunders LL. 2011. Health, secondary conditions, and life expectancy after spinal cord injury. Archives of physical medicine and rehabilitation 92: 1770-5

Lee LY, Yu J. 2014. Sensory nerves in lung and airways. Comprehensive Physiology 4: 287-324

Legramante JM, Raimondi G, Massaro M, lellamo F. 2001. Positive and negative feedback mechanisms in the neural regulation of cardiovascular function in healthy and spinal cord-injured humans. Circulation 103: 1250-5 
Lewis ME, Al-Khalidi AH, Bonser RS, Clutton-Brock T, Morton D, et al. 2001. Vagus nerve stimulation decreases left ventricular contractility in vivo in the human and pig heart. The Journal of physiology 534: 547-52

Liaw MY, Lin MC, Cheng PT, Wong MK, Tang FT. 2000. Resistive inspiratory muscle training: its effectiveness in patients with acute complete cervical cord injury. Archives of physical medicine and rehabilitation 81: 752-6

Linn WS, Adkins RH, Gong H, Jr., Waters RL. 2000. Pulmonary function in chronic spinal cord injury: a cross-sectional survey of 222 southern California adult outpatients. Archives of physical medicine and rehabilitation 81: 757-63

Litchke LG, Russian CJ, Lloyd LK, Schmidt EA, Price L, Walker JL. 2008. Effects of respiratory resistance training with a concurrent flow device on wheelchair athletes. The journal of spinal cord medicine 31: 65-71

Lumb AB, Nunn JF. 2005. Nunn's applied respiratory physiology. Edinburgh ; Philadelphia: Elsevier Butterworth Heinemann. xiii, 501 p. pp.

Maier IC, Schwab ME. 2006. Sprouting, regeneration and circuit formation in the injured spinal cord: factors and activity. Philosophical transactions of the Royal Society of London. Series B, Biological sciences 361: 1611-34

Mateus SR, Beraldo PS, Horan TA. 2007. Maximal static mouth respiratory pressure in spinal cord injured patients: correlation with motor level. Spinal cord 45: 569-75 
Mathias CJ, Christensen NJ, Frankel HL, Spalding JM. 1979. Cardiovascular control in recently injured tetraplegics in spinal shock. The Quarterly journal of medicine 48: $273-87$

McGillivray CF, Hitzig SL, Craven BC, Tonack MI, Krassioukov AV. 2009. Evaluating knowledge of autonomic dysreflexia among individuals with spinal cord injury and their families. The journal of spinal cord medicine 32: 54-62

McLachlan EM, Brock JA. 2006. Adaptations of peripheral vasoconstrictor pathways after spinal cord injury. Progress in brain research 152: 289-97

Middleton JW, Dayton A, Walsh J, Rutkowski SB, Leong G, Duong S. 2012. Life expectancy after spinal cord injury: a 50-year study. Spinal cord

Miller MR, Hankinson J, Brusasco V, Burgos F, Casaburi R, et al. 2005. Standardisation of spirometry. The European respiratory journal : official journal of the European Society for Clinical Respiratory Physiology 26: 319-38

Mills PB, Fung CK, Travlos A, Krassioukov A. 2015. Nonpharmacologic Management of Orthostatic Hypotension: A Systematic Review. Archives of physical medicine and rehabilitation 96: 366-75.e6

Munakata M, Kameyama J, Nunokawa T, Ito N, Yoshinaga K. 2001. Altered Mayer wave and baroreflex profiles in high spinal cord injury. American journal of hypertension 14: 141-8 
NSCISC. 2014. The 2014 Annual Statistical Report for the Spinal Cord Injury Model Systems, University of Alabama, Birmingham

O'Leary DD, Kimmerly DS, Cechetto AD, Shoemaker JK. 2003. Differential effect of head-up tilt on cardiovagal and sympathetic baroreflex sensitivity in humans. Experimental physiology 88: 769-74

Ori Z, Monir G, Weiss J, Sayhouni X, Singer DH. 1992. Heart rate variability. Frequency domain analysis. Cardiology clinics 10: 499-537

Ovechkin A, Vitaz T, de Paleville DT, Aslan S, McKay W. 2010. Evaluation of respiratory muscle activation in individuals with chronic spinal cord injury. Respiratory physiology \& neurobiology 173: 171-8

Palmero HA, Caeiro TF, losa DJ, Bas J. 1981. Baroreceptor reflex sensitivity index derived from Phase 4 of te Valsalva maneuver. Hypertension 3: II-134-7

Parati G, Di Rienzo M, Mancia G. 2000. How to measure baroreflex sensitivity: from the cardiovascular laboratory to daily life. Journal of hypertension 18: 7-19

Persson PB, Kirchheim HR. 1991. Baroreceptor reflexes : integrative functions and clinical aspects. Berlin ; New York: Springer-Verlag. x, 322 p. pp. 
Phillips AA, Krassioukov AV. 2015. Contemporary Cardiovascular Concerns after Spinal Cord Injury: Mechanisms, Maladaptations \& Management. Journal of neurotrauma

Phillips AA, Krassioukov AV, Ainslie PN, Cote AT, Warburton DE. 2014. Increased Central Arterial Stiffness Explains Baroreflex Dysfunction in Spinal Cord Injury. Journal of neurotrauma

Popa C, Popa F, Grigorean VT, Onose G, Sandu AM, et al. 2010. Vascular dysfunctions following spinal cord injury. Journal of medicine and life 3: 275-85

Porth CJ, Bamrah VS, Tristani FE, Smith JJ. 1984. The Valsalva maneuver: mechanisms and clinical implications. Heart \& lung : the journal of critical care 13: $507-18$

Previnaire JG, Mathias CJ, EI Masri W, Soler JM, Leclercq V, Denys P. 2010. The isolated sympathetic spinal cord: Cardiovascular and sudomotor assessment in spinal cord injury patients: A literature survey. Annals of physical and rehabilitation medicine 53: 520-32

Previnaire JG, Soler JM, Leclercq V, Denys P. 2012. Severity of autonomic dysfunction in patients with complete spinal cord injury. Clinical autonomic research : official journal of the Clinical Autonomic Research Society 22: 9-15 
Roth EJ, Stenson KW, Powley S, Oken J, Primack S, et al. 2010. Expiratory muscle training in spinal cord injury: a randomized controlled trial. Archives of physical medicine and rehabilitation 91: 857-61

Routal RV, Pal GP. 1999. Location of the phrenic nucleus in the human spinal cord. Journal of anatomy 195 ( Pt 4): 617-21

Sagawa K. 2011. Baroreflex Control of Systemic Arterial Pressure and Vascular Bed In Comprehensive Physiology, pp. 453-96: John Wiley \& Sons, Inc.

Sahota IS, Ravensbergen HR, McGrath MS, Claydon VE. 2012. Cerebrovascular responses to orthostatic stress after spinal cord injury. Journal of neurotrauma 29: $2446-56$

Sala-Mercado JA, Moslehpour M, Hammond RL, Ichinose M, Chen X, et al. 2014. Stimulation of the cardiopulmonary baroreflex enhances ventricular contractility in awake dogs: a mathematical analysis study. American journal of physiology. Regulatory, integrative and comparative physiology 307: R455-64

Scher A, O'Leary D, Sheriff D. 1991. Arterial Baroreceptor Regulation of Peripheral Resistance and of Cardiac Performance In Baroreceptor Reflexes, ed. PB Persson, HR Kirchheim, pp. 75-125: Springer Berlin Heidelberg 
Schilero GJ, Spungen AM, Bauman WA, Radulovic M, Lesser M. 2009. Pulmonary function and spinal cord injury. Respiratory physiology \& neurobiology 166: 12941

Segal N, Yannopoulos D, Truchot J, Laribi S, Plaisance P, Convertino VA. 2013. [Improving vital organs perfusion by the respiratory pump: physiology and clinical use]. Annales francaises d'anesthesie et de reanimation 32: 572-9

Smart NA, Giallauria F, Dieberg G. 2012. Efficacy of inspiratory muscle training in chronic heart failure patients: A systematic review and meta-analysis. International journal of cardiology

Smith JEHBKRDFJ. 1991. Pre-Botzinger complex: a brainstem region that may generate respiratory rhythm in mammals. Science Science 254: 726-29

Smith SA, Stallard TJ, Salih MM, Littler WA. 1987. Can sinoaortic baroreceptor heart rate reflex sensitivity be determined from phase IV of the Valsalva manoeuvre? Cardiovascular research 21: 422-7

Spronck B, Megens RT, Reesink KD, Delhaas T. 2014. Three-dimensional vascular smooth muscle orientation as quantitatively assessed by multiphoton microscopy: mouse carotid arteries do show a helix. Conference proceedings : ... Annual International Conference of the IEEE Engineering in Medicine and Biology Society. IEEE Engineering in Medicine and Biology Society. Conference 2014: $202-5$ 
Steward O, Zheng B, Tessier-Lavigne M. 2003. False resurrections: distinguishing regenerated from spared axons in the injured central nervous system. The Journal of comparative neurology 459: 1-8

Stolzmann KL, Gagnon DR, Brown R, Tun CG, Garshick E. 2008. Longitudinal change in FEV1 and FVC in chronic spinal cord injury. American journal of respiratory and critical care medicine 177: 781-6

Stolzmann KL, Gagnon DR, Brown R, Tun CG, Garshick E. 2010. Risk factors for chest illness in chronic spinal cord injury: a prospective study. American journal of physical medicine \& rehabilitation / Association of Academic Physiatrists 89: 57683

Sutbeyaz ST, Koseoglu F, Inan L, Coskun O. 2010. Respiratory muscle training improves cardiopulmonary function and exercise tolerance in subjects with subacute stroke: a randomized controlled trial. Clinical rehabilitation 24: 240-50

Tamplin J, Berlowitz DJ. 2014. A systematic review and meta-analysis of the effects of respiratory muscle training on pulmonary function in tetraplegia. Spinal cord 52: $175-80$

Teasell RW, Arnold JM, Krassioukov A, Delaney GA. 2000. Cardiovascular consequences of loss of supraspinal control of the sympathetic nervous system after spinal cord injury. Archives of physical medicine and rehabilitation 81: 50616 
Terson de Paleville DG, McKay WB, Folz RJ, Ovechkin AV. 2011. Respiratory Motor Control Disrupted by Spinal Cord Injury: Mechanisms, Evaluation, and Restoration. Translational stroke research 2: 463-73

Tollefsen E, Fondenes O. 2012. Respiratory complications associated with spinal cord injury. Tidsskrift for den Norske laegeforening : tidsskrift for praktisk medicin, ny raekke 132: 1111-4

van Gestel AJR, Steier J. 2010. Autonomic dysfunction in patients with chronic obstructive pulmonary disease (COPD). Journal of Thoracic Disease 2: 215-22

Van Houtte S, Vanlandewijck Y, Gosselink R. 2006. Respiratory muscle training in persons with spinal cord injury: a systematic review. Respiratory medicine 100: $1886-95$

Van Houtte S, Vanlandewijck Y, Kiekens C, Spengler CM, Gosselink R. 2008. Patients with acute spinal cord injury benefit from normocapnic hyperpnoea training. Journal of rehabilitation medicine : official journal of the UEMS European Board of Physical and Rehabilitation Medicine 40: 119-25

Vincent J-L, Abraham E, Kochanek P, Moore F. 2011. Textbook of Critical Care. Philadelphia: W.B. Saunders. 1744 pp. 
Vinit S, Kastner A. 2009. Descending bulbospinal pathways and recovery of respiratory motor function following spinal cord injury. Respiratory physiology \& neurobiology 169: $115-22$

Vittinghoff E. 2012. Regression methods in biostatistics : linear, logistic, survival, and repeated measures models. New York: Springer. xx, 509 p. pp.

Vogel ER, Sandroni P, Low PA. 2005. Blood pressure recovery from Valsalva maneuver in patients with autonomic failure. Neurology 65: 1533-7

Wadsworth BM, Haines TP, Cornwell PL, Paratz JD. 2009. Abdominal binder use in people with spinal cord injuries: a systematic review and meta-analysis. Spinal cord 47: 274-85

Walters BC, Hadley MN, Hurlbert RJ, Aarabi B, Dhall SS, et al. 2013. Guidelines for the management of acute cervical spine and spinal cord injuries: 2013 update. Neurosurgery 60 Suppl 1: 82-91

Wecht JM, De Meersman RE, Weir JP, Spungen AM, Bauman WA. 2003. Cardiac autonomic responses to progressive head-up tilt in individuals with paraplegia. Clinical autonomic research : official journal of the Clinical Autonomic Research Society 13: 433-8 
Wecht JM, Weir JP, Bauman WA. 2006. Blunted heart rate response to vagal withdrawal in persons with tetraplegia. Clinical autonomic research : official journal of the Clinical Autonomic Research Society 16: 378-83

West CR, Alyahya A, Laher I, Krassioukov A. 2013. Peripheral vascular function in spinal cord injury: a systematic review. Spinal cord 51: 10-9

West CR, Mills P, Krassioukov AV. 2012. Influence of the neurological level of spinal cord injury on cardiovascular outcomes in humans: a meta-analysis. Spinal cord

Zimmer MB, Nantwi K, Goshgarian HG. 2007. Effect of spinal cord injury on the respiratory system: basic research and current clinical treatment options. The journal of spinal cord medicine 30: 319-30

Zollei E, Paprika D, Rudas L. 2003. Measures of cardiovascular autonomic regulation derived from spontaneous methods and the Valsalva maneuver. Autonomic neuroscience : basic \& clinical 103: 100-5

Zupan A, Savrin R, Erjavec T, Kralj A, Karcnik T, et al. 1997. Effects of respiratory muscle training and electrical stimulation of abdominal muscles on respiratory capabilities in tetraplegic patients. Spinal cord 35: 540-5 


\section{APPENDIX A:}

\section{ABBREVIATIONS}

BP: Blood Pressure

BRS: Baroreceptor reflex sensitivity

DBP: Diastolic blood pressure

HF: High Frequency

HR: Heart rate

HRV: Heart Rate Variability

LF: Low Frequency

NI: Non-injured

PNS: Parasympathetic Nervous System

RMC: Respiratory Motor Control

RMCA: Respiratory Motor Control Assessment

RMT: Respiratory Motor Training

RRI: R-R Interval

SBP: Systolic Blood Pressure

SCI: Spinal Cord Injury/Injured

SNS: Sympathetic Nervous System 


\section{APPENDIX B:}

\section{INFORMED CONSENTS}




\title{
SUBJECT INFORMED CONSENT DOCUMENT AND RESEARCH AUTHORIZATION
}

UofL Institutional Review Boards IRB NUMBER: 11.0043

\section{Respiratory Motor Control And Blood Pressure Regulation After Spinal Cord Injury}

\author{
Investigator(s) name \& address:
}

Alexander Ovechkin, $\mathrm{PhD}$

Frazier Rehab Institute,

220 Abraham Flexner Way, Louisville, KY 40202

Site(s) where study is to be conducted: Jewish Hospital/Frazier Rehab Institute

Phone number for subjects to call for questions: (502) 581-8675

\section{Introduction and Background Information}

You are invited to take part in a research study because we are interested in understanding how lungs, heart and blood vessels works in patients with spinal cord injury. The study is being conducted under the direction of Alexander Ovechkin, PhD. Darryl Kaelin, MD, Sarah Wagers, MD, and Megan Nelson, MD are physicians designated to oversee the patient-related aspects of this study. These patient-related aspects include medical evaluations prior to inclusion and attending to patient needs during the research study. Approximately 50 subjects (at least 18 years of age) will be invited to participate over a five-year period totally. Your participation in this study will last for 7-8 months. You might be asked to be tested repeatedly during the 2-year period after completion period of the study. This study will take place at the Frazier Rehab Institute.

\section{Purpose}

The purpose of this study is to investigate whether the improvement in muscles we use to breathe can also change the way our body regulates blood pressure inside the vessels in people with spinal cord injury. The results of this study may aid in the development of treatments to help individuals with spinal cord injuries that also have low arterial blood pressure.

\section{Procedures}

\section{Screening:}

To determine your eligibility for this study, you have to participate in a screening respiratory and cardiovascular function tests (described below). If inclusion criteria are met, you will be asked to see the study physician, who will perform a general physical examination. Also, he/she or a physical therapist will perform an ASIA (American Spinal Injury Association) examination, which is used to grade your level and degree of spinal cord injury. According to results of this screening process, the final decision about your eligibility for this study will be made.

If you volunteer to participate in this experimental study, and sign this consent form, we will ask you to participate in a series of tests. You will be asked to participate in a trained or non-trained group of participants. In the trained group, after initial tests, you will be assigned to receive the Respiratory Muscle Training (RMT, described below) consisting of approximately 20 training sessions that will last about 1 hour each. You will be tested immediately after this training period and repeatedly during the 6-month follow-up period (up to 6 sets of testing). In the non-trained group, you will undergo the same procedures except training. If you are assigned to be in the non-trained group, at the end of your term as a non-trained participant, you can be offered the RMT with after-training testing. You will not be 
Respiratory motor control and blood pressure regulation after spinal cord injury

Alexander Ovechkin, $\mathrm{PhD}$ required to stay in the hospital. Additionally, you might be asked to be tested during the 2-year period following the training (up to another 3 sets of testing).

Tests:

The standard measurements (video and audio recorded for data analysis and presentation) of lung, heart and blood vessel function will be performed before, and after the training program (see appendix).

You will be asked to answer the questions about your physical health and related activities by filling out the standard questionnaire forms. This will take no more than 30 minutes.

You have the right to refuse to answer any questions that you may not wish to answer and to refuse any test. Our personnel will assist you during training and tests.

- If you are assigned to receive the RMT, we will ask you to breathe through a special device which requires you to use additional efforts to pass the air through (described below).

\section{Respiratory Muscle Training (RMT):}

Each training session will last about 45-60 min and will occur five times weekly during one month. During the RMT sessions, you will remain in your personal wheelchair. You will be asked to breath through a special device with regulated resistance to breathing air. In the 20 sessions starting from the lowest resistance, the goal will be to train the muscles you use to breathe by slowly increasing this resistance. You will perform six work sets, 5 minutes in duration, separated by rest intervals lasting 1-3 minutes. If you should feel tired or need a break during the session you can take a break at any time. Ideally, the RMT sessions will take place on site in our Laboratory at Frazier Rehab in Room 1102. However, in certain situations we may allow the RMT to be done from your home. If this situation should occur, we would invite you to Frazier Rehab to be instructed on how to use the RMT device and then monitor you from your homes via a web camera. Laboratory personnel would train you to follow the proper protocol, effectively use the portable blood pressure monitor, and teach you how to use standard web camera software. We would supply you with all of the necessary equipment, except for a personal computer. The items supplied would include: a web camera, web camera software, the RMT device, and a portable blood pressure monitor. During each training session, you would be monitored and coached by laboratory personnel.

\section{Appendix:}

- Pulmonary function test: this test includes recordings of your lung volumes, airflow and airways pressure by using standard equipment while you are sitting in your personal wheelchair. You will be asked to inhale and exhale in the testing devices when your nose is closed with plastic clip. This test will last about one hour.

- Respiratory Motor Control Assessment: We will ask you to repeat the pulmonary function test when you are sitting in a special hospital bed and lying on your back while we are recording the electrical impulses from your neck, chest, arms, legs, abdomen and back by placing adhesive sensors over your muscles and heart. These impulses supply us with information about how your muscles are contracting. We will also record how your chest and abdomen are moving by placing 
UofL Institutional Review Boards

IRB NUMBER: 11.0043

IRB APPROVAL DATE: 10/14/2015

IRB EXPIRATION DATE: 03/16/2016

Respiratory motor control and blood pressure regulation after spinal cord injury

Alexander Ovechkin, $\mathrm{PhD}$ elastic belts around your chest and abdomen. We will also record your blood pressure using a finger cuff. This test will last about three hours.

- Orthostatic Stress Test: Using the same methods, we will be measuring your blood pressure, heart rate and your chest and abdomen movements while lying down on your back and when the position is suddenly changed to sitting. During this test, licensed personnel will take the blood samples (approximately 4 tablespoons) from a small catheter inserted in your vein in the arm. This test will last about two hours.

- Ultrasound measurements of the heart: We will be measuring your heart and your blood vessel structure, and muscle properties using ultrasound and light technology. Continuous heart rate and blood pressure will be monitored during the test. The ultrasound will be performed while you are lying down on your side, and again while you are in a seated position. The test will last about one hour. The test will be performed by a trained and licensed Sonographer on the $11^{\text {th }}$ Floor of Frazier Rehab. The purpose of the exam is NOT to assess overall cardiovascular health or to diagnose unknown diseases. However, if abnormalities are inadvertently observed that may put your health at risk, we will report these findings to our study physician. In addition, the University of Louisville and Frazier Rehab Research Team(s) will not be held liable for undiscovered cardiovascular health issues present at the time of the exam. This test will last approximately one hour.

- Dynamic Magnetic Resonance Imaging (MRI) Scan: This test will utilize MRI to visualize the movement of your diaphragm during breathing, coughing, and swallowing. You will be lying down on a table during the test. We will place an elastic belt around your chest that measures the movement of your body wall during respiration. We will communicate to you via a headset during the test for instructions. We will ask you perform various bouts of coughing and swallowing under a predetermined set of conditions. We will supply you with a piece of hard candy to facilitate swallowing during that part of the test. Also, water will be on hand should you need it This test will last 30-40 minutes. You may be asked to sign a separate consent at the MRI facility.

- Olfactory Testing: This test will be used to assess your ability to identify different odors. We will utilize the most widely used tool available, called the UPSIT (University of Pennsylvania Smell Identification Test). We will test how accurately you can identify 40 different odors in a "scratch and sniff" format. The odorants are embedded in small strips on the pages of a booklet. There is one odorant per page. There will be a multiple choice question with four responses for each odorant. You will select the answer the corresponds to the odor you smell. We may also include a related test, called the PIT (Picture Identification Test). A trained technician will assist you with these procedures.

- Coughing/Swallowing Assessments: During this test a nose clip will be placed on your nose and you will be asked to cough forcefully through a mouthpiece that will be placed inside your mouth, into a device that measures changes in airflow. This will be repeated 2 times. This test could also be done with a small amount of water in your mouth, to test for swallowing ability. You may also be asked to swallow a small amount of water alone, for comparison.

Consent version date 09/30/15

Page 3 of 12 
UofL Institutional Review Boards IRB NUMBER: 11.0043

Alexander Ovechkin, $\mathrm{PhD}$

- Fiberoptic Endoscopic Examination of Voice: This examination involves a certified Speech Pathologist or Physician passing a small flexible endoscope tube through your nose and down into your larynx to view your vocal cords. An anesthetic spray may be applied to your nose and/or throat to avoid discomfort. Then you will be asked to perform various speech tasks while the vocal cords and folds are in view. You may also be asked to swallow a small amount of liquid during this exam to assess swallowing function.

- Voice Reading Passages: A microphone will be placed 3 inches in front of your mouth and with a comfortable voice, you will be asked to read two short passages.

- Questionnaires: You will be asked to complete three standard questionnaires. The "EAT 10" questionnaire is used to measure your degree of swallowing difficulty. The "Voice-Related Quality of Life Measure" is used to assess your degree of voicing difficulty. The "Dyspnea Index" is used to assess your degree of breathlessness and its affect on the activities of daily life.

\section{Potential Risks}

The study may involve the following physical risks and/or discomforts:

- dizziness during exercise, training and pulmonary tests $(80 \%$ chance)

- skin irritation from electrode placements $(60 \%$ chance)

- skin irritation from the vein catheterization (30\% chance)

- big changes in blood pressure and heart rate $(80 \%$ chance)

- shortness of breath (30\% chance)

- muscle and joint aches $(20 \%$ chance)

- bone fractures (1\% chance)

- Nasal inflammation or abrasion during scope insertion (less than $5 \%$ chance).

- Possible sensation of gagging during scope insertion (less than $5 \%$ chance).

If ongoing swallowing problems are present, mild coughing or choking may occur (less than $5 \%$ chance).In addition, you may suffer harms that we have not seen before. This study may involve risks that are currently unforeseeable.

\section{Possible Pregnancy Risks}

Pregnant women are excluded from this study, as the risk of training and recordings to the fetus is unknown. You should discuss these risks with your doctor before signing this consent form. Talk to your doctor about the best method of birth control to use while you are in this study. If you are pregnant or become pregnant, your unborn child may suffer harms that we have not seen before. It is important that you tell Dr. Ovechkin or someone on the research team at (502) 581-8675 right away if you become pregnant during the course of this study. If you become pregnant, your participation in this study will be terminated by the study doctor. 
UofL Institutional Review Boards Benefits

The information collected may not benefit you directly; however, the information learned in this study may be helpful to others.

\section{Alternatives}

You may choose not to participate in this research study. Choosing not to take part will not affect any treatment you are receiving.

\section{Research Related Injury}

If you are injured by being in this research study, the study doctor will arrange for you to get medical treatment. The study site, or your study doctor has not set aside money to pay for treatment of any injury. You and your insurance will be billed for the treatment of these injuries. Before you agree to take part in this research study you should find out whether your insurance will cover an injury in this kind of research. You should talk to the study doctor or staff about this. If you are injured, there is no money set aside for lost wages, discomfort, disability, etc. You do not give up your legal rights by signing this form. If you think you have a research related injury, please call your study doctor at the Frazier Rehab Institute Spinal Cord Injury Clinic at (502) 584-3376.

\section{Compensation}

You will be compensated for the travel to and from Frazier Rehab Institute for participation in recording and training sessions based on rate of $\$ 30.00$ per travel. Free parking at Frazier parking garage on the day of testing or training will be provided.

Because you will be paid to be in this study the University of Louisville must collect your name, address, social security number, ask you to sign a W-9 form, and keep records of how much you are paid. You may or may not be sent a Form 1099 by the University. This will only happen if you are paid more than $\$ 600$ in one year by the University. We are required by the Internal Revenue Service to collect this information and you may need to report the payment as income on your taxes.

This information will be protected and kept secure in the same way that we protect your other private information. If you do not agree to give us this information, we can't pay you for being in this study. You can still be in the study even if you don't want to be paid.

\section{Costs}

There will be no additional costs to you for participating. However, you or your insurance company will be billed for all office visits and procedures that are part of routine medical care. It is your responsibility to find out what costs, if any, your insurance company will cover before taking part in the study. If you are injured by the research, there may be additional cost for participating in the research. Otherwise there will be no additional cost to you. 
The Health Insurance Portability and Accountability Act of 1996 (HIPAA) provides federal safeguards for protected health information (PHI). Examples of $\mathrm{PHI}$ are your name, address, and birth date. $\mathrm{PHI}$ may also include your medical history, results of health exams and lab tests, drugs taken and results of this research study. Your PHI cannot be used or shared without your agreement, unless it meets one of the HIPAA exceptions.

State and federal privacy laws protect your health information. In most cases, health information that identifies you can be used or shared by the research team only if you give your permission by signing this form.

If you sign this form your health information will be used and shared to answer the research questions described above and to make sure that the research was done correctly. The time period when information can be used or shared ends when all activities related to this study are completed.

Your access to your health information will not be limited during this study.

You do not have to sign this form. If you do not sign this form you may not participate in the study and health information that identifies you will not be shared with the research team.

\section{Site(s) where health information about you will be used or shared for this research:}

In our research, the research team will look at and may share information about you and your health. Federal law requires that health care providers and researchers protect the privacy and security of health information that identifies you. We may ask for your health information from the following:

\section{Affiliated Sites:}

University of Louisville

Frazier Rehab Institute (Kentucky One Health)

\section{Protected health information (PHI) that will be used or shared for research}

- Complete past medical history

- Records of physical exams/tests

- Laboratory, x-ray, MRI, and other test results

- Records about your past/current medications or drugs

\section{Revocation of Research Authorization}

You may cancel the permission you have given to use and share your protected health information at any time. This means you can tell us to stop using and sharing your protected health information. If you cancel your permission:

- We will stop collecting information about you.

Consent version date 09/30/15 
- You may not withdraw information that we had before you told us to stop.

- We may already have used it or shared it.

- We may need it to complete the research.

- Staff may ask your permission to follow-up with you if there is a medical reason to do so.

To cancel your permission, you will be requested to complete a written "Revocation of Research Authorization" form located at the end of this document. You may also obtain a copy from your study doctor, designated personnel or from the Human Subjects Protections Program Office website (http://louisville.edu/research/humansubjects/links-to-forms).

\section{Confidentiality}

Total privacy cannot be guaranteed. We will protect your privacy to the extent permitted by law. If the results from this study are published, your name will not be made public. The following may look at your research and medical records:

- The funding agency (National Institutes of Health) and others hired by this agency to oversee the research

- The University of Louisville Institutional Review Board, Human Subjects Protection Program Office, Privacy Office and others involved in research administration at the University

- People who are responsible for research and HIPAA oversight at the institutions where the research is conducted

- Government agencies, such as:

- Office for Human Research Protections,

- Office of Civil Rights

- People responsible for billing, sending and receiving payments related to your participation in the study.

A description of this clinical trial will be available on http://www.ClinicalTrials.gov, as required by U.S. Law. This Web site will not include information that can identify you. At most, the Web site will include a summary of the results. You can search this Web site at any time.

\section{Security}

Your data will be kept private by assigning you a coded identification number. All research related files will be kept in a locked cabinet. The only people who will know that you are a research subject are members of the research team and, if appropriate your physicians and nurses. No information about you, or provided by you during the research, will be disclosed to others without your written permission, except if necessary to protect your rights or welfare (for example, if you are injured and need emergency care), or if required by law.

When the results of the research are published or discussed in conferences, no information will be included that will reveal your identity.

The video recorded during the training sessions will be used for teaching and research purposes only. Such teaching and research purposes can include lectures, presentations, conferences, seminars, critiques of experimental procedures, and data analysis. Your identity will not be disclosed, as neither 
Respiratory motor control and blood pressure regulation after spinal cord injury

Alexander Ovechkin, $\mathrm{PhD}$ your name nor face will be shown. You agree that the tapes shall be kept for these purposes for an indefinite time.

\section{Voluntary Participation}

Taking part in this study is completely voluntary. You may choose not to take part at all. If you decide not to be in this study, you won't be penalized or lose any benefits for which you qualify. If you decide to be in this study, you may change your mind and stop taking part at any time. If you decide to stop taking part, you won't be penalized or lose any benefits for which you qualify.

You will be told about any new information learned during the study that could affect your decision to continue in the study.

\section{Termination}

Your study doctor has the right to stop this study at any point. Your study doctor may take you out of this study with or without your okay. Reasons why this may occur include circumstances that arise which warrant doing so. The decision may be made either to protect your health and safety, or because it is part of the research plan that people who develop certain conditions may not continue to participate. If the study doctor believes that the pain or discomfort might pose a risk to you, you will be terminated from the study. If you become pregnant you will be terminated from this study.

\section{Participation in Other Research Studies}

You may not take part in this study if you are currently in another research study that will interfere with this study. It is important to let Dr. Ovechkin know if you are in another research study.

\section{Contact Persons}

If you have any questions, concerns, or complaints about the research study, please contact $\mathrm{Dr}$. Ovechkin at (502) 581-8675 or other research team members at (502) 581-8695. You will be also provided numbers to the research staff once enrolled in the study (e.g. personal cell phone numbers).

\section{Research Subject's Rights}

If you have any questions about your rights as a research subject, you may call the Human Subjects Protection Program Office at (502) 852-5188. You can discuss any questions about your rights as a research subject, in private, with a member of the Institutional Review Board (IRB). You may also call this number if you have other questions about the research, and you cannot reach the study doctor, or want to talk to someone else. The IRB is an independent committee made up of people from the University community, staff of the institutions, as well as people from the community not connected with these institutions. The IRB has reviewed this research study. 
UofL Institutional Review Boards

IRB NUMBER: 11.0043

IRB APPROVAL DATE: 10/14/2015

IRB EXPIRATION DATE: 03/16/2016

Respiratory motor control and blood pressure regulation after spinal cord injury

Alexander Ovechkin, PhD

Concerns and Complaints

If you have concerns or complaints about the research or research staff and you do not wish to give your name, you may call 1-877-852-1167. This is a 24 hour hot line answered by people who do not work at the University of Louisville. 


\section{Acknowledgement and Signatures}

This informed consent document is not a contract. This document tells you what will happen during the study if you choose to take part. Your signature indicates that this study has been explained to you, that your questions have been answered, and that you agree to take part in the study. You are not giving up any legal rights by signing this informed consent document. You will be given a signed copy of this consent form to keep for your records.

\begin{tabular}{ll}
\hline Printed Name of Subject & Signature of Subject
\end{tabular}

\begin{tabular}{|c|c|}
\hline Printed Name of & Signature of Legal Representative \\
\hline
\end{tabular}

Relationship to Subject

\begin{tabular}{lll}
\hline Printed Name of Person & Signature of Person Explaining & Date Signed \\
Explaining Consent Form & Consent Form (if other than the Investigator) &
\end{tabular}

\begin{tabular}{lll}
\hline Printed Name of Investigator & Signature of Investigator & Date Signed
\end{tabular}

\section{LIST OF INVESTIGATORS PHONE NUMBERS}

$\begin{array}{lc}\text { Alexander Ovechkin, PhD } & (502) 581-8675 \\ \text { Darryl Kaelin, MD } & (502) 407-3273 \\ \text { Sarah Wagers, MD } & (502) 852-7465 \\ \text { Megan Nelson, MD } & (502) 584-3377 \\ \text { Teresa Pitts, PhD } & (502) 852-5794 \\ \text { Swapna Chandran, MD } & (502) 583-8303 \\ \text { Fidias Eugenio Leon-Sarmiento, PhD } & (315) 510-5051\end{array}$




\section{REVOCATION OF AUTHORIZATION FOR USE AND DISCLOSURE OF YOUR HEALTH} INFORMATION FOR RESEARCH

PI Address:220 Abraham Flexner Way, $15^{\text {th }}$ Floor

PI Phone: $\underline{502-581-8675}$

Return To:

Institutional Review Board

MedCenter One, Suite 200

OR $\quad 501$ E. Broadway

Louisville, KY40202

Do not sign this letter unless you are withdrawing from this research. You will be sent confirmation that this notice was received.

To Whom It May Concern:

I would like to discontinue my participation in the research study noted above. I understand that health information already collected will continue to be used as discussed in the Authorization I signed when joining the study.

Your options are (choose one):

\section{$\square$ Withdraw from Study \& Discontinue Authorization:}

Discontinue my authorization for the future use and disclosure of protected health information. In some instances, the research team may need to use your information even after you discontinue your authorization, for example, to notify you or government agencies of any health or safety concerns that were identified as part of your study participation.

$\square$ Withdraw from Study, but Continue Authorization:

Allow the research team to continue collecting information from me and my personal health information. This would be done only as needed to support the goals of the study and would not be used for purposes other than those already described in the research authorization.

Printed Name and Signature of Subject

Signature of Subject's Legal Representative (if subject is unable to sign)

Printed Name of Subject's Legal Representative

Relationship of Legal Representative to Subject

Subject's Address

Optional:

I am ending my participation in this study because:
Date Signed

Date Signed

Birth date of Subject

Subject's Phone Number 
UofL Institutional Review Boards

IRB NUMBER: 11.0043

IRB APPROVAL DATE: 10/14/2015

IRB EXPIRATION DATE: 03/16/2016

Respiratory motor control and blood pressure regulation after spinal cord injury

Alexander Ovechkin, PhD 


\section{CURRICULUM VITA}

BONNIE LEGG DITTERLINE, M.S.

502-751-1410

Bonita.Elizabeth@icloud.com

\section{EDUCATION}

UNIVERSITY OF LOUISVILLE, PH.D. CANDIDATE AND GRADUATE FELLOW, PHYSIOLOGY AND BIOPHYSICS

- Developed a research project in order to study the relationship between cardiovascular regulation and pulmonary function in Chronic Spinal Cord Injury PRESENTATIONS

6 June, 2015, KSCHIRT Research Group, Louisville. Oral Presentation: "Cardiovascular Regulation Post Respiratory Motor Training in Persons with Chronic SCl."

21 May, 2015, KSCHIRT Research Symposium, Louisville. Oral Presentation: "Effects of Respiratory Motor Training on Cardiovascular Function in Persons with Chronic SCI."

28 March, 2015, Experimental Biology Conference, Boston: "How Respiratory Muscle Training Affects Breathing in Individuals with Spinal Cord Injury." SZ Aslan, EH Brown, M Chopra, B Ditterline, G. Singh, SC Aslan, AV Ovechkin

16 May 2014, KSCHIRT Research Symposium, Lexington. Poster Presentation: "Effects of Respiratory Muscle Training on Pulmonary Function and Baroreflex Sensitivity in Persons with Chronic Spinal Cord Injury." Bonnie Ditterline, SC Aslan, AV Ovechkin.

21 April 2014, Neuroscience Day, Louisville. Poster Presentation: "Evaluation of Baroreceptor Sensitivity and Sympathetic Activity in Chronic Spinal Cord Injury." Bonnie Ditterline, SC Aslan, AV Ovechkin.

21 March 2013, Qualifying Examination, University of Louisville. "Mechanisms of Cardiovascular Impairment in Chronic Spinal Cord Injury: Role of Respiratory Motor Function." 
PAPERS

"CARDIOVASCULAR REGULATION AND EFFECTS OF RESPIRATORY MOTOR TRAINING IN PATIENTS WITH CHRONIC SPINAL CORD INJURY" Dissertation, 2015. "Baroreceptor Reflex During Forced Expiratory Maneuvers in Individuals with Chronic Spinal Cord Injury." Submitted, 2015. "Baroreceptor Sensitivity Post Respiratory Muscle Training in the Chronic Spinal Cord Injured Population." In progress, 2015.

MEMBERSHIPS

American Physiological Society (2011-Present), Society for Neuroscience (2013-Present)

UNIVERSITY OF LOUISVILLE, DECEMBER 2010, M.S. PHYSIOLOGY AND BIOPHYSICS

- Developed research project and statistical analysis to assess pulmonary function in persons with spinal cord injury.

- Maintained GPA above a 3.5 despite rigorous course load.

RESEARCH PROJECT

Worked with Kentucky Spinal Cord Injury Research Center at the University of Louisville and Frazier Rehabilitation and Neuroscience Center to design a project to evaluate the relationship between pulmonary function and spinal cord injury; assisted with IRB approval and amendment writing; gathered data and created analysis to evaluate data; assisted and gathered data for other projects run by fellow PhD candidates and Postdoctoral associates.

UNIVERSITY OF KENTUCKY, DECEMBER 2008, B.S. NUTRITION AND FOOD SCIENCE, ANTHROPOLOGY

- $100 \%$ self-supported through financing and working $30+$ hours per week.

- Graduated with 3.2 GPA and made Deans List despite full-time work- and school-load.

WORK EXPERIENCE

FRAZIER REHABILITATION AND NEUROSCIENCE CENTER, AUGUST 2011-PRESENT

GRADUATE RESEARCH FELLOW

-Developed research project to evaluate the relationship between cardiovascular regulation and pulmonary function in the spinal cord injured population 
- Helped design and create protocol in order to accurately study cardiovascular regulation and baroreceptor activity based on existing literature

-Developed novel methods and created statistical analyses to assess this relationship

-Worked with my team to gather experimental data and train participants between experiments

-Assisted with IRB submissions and amendments to remain up to date with all related project protocols and ensure we remain HIPAA compliant

-Assisted with recruiting and consenting participants for my project - Performed cardiovascular assessments and experiments for other projects within the Neuroscience Collaborative Center, including clinical trials and other R01 research projects

\section{Galen College of Nursing, August 2015-Present}

ADJUNCT INSTRUCTOR, ANATOMY AND PHYSIOLOGY II

-Developed materials to teach students in Anatomy and Physiology Theory and Lab Classes, including exams, assignments, and lesson plans

-Served students from broad socioeconomic and education backgrounds to help them attain their goals of graduating with a Nursing Degree, including private tutoring, and remediation -Assisted the faculty with curricula development to serve the school's broader goal of BSN accreditation

NORTON CANCER INSTITUTE, JANUARY 2011-JULY 2011 CLINICAL RESEARCH ASSISTANT

- Assisted with existing Breast, Colon and Cervical cancer research projects, including gathering and entering data as well as communicating with participants enrolled in the research projects

- Analyzed data for projects presented locally and regionally, as well as editing and proofreading documents prior to submission or presentation

- Worked to develop future research projects and directions, from creating and implementing ideas to gathering necessary instruments.

- Kept updated with research relevant to current projects as well as research relevant to future projects. 


\section{FRAZIER REHABILITATION AND NEUROSCIENCE CENTER, JANUARY}

2010-DECEMBER 2010

\section{GRADUATE RESEARCH ASSISTANT}

- Created and worked on my own research project with my mentor's guidance

- Assisted with experiments from a variety of research projects

- Edited and proofread documents prior to submission while maintaining deadlines 